PREPARED FOR SUBMISSION TO JHEP

\title{
Relative Entropy and Holography
}

\author{
David D. Blanco ${ }^{a}$ Horacio Casini $^{a}$ Ling-Yan Hung $^{b}$ Robert C. Myers ${ }^{c}$ \\ ${ }^{a}$ Centro Atómico Bariloche, 8400-S.C. de Bariloche, Río Negro, Argentina \\ ${ }^{b}$ Department of Physics, Harvard University, Cambridge, MA 02138 USA \\ ${ }^{c}$ Perimeter Institute for Theoretical Physics, Waterloo, Ontario N2L 2Y5, Canada \\ E-mail: blancod@ib.cnea.gov.ar, casini@cab.cnea.gov.ar, \\ lhung@physics.harvard.edu, rmyers@perimeterinstitute.ca
}

\begin{abstract}
Relative entropy between two states in the same Hilbert space is a fundamental statistical measure of the distance between these states. Relative entropy is always positive and increasing with the system size. Interestingly, for two states which are infinitesimally different to each other, vanishing of relative entropy gives a powerful equation $\Delta S=\Delta H$ for the first order variation of the entanglement entropy $\Delta S$ and the expectation value of the modular Hamiltonian $\Delta H$. We evaluate relative entropy between the vacuum and other states for spherical regions in the AdS/CFT framework. We check that the relevant equations and inequalities hold for a large class of states, giving a strong support to the holographic entropy formula. We elaborate on potential uses of the equation $\Delta S=\Delta H$ for vacuum state tomography and obtain modified versions of the Bekenstein bound.
\end{abstract}

ARXIV EPRINT: 1305.3182 


\section{Contents}

1 Introduction 1

2 Simple examples testing holographic entanglement entropy $\quad 6$

2.1 Boosted black brane 9

2.2 Charged black brane 11

3 General analysis $\quad 13$

3.1 Linear corrections to relative entropy $\quad 15$

3.2 Quadratic corrections to relative entropy 20

3.3 Corrections from additional operators 24

3.4 Corrections for general entangling surfaces 29

4 Two-dimensional boundary theories 32

4.1 Thermal Rindler space 36

5 Puzzles about localization $\quad 39$

5.1 Complementary regions in a pure state 40

5.2 An inequality for $\Delta\langle H\rangle \quad 42$

5.3 Annular regions 43

6 Discussion $\quad 45$

A Comments on Relative Entropy $\quad 53$

A.1 $\Delta S=\Delta\langle H\rangle$ for first order perturbations $\quad 54$

A.2 Strong subadditivity 56

A.3 Second law of thermodynamics $\quad 56$

$\begin{array}{lll}\text { A.4 Bekenstein bound } & 58\end{array}$

\section{Introduction}

Entanglement entropy has emerged as a remarkable theoretical tool providing new insights into a variety of topics in physics. For example, in condensed matter theory, it can be used to distinguish new topological phases or different critical points $[1,2]$. In the context of quantum field theory (QFT), entanglement entropy has been proposed 
as a useful probe of phase transitions in gauge theories [3]. Further, it has provided new insights on the structure of renormalization group flows $[4,5]$. In particular, it was instrumental in establishing new c-theorems in three and higher dimensions [6, 7]. Of course, entanglement entropy has long been proposed as the origin of black hole entropy [8-10]. More recently, considerations of entanglement have led to an exciting new discussion on the nature of Hawking radiation and black hole evaporation [11-13]. At a more fundamental level, it has been suggested that entanglement entropy may play an important role in understanding the quantum structure of spacetime, e.g., [14-16].

Entanglement entropy has also figured in many recent discussions of gauge/gravity duality. The entanglement entropy in the boundary QFT is determined with an elegant geometric calculation in the dual gravity theory [17]. In particular, the entanglement entropy between a (spatial) region $V$ and its complement $\bar{V}$ in the boundary is computed by

$$
S(V)=\frac{2 \pi}{\ell_{\mathrm{P}}^{d-1}} \operatorname{ext}_{v \sim V}^{\operatorname{ext}}[A(v)]
$$

where one extremizes over all surfaces $v$ in the bulk spacetime which are homologous to the boundary region $V$. Here, we have adopted the convention $\ell_{\mathrm{P}}^{d-1}=8 \pi G_{\mathrm{N}}$ where $d$ is the spacetime dimension of the boundary. This prescription (1.1) was found to pass wide range of consistency tests, e.g., see [17-19]. However, a derivation was provided for the special case of a spherical entangling surface in [20] and quite remarkably, [21] recently extended this derivation to general (smooth) entangling surfaces.

Quantum information theory provides a variety of other tools with which we might refine our understanding of entanglement in holographic theories. For example, Rényi entropies are an infinite family of measures of entanglement [22, 23], which in principle provide a full description of the density matrix spectrum, e.g., [24]. Unfortunately, progress towards understanding holographic Rényi entropies has been more limited [18, 25, 26]. In particular, a good understanding of Rényi entropies has been developed for a two-dimensional boundary CFT and further, these quantities are easily computed for a spherical entangling surface in any number of dimensions [26]. However, an effective and efficient approach to calculate holographic Rényi entropy for more general situations is still lacking.

In the present paper, we will consider another quantity known as the relative entropy in the context of holography. The relative entropy between two states in the same Hilbert space yields a fundamental statistical measure of the distance between these states. Given two density matrices $\rho_{1}$ and $\rho_{0}$, the relative entropy $S\left(\rho_{1} \mid \rho_{0}\right)$ is defined as

$$
S\left(\rho_{1} \mid \rho_{0}\right)=\operatorname{tr}\left(\rho_{1} \log \rho_{1}\right)-\operatorname{tr}\left(\rho_{1} \log \rho_{0}\right)
$$


In general, $S\left(\rho_{1} \mid \rho_{0}\right) \geq 0$ where it vanishes if and only if the states are equal. Further, if $\rho_{1}$ and $\rho_{0}$ describe reduced states on some region $V$, the relative entropy always increases with the size of $V$, i.e., $S\left(\rho_{1} \mid \rho_{0}\right)$ increases under inclusion (for a review see, e.g., $[27,28])$. When the set is small, both states should approach the vacuum state on the operator algebra of the region, and then the relative entropy tends to zero.

The positivity of $S\left(\rho_{1} \mid \rho_{0}\right)$ can be given a physical interpretation in terms of thermodynamics. If the state $\rho_{0}$ is thermal with respect to the Hamiltonian $H$, i.e., $\rho_{0}=\frac{e^{-H / T}}{\operatorname{tr}\left(e^{-H / T}\right)}$, then the relative entropy with any other state $\rho_{1}$ can be expressed as

$$
S\left(\rho_{1} \mid \rho_{0}\right)=\frac{1}{T}\left(F\left(\rho_{1}\right)-F\left(\rho_{0}\right)\right),
$$

where $F(\rho)$ is the free energy given by

$$
F(\rho)=\operatorname{tr}(\rho H)-T S(\rho) .
$$

We emphasize that $\rho_{1}$ can be any other state and need not be thermal. Hence the temperature used to define $F\left(\rho_{1}\right)$ is that of the initial state $\rho_{0}$. Now given the expression in eq (1.3), the positivity of the relative entropy is equivalent to the fact that the free energy at a fixed temperature $T$ is minimized by the thermal equilibrium state.

Now consider the reduced density matrices describing states of a QFT on a region $V$. Since any such density matrix is both Hermitian and positive semidefinite, it can be expressed as

$$
\rho=\frac{e^{-H}}{\operatorname{tr}\left(e^{-H}\right)}
$$

for some Hermitian operator $H$. The latter is known as the modular Hamiltonian in the literature on axiomatic quantum field theory, e.g., $[29],{ }^{1}$ while it is referred to as the entanglement Hamiltonian in the condensed matter theory literature, e.g., [30]. The denominator is included in the above expression to ensure the normalization $\operatorname{tr}(\rho)=1$ and it could instead be absorbed with an additive constant in $H$. However, it will be convenient to maintain this form below. While $H$ plays an important role in addressing certain questions, we emphasize that generically the modular Hamiltonian is not a local operator and the evolution generated by $H$ would not correspond to a local (geometric) flow.

Returning to our considerations of the relative entropy and given eq. (1.5), formally we can say the state $\rho_{0}$ is thermal with a temperature $T=1$. Hence we can apply eq. (1.3) to express the relative entropy as

$$
S\left(\rho_{1} \mid \rho_{0}\right)=\Delta\langle H\rangle-\Delta S
$$

\footnotetext{
${ }^{1}$ The precise definition of the modular Hamiltonian for a region $V$ in algebraic QFT also includes an extension of $H$ in eq. (1.5) to the algebra of operators outside the region $V$.
} 
where

$$
\Delta\langle H\rangle=\operatorname{tr}\left(\rho_{1} H\right)-\operatorname{tr}\left(\rho_{0} H\right) \quad \text { and } \quad \Delta S=S\left(\rho_{1}\right)-S\left(\rho_{0}\right) .
$$

Now the positivity of the relative entropy requires ${ }^{2}$

$$
\Delta\langle H\rangle \geq \Delta S
$$

That is, in comparing two states, the variation of the entanglement entropy is bounded by the variation of the expectation value of the modular Hamiltonian. Much of our analysis in this paper will focus on examining this inequality in a holographic setting. The holographic prescription (1.1) allows us to calculate the necessary entanglement entropies and hence $\Delta S$. Further if the modular Hamiltonian is known, we can also evaluate $\Delta\langle H\rangle$, e.g., after evaluating the expectation value of the stress energy $\left\langle T_{a b}\right\rangle$ using standard methods. Unfortunately there are only few simple cases where the modular Hamiltonian is explicitly known, as we describe below.

The cases where the precise form of $H$ is known correspond to special situations, in which the modular Hamiltonian (and the corresponding internal time flow generated by $H$ ) are local. ${ }^{3}$ Let us enumerate a few of these cases here: One well-known example is given by the vacuum state in any QFT restricted to the half space $x>0$. In this case, the modular Hamiltonian is proportional to $K$, the boost generator in the $x$ direction $[31]$,

$$
H=2 \pi K=2 \pi \int_{x>0} d^{d-1} x x T_{00}(\vec{x}) .
$$

In this case, $H$ generates a geometric flow along the boost orbits in the Rindler wedge. Of course, the density matrix then has a thermal interpretation with respect to time translations along these orbits [32]. A second example corresponds to the vacuum of a conformal field theory and a spherical entangling surface, which yields

$$
H=2 \pi \int_{|x|<R} d^{d-1} x \frac{R^{2}-r^{2}}{2 R} T_{00}(\vec{x}) .
$$

This result is easily derived from eq. (1.9) since there is a special conformal transformation (and translation) which maps the Rindler wedge to the causal development of the ball $|x|<R$ - e.g., see [29, 33]. Another situation where the modular Hamiltonian

\footnotetext{
${ }^{2}$ This inequality can be regarded as a generalized statement of the Bekenstein bound which holds for any region in QFT. This is explained in more detail in the appendix A.4.

${ }^{3}$ The simplest example is given by considering a global thermal state, with temperature $T$, and taking $V$ to be the whole space. Then, the modular Hamiltonian is simply the ordinary (local) Hamiltonian divided by $T$, as is evident from eq. (1.5), and so $H$ simply generates ordinary time translations.
} 
is known to be local is the case of a two-dimensional CFT in a thermal state (with temperature $T$ ) on the Rindler wedge [34]. In this case, the modular Hamiltonian can be expressed as

$$
H=\frac{1}{T} \int_{x>0} d x\left(1-e^{-2 \pi T x}\right) T_{00}(\vec{x}) .
$$

In the following, we focus primarily on the case of a spherical entangling surface with $H$ given by eq. (1.10). As described above, our strategy will be to use holographic techniques to calculate both $\Delta\langle H\rangle$ and $\Delta S$ and to test whether the inequality (1.8) is satisfied. We will find that eq. (1.8) is always satisfied but further, that in many of our examples, the inequality is in fact saturated to linear order in the perturbations of the state. The appearance of an equality in these cases can be understood because we are examining the relative entropy of two nearby states. Consider choosing a fixed reference state $\rho_{0}$ and then moving through a family of states $\rho_{1}(\lambda)$ with a parameter $\lambda$ such that $\rho_{1}(\lambda=0)=\rho_{0}$. Since the two states coincide for $\lambda=0$, we have that $S\left(\rho_{1}(0) \mid \rho_{0}\right)=0$ but $S\left(\rho_{1}(\lambda) \mid \rho_{0}\right)>0$ for both positive and negative $\lambda$. Therefore if $S\left(\rho_{1}(\lambda) \mid \rho_{0}\right)$ is a smooth function of $\lambda$, its first derivative must vanish at $\lambda=0$. Of course, this vanishing implies

$$
\Delta\langle H\rangle=\Delta S
$$

to first order in $\lambda$ (at $\lambda=0)$ - see further discussion in the appendix A. In thermodynamical terms of eq. (1.3) this is the well known equation $d E=T d S$ holding for nearby equilibrium states.

While the above approach tests the positivity of the relative entropy, we can also use our holographic results to examine the monotonicity constraint mentioned below eq. (1.2). That is, the relative entropy should increase as the radius of the spherical entangling surface increases. Of course, this property can only be tested in the cases where $\Delta\langle H\rangle \neq \Delta S$, where we should find

$$
\partial_{R} S\left(\rho_{1} \mid \rho_{0}\right)=\partial_{R}[\Delta\langle H\rangle-\Delta S] \geq 0
$$

The remainder of the paper is organized as follows: In section 2, we test relative entropy bounds and the linear equality (1.12) for simple examples containing black branes in the bulk. In section 3 we analyze general linear perturbations of the vacuum finding agreement with eq. (1.12). We also compute quadratic perturbations and find in all our examples that relative entropy is positive and increasing. In section 4 we analyze some examples in $d=2$ which allow for exact analytic calculations of the entropy. We discuss some puzzles about localizations of contributions to $\Delta\langle H\rangle$ in section 5 . We conclude with a summary of the results and further comments on section 6 . In particular, we discuss the potential of eq. (1.12) to make vacuum state tomography 
using entanglement entropy, and argue the results of section 3 are powerful enough to reconstruct the full density matrix in a sphere from the minimal area prescription for the entropy, in perfect accord with the CFT result. Finally, in appendix A we review several issues related to relative entropy, including its relation to the strong subadditivity property of entanglement entropy, the second law of thermodynamics, and the Bekenstein bound.

\section{Simple examples testing holographic entanglement entropy}

As commented above, our strategy will be to test the inequality (1.8) in a holographic setting for the case of a spherical entangling surface, for which the modular Hamiltonian (1.10) is known. The RT prescription [17] allows us to calculate the entanglement entropies and hence $\Delta S$. But in these cases, we can also evaluate $\Delta\langle H\rangle$ given the expectation value of the stress energy $\left\langle T_{a b}\right\rangle$. In this section, while our reference state (defining $\rho_{0}$ ) is the vacuum of the CFT, our second state (defining $\rho_{1}$ ) will be the holographic dual of a black hole. This is a warm-up exercise to give us some insight before proceeding with a more general analysis in the next section.

The bulk solution dual to the vacuum of the $d$-dimensional boundary CFT is simply empty $\mathrm{AdS}_{d+1}$ space, which we write in the Poincaré coordinates:

$$
d s^{2}=\frac{L^{2}}{z^{2}}\left(-d t^{2}+d \vec{x}_{d-1}^{2}+d z^{2}\right) .
$$

Now we are considering a spherical entangling surface in the boundary theory, i.e., the region $V$ is the ball $\{t=0, r \leq R\}$. Now the stress tensor has vanishing expectation value in the vacuum state and so the expectation value of the modular Hamiltonian (1.10) vanishes for this state, i.e., $\langle H\rangle_{0}=\operatorname{tr}\left(\rho_{0} H\right)=0$. Applying the holographic prescription (1.1) to evaluate the entanglement entropy, one finds that the minimal area surface $v$ is given by [17]

$$
z=z_{0}(r) \equiv \sqrt{R^{2}-r^{2}}
$$

Hence the entanglement entropy takes the form

$$
S_{0}=2 \pi \frac{A(v)}{\ell_{\mathrm{P}}^{d-1}}=2 \pi \frac{L^{d-1}}{\ell_{\mathrm{P}}^{d-1}} \Omega_{d-2} \int_{0}^{R} d r \frac{r^{d-2}}{z^{d-1}} \sqrt{1+\partial_{r} z^{2}},
$$

where $\Omega_{d-2}$ denotes the area of a unit $(d-2)$-sphere, i.e.,

$$
\Omega_{d-2}=\frac{2 \pi^{(d-1) / 2}}{\Gamma((d-1) / 2)}
$$


We will not need to explicitly evaluate eq. (2.3) for the following, however, the interested reader may find the result in $[17,20]$.

For our second state defining $\rho_{1}$, we take the holographic dual of a bulk black brane solution, i.e., a planar AdS black hole. In general, the (expectation value of the) stress tensor dual to a stationary black brane takes the form of that for an ideal fluid,

$$
\left\langle T_{\mu \nu}\right\rangle=(\varepsilon+P) u_{\mu} u_{\nu}+P \eta_{\mu \nu},
$$

where $\varepsilon, P$ and $u_{\mu}$ correspond to the energy density, pressure and $d$-velocity of the fluid, respectively. Since the boundary theory is a CFT, we also have $\left\langle T^{\mu}{ }_{\mu}\right\rangle=0$ which imposes $P=\varepsilon /(d-1)$.

As our first example, we consider a static planar AdS black hole, for which the metric may be written as

$$
d s^{2}=\frac{L^{2}}{z^{2}}\left(-f(z) d t^{2}+d \vec{x}_{d-1}^{2}+\frac{d z^{2}}{f(z)}\right) \text { with } f(z)=1-\frac{z^{d}}{z_{\mathrm{h}}^{d}} .
$$

In this case, the dual plasma is at rest, i.e., $u^{\mu}=\left(1, \overrightarrow{0}_{d-1}\right)$, and so eq. (2.5) reduces to

$$
\left\langle T_{\mu \nu}\right\rangle=\varepsilon \operatorname{diag}(1,1 /(d-1), 1 /(d-1), \cdots) .
$$

Now the usual holographic dictionary $[35,36]$ gives the energy density as

$$
\varepsilon=\frac{d-1}{2} \frac{L^{d-1}}{\ell_{\mathrm{P}}^{d-1}} \frac{1}{z_{\mathrm{h}}^{d}} .
$$

The latter can be interpreted as $\varepsilon=c T^{d}$ using the expression for the black hole temperature:

$$
T=\frac{d}{4 \pi z_{\mathrm{h}}}
$$

With these expressions, it is straightforward to evaluate the expectation of the modular Hamiltonian (1.10) for this state,

$$
\begin{aligned}
\langle H\rangle_{1} & =\pi \Omega_{d-2} \frac{\varepsilon}{R} \int_{0}^{R} d r r^{d-2}\left(R^{2}-r^{2}\right) \\
& =\frac{2 \pi \Omega_{d-2}}{d^{2}-1} R^{d} \varepsilon .
\end{aligned}
$$

Hence we arrive at

$$
\Delta\langle H\rangle=\langle H\rangle_{1}-\langle H\rangle_{0}=\frac{\pi \Omega_{d-2}}{d+1} \frac{L^{d-1}}{\ell_{\mathrm{P}}^{d-1}} \frac{R^{d}}{z_{\mathrm{h}}^{d}}
$$


after replacing $\varepsilon$ using eq. (2.8).

Now to complete our comparison in eq. (1.8), we need to evaluate the entanglement entropy for a spherical entangling surface in the black brane background. Applying the holographic prescription (1.1), the entropy functional in this new background becomes

$$
S_{1}=2 \pi \frac{L^{d-1}}{\ell_{\mathrm{P}}^{d-1}} \Omega_{d-2} \int_{0}^{R} d r \frac{r^{d-2}}{z^{d-1}} \sqrt{1+\frac{\left(\partial_{r} z\right)^{2}}{f(z)}},
$$

where $f(z)$ is the metric function given in eq. (2.6). In principle, we could extremize the above expression, i.e., solve for $z(r)$, and evaluate the entropy at an arbitrary temperature, but this would require a numerical evaluation. ${ }^{4}$ To make progress analytically, we will carry out a perturbative calculation for 'small' spheres or low temperatures, in which we consider the limit $R / z_{\mathrm{h}} \ll 1$ (or alternatively, $R T \ll 1$ ). In this case, the minimal surface is only probing the asymptotic region of the black brane geometry (2.6) and so the solution deviates only slightly from the AdS solution (2.2), i.e., $z(r)=z_{0}(r)+\delta z(r)$. Now since $z_{0}(r)$ extremizes the entropy functional for the AdS background in eq. (2.3), the deviation $\delta z(r)$ will not modify the result at first order in our perturbative calculation. ${ }^{5}$ Hence, the leading order change in the entropy comes from evaluating eq. (2.12) with $z=z_{0}(r)$ and determining the leading contribution in $R / z_{\mathrm{h}}$. Expanding eq. (2.12) to leading order in $1 / z_{\mathrm{h}}^{d}$ yields

$$
\begin{aligned}
\Delta S & =\left.\pi \frac{L^{d-1}}{\ell_{\mathrm{P}}^{d-1}} \Omega_{d-2} \int_{0}^{R} d r \frac{r^{d-2} z\left(\partial_{r} z\right)^{2}}{z_{\mathrm{h}}^{d} \sqrt{1+\left(\partial_{r} z\right)^{2}}}\right|_{z=z_{0}(r)}=\pi \frac{L^{d-1}}{\ell_{\mathrm{P}}^{d-1}} \Omega_{d-2} \int_{0}^{R} d r \frac{r^{d}}{z_{\mathrm{h}}^{d} R} \\
& =\frac{\pi \Omega_{d-2}}{d+1} \frac{L^{d-1}}{\ell_{\mathrm{P}}^{d-1}} \frac{R^{d}}{z_{\mathrm{h}}^{d}}
\end{aligned}
$$

Hence comparing to eq. (2.11), we see that to leading order

$$
\Delta\langle H\rangle=\Delta S
$$

and so we have saturated the inequality in eq. (1.8)! Of course, this equality is perhaps not so surprising given the discussion around eq. (1.12). ${ }^{6}$ Here we are looking at a

\footnotetext{
${ }^{4}$ The interested reader is referred to [37] for various interesting analytic approximations.

${ }^{5}$ As well as a bulk term proportional to the equations of motion, the first order variation by $\delta z(r)$ will also generate a total derivative and so one may worry that there is a nonvanishing boundary term at the cut-off surface. However, a careful examination shows that this boundary term actually vanishes. The simplest approach is to simply define the entangling surface directly at the cut-off surface and then $\delta z$ vanishes there.

${ }^{6}$ Actually the discussion there does not apply directly to the present example since one would not consider the energy density of the fluid dual to the black hole taking negative values. However, one might consider a state where stress tensor locally takes the form in eq. (2.8) but with $\varepsilon<0$ in a small region around the entangling sphere.
} 
family of density matrices characterized by the temperature $T$ and our perturbative calculation is evaluating the leading order change in $\langle H\rangle$ and $S$, which appears linearly at order $(R T)^{d}$. Of course, it would be interesting to evaluate both sides of eq. (1.8) at next order in the perturbative expansion, but we leave this exercise to our general analysis in section 3. Of course, given the equality in eq. (2.14), we can not test the monotonicity inequality (1.13) at this order. We should add that calculations similar to those above has also been done in [38], without any reference to relative entropy.

\subsection{Boosted black brane}

We now repeat these calculations for a boosted AdS black brane. That is, the second state defining $\rho_{1}$ is a thermal plasma which is uniformly boosted in a certain direction. Hence this new state $\rho_{1}$ is characterized by the temperature $T$ and the velocity $v$. Our calculations will be to leading order in the temperature and all orders in the velocity.

The stress tensor takes the form given in eq. (2.5) now with $u^{\mu}=\left(\gamma, \gamma v, \overrightarrow{0}_{d-2}\right)$ where $\gamma=1 / \sqrt{1-v^{2}}$, as well as $P=\varepsilon /(d-1)$. In particular, we have

$$
\left\langle T_{00}\right\rangle=\varepsilon\left(1+\frac{d}{d-1} \gamma^{2} v^{2}\right) .
$$

The corresponding bulk black brane solution is simply derived by applying a boost along, say, the direction of $x^{1} \equiv x$ directly to the metric in eq. (2.6). It is convenient to write the resulting metric as

$$
d s^{2}=\frac{L^{2}}{z^{2}}\left[-d t^{2}+d x^{2}+\gamma^{2} \frac{z^{d}}{z_{\mathrm{h}}^{d}}(d t+v d x)^{2}+d \vec{x}_{d-2}^{2}+\frac{d z^{2}}{1-\frac{z^{d}}{z_{\mathrm{h}}^{d}}}\right] .
$$

With the usual holographic approach [35, 36], one can verify eq. (2.15) with $\varepsilon$ given by eq. (2.8), as before. Now we wish to evaluate the change in the (expectation value of the) modular Hamiltonian (1.10) for the boosted plasma. Since the energy density is still uniform the calculation of $\langle H\rangle_{1}$ is the same as before, up to the additional overall pre-factor in eq. (2.15). Hence, we arrive at

$$
\Delta\left\langle H^{\prime}\right\rangle=\Delta\langle H\rangle\left(1+\frac{d}{d-1} \gamma^{2} v^{2}\right)
$$

where $\Delta\langle H\rangle$ is the variation of the modular Hamiltonian given in eq. (2.11).

Now in principle, because the background (2.16) is stationary (but not static), we must apply the covariant prescription suggested by [39] to evaluate the holographic entanglement entropy. In fact, the holographic prescription presented in eq. (1.1) already 
accommodates this situation. In this new background, we would need to find the extremal surface with a profile defined by $z=z(x, y)$ and $t=t(x, y)$ where $y^{2} \equiv \sum_{i=2}^{d-1}\left(x^{i}\right)^{2}$ - in particular, note that the extremal surface will not remain on a fixed time slice in the bulk. However, our goal is to evaluate the change in the entanglement entropy $\Delta S^{\prime}$ and reasoning as in the previous section, we deduce that the leading change will be determined by simply evaluating the area in the new background geometry with the zero-temperature profile (2.2). Hence we can ignore the deviations of the extremal surface away from the constant time slice in the following.

With a profile $z=z(x, y)$, it is straightforward to show that the entropy in the boosted background (2.16) takes the form

$$
S_{1}^{\prime}=2 \pi \frac{L^{d-1}}{\ell_{\mathrm{P}}^{d-1}} \Omega_{d-3} \int_{-R}^{R} d x \int_{0}^{\sqrt{R^{2}-x^{2}}} d y \frac{y^{d-3}}{z^{d-1}}\left[\left(1+\gamma^{2} v^{2} \frac{z^{d}}{z_{\mathrm{h}}^{d}}\right)\left(1+\frac{\partial_{y} z^{2}}{f(z)}\right)+\frac{\partial_{x} z^{2}}{f(z)}\right]^{1 / 2},
$$

where again $f(z)$ is given in eq. (2.6). While no approximations were made in evaluating $\Delta\left\langle H^{\prime}\right\rangle$ in eq. (2.17), as before, in evaluating the change in the entropy, we will work to leading order in the limit $R / z_{\mathrm{h}} \ll 1$. Again, applying the same reasoning as in our previous calculations, we conclude that the leading order change in the entropy comes simply from evaluating eq. (2.18) with the zero-temperature profile (2.2), i.e., $z=z_{0}(r)=\sqrt{R^{2}-x^{2}-y^{2}}$. We first expand the above expression to leading order in $1 / z_{\mathrm{h}}^{d}$ and then subtract the zero'th order contribution (2.3), which yields

$$
\Delta S^{\prime}=\pi \frac{L^{d-1}}{\ell_{\mathrm{P}}^{d-1}} \Omega_{d-3} \int_{-R}^{R} d x \int_{0}^{\sqrt{R^{2}-x^{2}}} d y \frac{y^{d-3} z}{z_{\mathrm{h}}^{d} \sqrt{1+\partial_{r} z^{2}}}\left[\partial_{r} z^{2}+\gamma^{2} v^{2}\left(1+\partial_{y} z^{2}\right)\right],
$$

where we have simplified $\partial_{x} z^{2}+\partial_{y} z^{2}=\partial_{r} z^{2}$ in anticipation of substituting $z=z_{0}(r)$. With this substitution, the first term in the square brackets will yield precisely the 'unboosted' result $\Delta S$, given in eq. (2.13). Hence we are left with

$$
\begin{aligned}
\Delta S^{\prime} & =\Delta S+\left.\pi \frac{L^{d-1}}{\ell_{\mathrm{P}}^{d-1}} \Omega_{d-3} \gamma^{2} v^{2} \int_{-R}^{R} d x \int_{0}^{\sqrt{R^{2}-x^{2}}} d y \frac{y^{d-3} z\left(1+\partial_{y} z^{2}\right)}{z_{\mathrm{h}}^{d} \sqrt{1+\partial_{r} z^{2}}}\right|_{z=z_{0}(r)} \\
& =\Delta S+\pi \frac{L^{d-1}}{\ell_{\mathrm{P}}^{d-1}} \Omega_{d-3} \gamma^{2} v^{2} \int_{-R}^{R} d x \int_{0}^{\sqrt{R^{2}-x^{2}}} d y \frac{y^{d-3}\left(R^{2}-x^{2}\right)}{z_{\mathrm{h}}^{d} R} \\
& =\Delta S+\pi \frac{L^{d-1}}{\ell_{\mathrm{P}}^{d-1}} \Omega_{d-3} \gamma^{2} v^{2} \frac{R^{d}}{z_{\mathrm{h}}^{d}} \frac{\sqrt{\pi}}{d-2} \frac{\Gamma(d / 2+1)}{\Gamma(d / 2+3 / 2)} \\
& =\Delta S\left(1+\frac{d}{d-1} \gamma^{2} v^{2}\right),
\end{aligned}
$$

where we have used eqs. (2.4) and (2.13) to produce the simple expression in the final line. 
Recall that we found $\Delta\langle H\rangle=\Delta S$ in the previous section and hence in comparing to eqs. (2.17) and (2.20), we again find that to leading order

$$
\Delta\left\langle H^{\prime}\right\rangle=\Delta S^{\prime}
$$

for the boosted plasma. While the expressions appearing in the calculations above are somewhat more complicated, we may have still anticipated this equality from the discussion around eq. (1.12). In this case, we are considering a family of density matrices characterized by the temperature $T$ and the velocity $v$. While our calculations are valid to all orders in the velocity, we are only evaluating $\Delta\left\langle H^{\prime}\right\rangle$ and $\Delta S^{\prime}$ to leading order in $(R T)^{d}$.

\subsection{Charged black brane}

Continuing the analysis of section 2, another interesting background to consider as defining $\rho_{1}$ is a charged AdS black brane. In this case, the state in the boundary theory is characterized by the chemical potential $\mu$, as well as the temperature $T$. Our calculations will be to leading order in $R T$, however, we allow $\mu / T$ to be order one.

In this case, we consider the bulk gravity action

$$
I=\frac{1}{2 \ell_{\mathrm{P}}^{d-1}} \int d^{d+1} x \sqrt{-g}\left(\frac{d(d-1)}{L^{2}}+R-\frac{L^{2}}{4} F_{\mu \nu} F^{\mu \nu}\right)
$$

with $d \geq 3 .^{7}$ The metric for a planar charged black hole can be written as

$$
d s^{2}=\frac{L^{2}}{z^{2}}\left(-h(z) d t^{2}+d \vec{x}_{d-1}^{2}+\frac{d z^{2}}{h}\right)
$$

where

$$
h=1-\left(1+z_{\mathrm{h}}^{2} q^{2}\right) \frac{z^{d}}{z_{\mathrm{h}}^{d}}+q^{2} \frac{z^{2 d-2}}{z_{\mathrm{h}}^{2 d-4}},
$$

and the corresponding gauge potential has only a single nonvanishing component

$$
A_{0}(z)=\sqrt{\frac{2(d-1)}{d-2}} q\left(1-\frac{z^{d-2}}{z_{\mathrm{h}}^{d-2}}\right) .
$$

Here, $z=z_{\mathrm{h}}$ corresponds to the position of the horizon and $q$ is related to the charge density carried by the horizon. The temperature of the dual plasma is given by

$$
T=\frac{d}{4 \pi z_{\mathrm{h}}}\left(1-\frac{d-2}{d} z_{\mathrm{h}}^{2} q^{2}\right)
$$

\footnotetext{
${ }^{7}$ The normalization of the gauge field term is typically determined by the microscopic details of the holographic construction - see discussion in [40]. Here, we simply chose the factor of $L^{2}$ for convenience. Further we note that in the case $d=2$, the following solution is modified by logarithmic terms.
} 
and the chemical potential is given by the asymptotic value of the gauge potential, i.e.,

$$
\mu=\lim _{z \rightarrow 0} A_{0}=\sqrt{\frac{2(d-1)}{d-2}} q .
$$

Since the CFT plasma is at rest, eq. (2.5) reduces to $\left\langle T_{\mu \nu}\right\rangle=\varepsilon \operatorname{diag}(1,1 /(d-$ $1), 1 /(d-1), \cdots)$ and the usual holographic prescription yields $[35,36]$

$$
\varepsilon=\frac{d-1}{2} \frac{L^{d-1}}{\ell_{\mathrm{P}}^{d-1}} \frac{1}{z_{\mathrm{h}}^{d}}\left(1+z_{\mathrm{h}}^{2} q^{2}\right)
$$

Now we wish to evaluate the change in the expectation value of the modular Hamiltonian produced by going to this new state. Since the energy density is again uniform, evaluating $\langle H\rangle_{1}$ is precisely the same calculation as in eq. (2.10), up to the additional overall factor appearing in eq. (2.28). Hence, we arrive at

$$
\Delta\left\langle H^{\prime \prime}\right\rangle=\Delta\langle H\rangle\left(1+z_{\mathrm{h}}^{2} q^{2}\right)
$$

where $\Delta\langle H\rangle$ is the result given in eq. (2.11).

Further since the black brane is static, the extremal surface appearing in the holographic entanglement entropy (1.1) again has a spherically symmetric profile $z=z(r)$ for a spherical entangling surface. Hence with the metric (2.23), the entropy functional becomes

$$
S_{1}^{\prime \prime}=2 \pi \frac{L^{d-1}}{\ell_{\mathrm{P}}^{d-1}} \Omega_{d-2} \int_{0}^{R} d r \frac{r^{d-2}}{z^{d-1}} \sqrt{1+\frac{\left(\partial_{r} z\right)^{2}}{h(z)}},
$$

where $h(z)$ is given in eq. (2.24). In proceeding, we again limit our analysis to a perturbative calculation with $R / z_{\mathrm{h}} \ll 1$ but we treat $z_{\mathrm{h}} q=O(1)$. Further, as before, the leading contribution to the change in the entropy comes from simply evaluating eq. (2.30) with the vacuum profile $z=z_{0}(r)$ and expanding in $R / z_{\mathrm{h}}$. However, we would like to refine our previous arguments. Here as in the previous examples, the leading changes to the asymptotic metric are $O\left(z^{d} / z_{\mathrm{h}}^{d}\right)$ and so we will find the leading change in the entropy is $\Delta S^{\prime \prime}=O\left(R^{d} / z_{\mathrm{h}}^{d}\right)$. The leading change of the profile of the extremal surface, $\delta z$, is also controlled by these leading changes in the metric. However, as we argued before, the entropy is only changed at quadratic order in $\delta z$ and hence we will find that this contribution produces a change in the entropy $\Delta S^{\prime \prime}\left(\delta z^{2}\right)=O\left(R^{2 d} / z_{\mathrm{h}}^{2 d}\right)-$ see section 3.2 for an explicit calculation. Hence at this point, we note that the next-toleading order changes in the above metric 2.23 are $O\left(z^{2 d-2} / z_{\mathrm{h}}^{2 d-2}\right)$ since we consider $z_{\mathrm{h}} q$ to be order 1 . If we calculate with these changes in the metric and the original profile, there will be an additional contribution to the change in the entropy at $O\left(R^{2 d-2} / z_{\mathrm{h}}^{2 d-2}\right)$ — this is verified by our calculation below. This contribution is still lower order in the 
$R / z_{\mathrm{h}}$ expansion compared to those arising from the change in the profile. Hence it is legitimate to consider this contribution without concerning ourselves with the change in the profile of the extremal surface. Therefore we expand eq. (2.30) as

$$
\begin{aligned}
\Delta S^{\prime \prime} & =\left.\pi \frac{L^{d-1}}{\ell_{\mathrm{P}}^{d-1}} \Omega_{d-2} \int_{0}^{R} d r \frac{r^{d-2} z\left(\partial_{r} z\right)^{2}}{z_{\mathrm{h}}^{d} \sqrt{1+\left(\partial_{r} z\right)^{2}}}\left[\left(1+z_{\mathrm{h}}^{2} q^{2}\right)-q^{2} \frac{z^{d-2}}{z_{\mathrm{h}}^{d-4}}\right]\right|_{z=z_{0}(r)} \\
& =\Delta S\left(1+z_{\mathrm{h}}^{2} q^{2}\right)-\pi \frac{L^{d-1}}{\ell_{\mathrm{P}}^{d-1}} \Omega_{d-2} \frac{q^{2}}{z_{\mathrm{h}}^{2 d-4} R} \int_{0}^{R} d r r^{d}\left(R^{2}-r^{2}\right)^{\frac{d-2}{2}} \\
& =\Delta S\left(1+z_{\mathrm{h}}^{2} q^{2}\right)-\frac{d-1}{2} \pi^{\frac{d+1}{2}} \frac{\Gamma(d / 2)}{\Gamma\left(d+\frac{1}{2}\right)} \frac{L^{d-1}}{\ell_{\mathrm{P}}^{d-1}}\left(z_{\mathrm{h}} q\right)^{2} \frac{R^{2 d-2}}{z_{\mathrm{h}}^{2 d-2}}
\end{aligned}
$$

where $\Delta S$ corresponds to the variation given in eq. (2.13).

Recall that we found $\Delta\langle H\rangle=\Delta S$ in eq. (2.14). Hence in comparing to eqs. (2.29) and (2.31), we find that the leading order terms are again equal, however, including the contribution at $O\left(R^{2 d-2} / z_{0}^{2 d-2}\right)$ yields

$$
\Delta\left\langle H^{\prime \prime}\right\rangle>\Delta S^{\prime \prime} .
$$

Hence we find that adding the chemical potential introduces a next-to-leading contribution which ensures that the relative entropy is positive. Using the above expressions, we have

$$
S\left(\rho_{1} \mid \rho_{0}\right) \simeq \frac{\pi}{2} \frac{L^{d-1}}{\ell_{\mathrm{P}}^{d-1}} \Omega_{d-2} \frac{\Gamma(d / 2) \Gamma((d+1) / 2)}{\Gamma\left(d+\frac{1}{2}\right)}\left(z_{\mathrm{h}} q\right)^{2} \frac{R^{2 d-2}}{z_{\mathrm{h}}^{2 d-2}}
$$

Since $S\left(\rho_{1} \mid \rho_{0}\right) \propto R^{2 d-2}$, we can trivially verify that the relative entropy also satisfies the monotonicity property (1.13), i.e., $\partial_{R} S\left(\rho_{1} \mid \rho_{0}\right)>0$. Using eqs. (2.26) and (2.27), it is straightforward to re-express the right-hand side as a function of the temperature and chemical potential. While the full expression is not particularly insightful, note that in the regime $1 \gg \mu / T \gg R T$, we have $S\left(\rho_{1} \mid \rho_{0}\right) \sim(R T)^{2 d-2}(\mu / T)^{2}$ and so, in particular, we observe that this nonvanishing contribution begins at quadratic order in the chemical potential.

In closing, we note that the result in eq. (2.31) was generated by a first-order deformation in the asymptotic metric, however, the latter is produced by the backreaction of the gauge field on the geometry and so the leading change in the relative entropy is quadratic in the corresponding coefficient $q$.

\section{General analysis}

In this section, we would like to generalize the previous analysis to examine the inequality (1.8) for more general holographic states. As long as we focus our attention on 
a spherical entangling surface, it is straightforward to evaluate $\Delta\langle H\rangle$ using eq. (1.10) since a standard holographic prescription allows us to determine $\left\langle T_{\mu \nu}\right\rangle[35,36]$. In principle, the calculation of the entanglement entropy using eq. (1.1) is more challenging because we must determine the extremal surface in the bulk geometry describing the second state $\rho_{1}$. However, as we saw above, if this state describes a 'small' perturbation of the initial vacuum state $\rho_{0}$, our calculations are restricted to considering asymptotic perturbations of the AdS geometry. Hence, our analysis of the holographic entanglement entropy was greatly simplified in this perturbative context. It also suggests that it is natural to formulate these calculations in the framework of the asymptotic Fefferman-Graham (FG) expansion [41] — see also [35]. In particular, such an approach will allow us to consider a much broader class of perturbed states without concerning ourselves with the details of the bulk geometry in the far infrared.

Using the FG expansion, we consider three distinct calculations in the following: We begin by considering states described by purely gravitational excitations in the AdS bulk. That is, the stress tensor is the only operator that has a nonvanishing expectation value in these states. Now let us introduce a small perturbative parameter $\alpha$ which controls the magnitude of $\left\langle T_{\mu \nu}\right\rangle$. Our first result is to demonstrate that we always saturate the inequality (1.8), i.e., $\Delta\langle H\rangle=\Delta S$, when working to linear order in $\alpha$. We emphasize that this equality holds even when $\left\langle T_{\mu \nu}\right\rangle$ varies on scales comparable to $R$, the size of the spherical entangling surface. Secondly, we extend these calculations to second order in $\alpha$ in section 3.2. There while $\Delta\langle H\rangle$ is unchanged, we show that the additional contributions to the entanglement entropy have a definite sign ensuring that $\Delta\langle H\rangle>\Delta S$. The third case, which we consider in section 3.3, involves states in which additional matter fields are excited in the dual AdS spacetime and hence additional operators acquire expectation values. As we saw in section 2.2 , it is relatively easy to determine quadratic corrections to the entanglement entropy coming from such perturbations. Below, we extend this analysis to a much broader class of states and verify that the quadratic contributions again ensure that $\Delta\langle H\rangle>\Delta S$.

As commented above, our general analysis will be formulated in the context of the Fefferman-Graham expansion of the asymptotic bulk solutions [35, 41]. Hence we begin by considering a general bulk metric written in FG coordinates

$$
d s^{2}=\frac{L^{2}}{z^{2}}\left(d z^{2}+g_{\mu \nu}\left(z, x^{\mu}\right) d x^{\mu} d x^{\nu}\right)
$$

We are considering the asymptotic geometry where $z \simeq 0$. We will always choose the asymptotic metric (on which the boundary CFT is defined) to be flat and so we may write

$$
g_{\mu \nu}\left(z, x^{\mu}\right)=\eta_{\mu \nu}+\delta g_{\mu \nu}\left(z, x^{\mu}\right)
$$


where $\delta g_{\mu \nu}$ begins with terms of order $z^{d}$. We are interested in calculations of holographic entanglement entropy (1.1) and so we will want to evaluate the area of various extremal surfaces in the bulk. In principle, for situations where the background geometry is not static, the profile of these $(d-1)$-dimensional surfaces would be specified by giving both the radial position and time in the bulk as functions of the remaining spatial coordinates, i.e., $z=z\left(x^{i}\right)$ and $t=t\left(x^{i}\right)$. However, our goal is to evaluate the change in the entanglement entropy $\Delta S$ and discussed in section 2.1, it will suffice to consider bulk surfaces that live in a constant time slice. Hence with a radial profile $z=z\left(x^{i}\right)$ alone, the induced metric $h_{i j}$ on this surface is given by

$$
h_{i j} d x^{i} d x^{j}=\frac{L^{2}}{z^{2}}\left(g_{i j}+\partial_{i} z \partial_{j} z\right) d x^{i} d x^{j}
$$

and the corresponding area is then

$$
A=L^{d-1} \int d^{d-1} x \sqrt{h}=L^{d-1} \int d^{d-1} x \sqrt{\operatorname{det} g_{i j}} \sqrt{1+g^{i j} \partial_{i} z \partial_{j} z} .
$$

In principle, eq. (3.4) can now be used as an effective action to determine the extremal profile $z=z\left(x^{i}\right)$. However, as before, to determine the leading change $\Delta S$, we will be evaluating the area in the new background geometry with the original profile (2.2).

\subsection{Linear corrections to relative entropy}

We begin by considering states $\rho_{1}$ whose small deviation of the vacuum state $\rho_{0}$ is characterized by an expectation value of stress tensor $T_{\mu \nu}^{0}$ in the boundary CFT. ${ }^{8}$ We suppose the latter is 'very small' and that the smallness is characterized by a (dimensionless) parameter $\alpha \ll 1$. As before, we will limit our attention to a spherical entangling surface for which the (vacuum) modular Hamiltonian (1.10) is linear in the stress tensor and so $\Delta\langle H\rangle$ is linear in $\alpha$. However, in eq. (1.8), the change in the entanglement entropy will receive contributions at all orders in $\alpha$. In the present section, we will only evaluate $\Delta S$ to linear order in $\alpha$.

In general, using the FG expansion, the deviation of the bulk metric from pure AdS in eq. (3.2) takes the form.

$$
\delta g_{\mu \nu}=\frac{2}{d} \frac{\ell_{\mathrm{P}}^{d-1}}{L^{d-1}} z^{d} \sum_{n=0} z^{2 n} T_{\mu \nu}^{(n)} .
$$

The bulk Einstein equations will determine $T_{\mu \nu}^{(n)}$ for $n>0$ in terms of expectation value $T_{\mu \nu}^{(0)}$. Following the above discussion, our strategy will be to only solve for $T_{\mu \nu}^{(n)}$ to leading order in $\alpha$ (or to linear order in $T_{\mu \nu}^{(0)}$ ).

\footnotetext{
${ }^{8}$ For simplicity, we drop the angle brackets in denoting this expectation value throughout our calculations here.
} 
Before we solve the Einstein equations, we let us recall that the goal is to evaluate the change in the holographic entanglement entropy in the perturbed metric. Here, we may apply the same reasoning as in section 2. In particular, in the vacuum AdS, there is an analytic solution (2.2) for the extremal bulk surface corresponding to a spherical entangling surface of radius $R$ in the boundary

$$
z_{0}^{2}+r^{2}=R^{2}, \quad \text { where } r^{2}=\sum_{i=1}^{d-1} x_{i}^{2} .
$$

Now in the perturbed background, the bulk entangling surface can also be given as an expansion in $\alpha$, i.e., $z\left(x^{i}\right)=z_{0}\left(x^{i}\right)+\alpha z_{1}\left(x^{i}\right)+\cdots$. However, as described in the previous section, since the profile $z_{0}$ is extremal to leading order, the perturbation $z_{1}$ only contributes at order $\alpha^{2}$. Hence we can evaluate the linear change in the area by simply evaluating the area (3.4) with the original profile $z_{0}$ in the perturbed background. Hence given (3.5), one finds to linear order in $\alpha$ that

$$
\Delta S=2 \pi \frac{\Delta A}{\ell_{\mathrm{P}}^{d-1}}=\frac{2 \pi R}{d} \int_{|x| \leq R} d^{d-1} x \sum_{n=0} z_{0}^{2 n}\left(T_{i}^{(n)}{ }_{i}^{i}-T^{(n)}{ }_{i j} \frac{x^{i} x^{j}}{R^{2}}\right) .
$$

Now we return to solving the Einstein equations, which can be written as

$$
\hat{R}_{A B}-\frac{1}{2} G_{A B}\left(\hat{R}+\frac{d(d-1)}{L^{2}}\right)=0
$$

where $\hat{R}_{A B}$ is the bulk Ricci tensor evaluated on the bulk metric $G_{A B}$ given as in eq. (3.1). Using the results from [42], we can write to linear order in $\alpha$,

$$
\begin{aligned}
\hat{R}_{\rho \rho} & =-\frac{d}{4 \rho^{2}}-\frac{1}{2} \partial_{\rho}^{2} \delta g_{\mu}^{\mu} \\
\hat{R}_{\mu \rho} & =\frac{1}{2}\left(\partial_{\rho} \partial_{\nu} \delta g_{\mu}^{\nu}-\partial_{\mu} \partial_{\rho} \delta g_{\nu}^{\nu}\right) \\
\hat{R}_{\mu \nu} & =R_{\mu \nu}-2 \rho \partial_{\rho}^{2} \delta g_{\mu \nu}+(d-2) \partial_{\rho} \delta g_{\mu \nu}+\eta_{\mu \nu} \partial_{\rho} \delta g_{\gamma}{ }^{\gamma}-\frac{d}{\rho}\left(\eta_{\mu \nu}+\delta g_{\mu \nu}\right) \\
\hat{R} & =-d(d+1)+\rho R+2(d-1) \rho \partial_{\rho} \delta g_{\mu}{ }^{\mu}-4 \rho^{2} \partial_{\rho}^{2} \delta g_{\mu}{ }^{\mu}
\end{aligned}
$$

where we have chosen a (dimensionless) radial coordinate $\rho=z^{2} / L^{2}$. Also, $R_{\mu \nu}$ and $R$ are curvature tensors evaluated on $g_{\mu \nu}$ treating $z$ (or $\rho$ ) as an external parameter. Explicitly, then the linear order in $\alpha$, we have

$$
R_{\mu \nu}=\frac{1}{2}\left(\partial_{\nu} \partial_{\gamma} \delta g_{\mu}^{\gamma}+\partial_{\mu} \partial_{\gamma} \delta g_{\nu}^{\gamma}-\square \delta g_{\mu \nu}-\partial_{\mu} \partial_{\nu} \delta g_{\gamma}^{\gamma}\right)
$$


Substituting eq. (3.5) and the above expression into the Einstein equations, we obtain the following equations for $T^{(n)}$ using the $\rho \rho$ and $\mu \rho$ components, respectively:

$$
\begin{aligned}
& \partial^{\mu} \partial^{\nu} T_{\mu \nu}^{(n)}-\square T_{\mu}^{(n) \mu}+(d-1)(d+2 n+2) T_{\mu}^{(n+1) \mu_{\mu}}=0, \quad T_{\mu}^{(0) \mu}=0, \\
& \partial_{\nu} T_{\mu}^{(n)}{ }_{\mu}^{\nu}-\partial_{\nu} T_{\mu}^{(n) \mu}=0 .
\end{aligned}
$$

Together, these two equations imply that

$$
T_{\mu}^{(n) \mu}=0, \quad \partial_{\nu} T_{\mu}^{(n)}{ }_{\mu}^{\nu}=0,
$$

for all $n$. Hence we note that Einstein equations automatically ensure that $T^{(n)}$ is traceless and conserved for all $n$. Finally, the $\mu \nu$ components of Einstein equations then reduce to

$$
T_{\mu \nu}^{(n)}=-\frac{\square T_{\mu \nu}^{(n-1)}}{2 n(d+2 n)}
$$

which implies

$$
T_{\mu \nu}^{(n)}=\frac{(-1)^{n} \Gamma[d / 2+1]}{2^{2 n} n ! \Gamma[d / 2+n+1]} \square^{n} T_{\mu \nu}^{(0)} .
$$

Of course, we can substitute these results back into eq. (3.7) to express $\Delta S$ entirely in terms of $T_{\mu \nu}^{(0)}$.

For the following, it will be more convenient to express the stress tensor in a Fourier expansion

$$
T_{\mu \nu}^{(0)}(x)=\int d^{d} p \exp (-i p \cdot x) \widehat{T}_{\mu \nu}(p) .
$$

Using the previous results, the change in the entanglement entropy (3.7) then becomes

$$
\begin{aligned}
& \Delta S=\frac{2 \pi R}{d} \int d^{d-1} x \int d^{d} p \exp (-i p \cdot x) \times \\
& \frac{\Gamma[d / 2+1]}{\left(z_{0}|p| / 2\right)^{d / 2}} \sum_{n=0}\left[\frac{1}{n ! \Gamma[d / 2+n+1]}\left(\frac{|p| z_{0}}{2}\right)^{2 n+d / 2}\right]\left(\widehat{T}_{i}^{i}(p)-\widehat{T}_{i j}(p) \frac{x^{i} x^{j}}{R^{2}}\right),
\end{aligned}
$$

where $|p|=\left|\sqrt{p_{\mu} p^{\mu}}\right|$. Now we may recognize that the sum in the square brackets yields precisely

$$
\sum_{n=0}\left[\frac{1}{n ! \Gamma[d / 2+n+1]}\left(\frac{|p| z_{0}}{2}\right)^{2 n+d / 2}\right]=I_{d / 2}\left(|p| z_{0}\right) .
$$

For time-like momenta $p$ in Lorentzian signature, it gives instead $J_{d / 2}\left(|p| z_{0}\right)$. That is, we recover an expression that is precisely proportional to the Green's function of the graviton in $\mathrm{AdS}_{d+1}$. However, note that the asymptotic boundary condition is taken to be one where the leading constant term is set to zero - for example, see [68]. The latter can be contrasted with the usual bulk-to-boundary Green's function which is proportional to $K_{d / 2}\left(|p| z_{0}\right)$, where the boundary condition is chosen such that the leading term near the AdS boundary is a constant. 
Saturating the inequality in eq. (1.8):

Turning to eq. (1.8), we would like to establish that this inequality is in fact saturated at linear order in $\alpha$ for the general class of states considered here. Given the modular Hamiltonian (1.10) (for a spherical entangling surface), we may write

$$
\Delta\langle H\rangle=\frac{\pi}{R} \int_{|x| \leq R} d^{d-1} x z_{0}^{2} T_{00}^{(0)} .
$$

where $z_{0}$ is the extremal profile in eq. (3.6). A priori, this expression bares no resemblance to the expression for $\Delta S$ in eq. (3.7), even after we substitute in the results in eq. (3.15).

To prove the inequality (1.8) is saturated, we begin by examining eq. (3.17) for a single momentum component with the corresponding quantity in $\delta H$ above. We can set the spatial direction of momentum in direction $x^{1}$, i.e.,

$$
T_{\mu \nu}^{(0)}(x)=\widehat{T}_{\mu \nu} e^{-i p \cdot x}
$$

We take the momentum to be time-like for definiteness. An analogous calculation holds for space-like momentum.

Conservation and tracelessness of $T_{\mu \nu}^{(0)}$ imply

$$
\widehat{T}_{i}^{i}=\widehat{T}_{00}, \quad \widehat{T}_{10}=-\frac{p^{0}}{p^{1}} \widehat{T}_{00} \quad \text { and } \quad \widehat{T}_{11}=\frac{\left(p^{0}\right)^{2}}{\left(p^{1}\right)^{2}} \widehat{T}_{00} .
$$

Then we note that given the stress tensor chosen in eq. (3.20), the integral of eq. (3.17) is symmetric under rotations leaving $x^{1}$ fixed. This implies the integral containing the term $\widehat{T}_{i j} x^{i} x^{j}$ will vanish for $i \neq j$. Also for $i=j=2, \cdots,(d-2)$, all the integrals are equal. Then inside the integral, we can replace

$$
\begin{gathered}
\widehat{T}_{i}^{i}-\widehat{T}_{i j} \frac{x^{i} x^{j}}{R^{2}} \rightarrow \widehat{T}_{00}-\widehat{T}_{11} \frac{\left(x^{1}\right)^{2}}{R^{2}}-\sum_{i=2}^{d-2} \widehat{T}_{i i} \frac{\left(x^{i}\right)^{2}}{R^{2}} \\
\rightarrow \widehat{T}_{00}-\widehat{T}_{11} \frac{\left(x^{1}\right)^{2}}{R^{2}}-\sum_{i=2}^{d-2} \widehat{T}_{i i} \frac{\sum_{j=2}^{d-2}\left(x^{j}\right)^{2}}{(d-2) R^{2}} \rightarrow \widehat{T}_{00}-\widehat{T}_{11} \frac{\left(x^{1}\right)^{2}}{R^{2}}-\sum_{i=2}^{d-2} \widehat{T}_{i i} \frac{r^{2}-\left(x^{1}\right)^{2}}{(d-2) R^{2}} \\
\left.\rightarrow \widehat{T}_{00}-\widehat{T}_{11} \frac{\left(x^{1}\right)^{2}}{R^{2}}-\left(\widehat{T}_{i}^{i}-\widehat{T}_{11}\right) \frac{r^{2}-\left(x^{1}\right)^{2}}{(d-2) R^{2}}\right) \\
\rightarrow \widehat{T}_{00}\left(1-\frac{\left(p^{0}\right)^{2}}{\left(p^{1}\right)^{2}} \frac{\left(x^{1}\right)^{2}}{R^{2}}-\frac{\left(1-\frac{\left(p^{0}\right)^{2}}{\left(p^{1}\right)^{2}}\right)\left(r^{2}-\left(x^{1}\right)^{2}\right)}{(d-2) R^{2}}\right)
\end{gathered}
$$

In the last transformation we have used (3.21). This final expression depends only on $\widehat{T}_{00}$, which is necessary for the equality with $\Delta\langle H\rangle$. 
Then $\Delta S$ reads in polar coordinates

$$
\begin{aligned}
\Delta S= & \frac{2^{(d+2) / 2} \pi R}{d|p|^{d / 2}} \Gamma[d / 2+1] \Omega_{d-3} \widehat{T}_{00} e^{i p^{0} t} \int_{0}^{R} d r r^{d-2} \int_{0}^{\pi} d \theta \sin ^{d-3} \theta e^{-i p^{1} r \cos (\theta)} \\
& \times \frac{J_{d / 2}\left(|p| \sqrt{R^{2}-r^{2}}\right)}{\left(R^{2}-r^{2}\right)^{d / 4}}\left(1-\frac{\left(p^{0}\right)^{2}}{\left(p^{1}\right)^{2}} \frac{r^{2} \cos ^{2} \theta}{R^{2}}-\frac{\left(1-\frac{\left(p^{0}\right)^{2}}{\left(p^{1}\right)^{2}}\right) r^{2} \sin ^{2} \theta}{(d-2) R^{2}}\right)
\end{aligned}
$$

The integrals over $\theta$ can then be done explicitly using

$$
\int_{0}^{\pi} d \theta \sin ^{q}(\theta) e^{-i x \cos (\theta)}=2^{q / 2} \sqrt{\pi} \Gamma[(q+1) / 2] \frac{J_{q / 2}(|x|)}{|x|^{q / 2}} .
$$

Now for the variation of the modular Hamiltonian, we substitute eq. (3.20) into the eq. (3.19) which yields

$$
\begin{aligned}
\Delta\langle H\rangle & =2 \pi \Omega_{d-3} \widehat{T}_{00} e^{i p^{0} t} \int_{0}^{R} d r r^{d-2} \int_{0}^{\pi} d \theta \sin ^{d-3} \theta \frac{R^{2}-r^{2}}{2 R} e^{-i p_{1} r \cos (\theta)} \\
& =2^{(d-1) / 2} \pi^{3 / 2} \Omega_{d-3} \Gamma[(d-2) / 2] \widehat{T}_{00} e^{i p^{0} t} \frac{R^{(d-1) / 2}}{\left|p^{1}\right|^{(d+1) / 2}} J_{(d+1) / 2}\left(\left|p^{1}\right| R\right) .
\end{aligned}
$$

Note the integral for $\Delta S$ in eq. (3.24) depends on an additional parameter $|p|$ which is not present in the integral in eq. (3.26). Then the equality between $\Delta S$ and $\Delta\langle H\rangle$ requires that the expression in eq. (3.24) is miraculously independent of $p$ for a given fixed value of $p^{1}$. One can check this actually happens by making an expansion in powers of $p$ and $p^{1}$ and also replacing $\left(p^{0}\right)^{2}=p^{2}+\left(p^{1}\right)^{2}$ in the integral in eq. (3.24). Collecting the terms with the same powers of $p$ and $p^{1}$, one arrives at expressions which are possible to integrate in $\theta$ and $r$ analytically. The result is that the coefficient of $\left(p^{1}\right)^{m} p^{n}$ in the expansion of $\Delta S$ is zero for any $n>0$. Hence, we may take the limit of $p \rightarrow 0$ in the integrand to simplify the calculation and eq. (3.24) becomes

$$
\begin{aligned}
\delta S & =\frac{4 \pi}{d} \Omega_{d-3} \widehat{T}_{00} e^{i p^{0} t} \int_{0}^{R} d r r^{d-2} \int_{0}^{\pi} d \theta \sin ^{d-3} \theta \frac{R^{2}-r^{2} \cos (\theta)^{2}}{2 R} e^{-i p_{1} r \cos (\theta)} \\
& =2^{(d-1) / 2} \pi^{3 / 2} \Omega_{d-3} \Gamma[(d-2) / 2] \widehat{T}_{00} e^{i p^{0} t} \frac{R^{(d-1) / 2}}{\left|p^{1}\right|^{(d+1) / 2}} J_{(d+1) / 2}\left(\left|p^{1}\right| R\right) .
\end{aligned}
$$

Now comparing eqs. (3.26) and (3.27), we see

$$
\Delta\langle H\rangle=\Delta S
$$

While this analysis was done for a single plane wave (3.20), since we are considering linear perturbations, the same equality must hold for a general Fourier expansion (3.16). Therefore, we conclude that eq. (3.28) holds for any first order perturbation of the stress tensor. In particular, this equality still applies even when $T_{\mu \nu}^{(0)}$ varies on scales comparable to $R$, the size of the spherical entangling surface. 


\subsection{Quadratic corrections to relative entropy}

While it was technically difficult to establish, the equality in eq. (3.28) should have been expected given the discussion preceding eq. (1.12). Similarly, if we extend the previous calculation of $\Delta S$ to second order in $\alpha$, we should expect that the new contributions at this order result in the required inequality (1.8). In this section, we verify that this expectation is indeed correct. For simplicity, we will restrict our attention to constant stress tensors.

To obtain the quadratic correction to the relative entropy, we proceed in three steps: First, we expand the bulk metric to quadratic order in the stress tensor. Then we expand the area functional (3.4) to quadratic order in the perturbation parameter $\alpha$. In particular, we obtain the equations of motion governing the deformation of the minimal surface to linear order in the stress tensor. Then solving the equations of motion, we substitute the results back into the area functional and collect the aggregate quadratic correction in the relative entropy.

\section{Step 1: Bulk metric}

In eq. (3.5) the bulk metric is expanded to linear order. To quadratic order, the expansion will take the from

$$
\delta g_{\mu \nu}=\eta_{\mu \nu}+a z^{d} T_{\mu \nu}+a^{2} z^{2 d}\left(n_{1} T_{\mu \alpha} T_{\nu}^{\alpha}+n_{2} \eta_{\mu \nu} T_{\alpha \beta} T^{\alpha \beta}\right)+\cdots
$$

where

$$
a=\frac{2}{d} \frac{\ell_{\mathrm{P}}^{d-1}}{L^{d-1}}
$$

The term, which is quadratic in the stress tensor, has the most general form allowed by Lorentz invariance, symmetry between $\mu$ and $\nu$, and that the trace of $T_{\mu \nu}$ vanishes. ${ }^{9}$ Further the power of $z^{2 d}$ in this term is simply determined by dimensional grounds. It remains to fix the coefficients $n_{1,2}$, which can be done by comparing this expression to the black brane metric (2.6) when the latter is re-expressed in FG coordinates (3.1). The latter requires transforming to a new radial coordinate in the asymptotic AdS geometry

$$
\tilde{z}=z\left(1+\frac{1}{2 d} \frac{z^{d}}{z_{\mathrm{h}}^{d}}+\frac{2+3 d}{16 d^{2}} \frac{z^{2 d}}{z_{\mathrm{h}}^{2 d}}+\cdots\right) .
$$

This new coordinate is chosen to produce $G_{z z}=L^{2} / \tilde{z}^{2}$, as required in eq. (3.1). With this radial coordinate, the remaining metric components in the asymptotic expansion

\footnotetext{
${ }^{9}$ Recall that we are limiting our attention to $T_{\mu \nu}$ being a constant and hence the derivative terms (3.15), which appeared at linear order in $\alpha$ above, vanish here.
} 
take the form:

$$
\begin{aligned}
& g_{00}=-1+\frac{d-1}{d} \frac{\tilde{z}^{d}}{z_{\mathrm{h}}^{d}}-\frac{4 d^{2}-9 d+4}{8 d^{2}} \frac{\tilde{z}^{2 d}}{z_{\mathrm{h}}^{2 d}}+\cdots \\
& g_{i j}=\delta_{i j}\left(1+\frac{1}{d} \frac{\tilde{z}^{d}}{z_{\mathrm{h}}^{d}}-\frac{d-4}{8 d^{2}} \frac{\tilde{z}^{2 d}}{z_{\mathrm{h}}^{2 d}}+\cdots\right) .
\end{aligned}
$$

Recall that the stress tensor takes the form given in eqs. (2.7) and (2.8), as can be read off from the leading terms above. Then comparing eqs. (3.29) and (3.32), we can read off $n_{1}$ and $n_{2}$ as

$$
n_{1}=\frac{1}{2} \quad \text { and } \quad n_{2}=-\frac{1}{8(d-1)} .
$$

\section{Step 2: Expansion of area functional and equations of motion}

The profile of the extremal surface receives corrections since the bulk is altered. Recall from the previous section that the minimal surface in static gauge can be described by $z\left(x^{i}\right)$, i.e., the bulk radial coordinate is specified as a function of the spatial coordinates $x^{i}$. In the present perturbative construction, we can expand

$$
z\left(x^{i}\right)=z_{0}\left(x_{i}\right)+\alpha z_{1}\left(x_{i}\right)+\alpha^{2} z_{2}\left(x_{i}\right)+\cdots,
$$

where $z_{0}$ is given in eq. (3.6). Note that since we are only interested in quadratic corrections to the entanglement entropy, $z_{2}$ is not needed since it would appear linearly in the area functional and hence would vanish by virtue of equations of motion.

The order $\alpha^{2}$ correction to the area functional (3.4) can be written as

$$
A_{(2)}=A_{2,0}+A_{2,1}+A_{2,2}
$$

where we are separating the contributions into three terms, according to the power of $z_{1}$ appearing in the expressions, which is denoted by the second index. Only $A_{2,1}$ and $A_{2,2}$ contribute to the linearized equations of motion for $z_{1}$.

Carefully expanding, we find

$$
\begin{aligned}
A_{2,0} & =L^{d-1} a^{2} \int d^{d-1} x R z_{0}^{d}\left(-\frac{1}{16}\left(1-\frac{r^{2}}{(d-1) R^{2}}\right)\left(T_{00}^{2}+T_{i j} T^{i j}\right)\right. \\
& \left.+\frac{T_{i 0} T^{i 0}}{8}\left(1+\frac{r^{2}}{(d-1) R^{2}}\right)+\frac{x^{i} x^{k}}{4 R^{2}} T_{i \alpha} T_{k}^{\alpha}+\frac{1}{8}\left(T^{2}-T_{x}^{2}-2 T T_{x}\right)\right),
\end{aligned}
$$

where

$$
T \equiv T_{i}^{i} \quad \text { and } \quad T_{x} \equiv T_{i j} \frac{x^{i} x^{j}}{R^{2}}
$$


Note that we have made use of $z_{0}^{2}=R^{2}-r^{2}$ to simplify the above expression. ${ }^{10}$ Further, we find:

$$
\begin{array}{rl}
A_{2,1}=L^{d-1} & a \int d^{d-1} x \frac{R}{2 z_{0}}\left(T\left(z_{1}-\frac{z_{0}^{2}}{R^{2}} x^{i} \partial_{i} z_{1}\right)\right. \\
+ & \left.T_{i j}\left(\frac{2 z_{0}^{2} x^{i} \partial^{j} z_{1}}{R^{2}}-\frac{z_{1} x^{i} x^{j}}{R^{2}}-\frac{z_{0}^{2} x^{i} x^{j} x^{k} \partial_{k} z_{1}}{R^{4}}\right)\right),
\end{array}
$$

and

$$
\begin{aligned}
A_{2,2}=L^{d-1} & \int d^{d-1} x \frac{R}{z_{0}^{d}}\left(\frac{d(d-1) z_{1}^{2}}{2 z_{0}^{2}}+\frac{z_{0}^{2}\left(\partial z_{1}\right)^{2}}{2 R^{2}}\right. \\
& \left.-\frac{z_{0}^{2}\left(x^{i} \partial_{i} z_{1}\right)^{2}}{2 R^{4}}+\frac{(d-1)}{2} \frac{x^{i} \partial_{i} z_{1}^{2}}{R^{2}}\right)
\end{aligned}
$$

Note that in $A_{2,1}$, we have already dropped terms that vanish upon evaluating them on the minimal surface $z_{0}$. We also remind the reader that the boundary terms do not contribute. Now the equations of motion for $z_{1}$ are derived by varying $A_{2,1}+A_{2,2}$ and can be written as

$$
\frac{1}{z_{0}^{d-1} R}\left(\partial^{2}\left(z_{0} z_{1}\right)-\frac{x^{i} x^{j}}{R^{2}} \partial_{i} \partial_{j}\left(z_{0} z_{1}\right)\right)=\frac{z_{0}}{2 R}\left((d-2) T+(d+2) T_{x}\right) .
$$

The perturbation $z_{1}$ is expected to take the form $T f_{1}(r)+T_{i j} x^{i} x^{j} f_{2}(r)$. After some trial an error to solve for $f_{2}$, and setting the appropriate boundary conditions by adding suitable choice of solutions to the homogeneous equation, we arrive at the following very simple solution in general $d$ :

$$
z_{1}=-\frac{a R^{2} z_{0}^{d-1}}{2(d+1)}\left(T+T_{x}\right)
$$

\section{Step 3: Substitution into the area functional}

With all the ingredients in place, we are ready to substitute everything back into the area functional. This amounts to some more tedious algebra resulting in seven tensor structures:

$$
\begin{aligned}
A_{(2)}=L^{d-1} a^{2} \int d^{d-1} x & \left(c_{1} T^{2}+c_{2} T_{x}^{2}+c_{3} T_{i j}^{2}+\right. \\
& \left.c_{4} T_{i 0} T^{i 0}+c_{5} \frac{x^{i} T_{i j} T^{j}{ }_{k} x^{k}}{R^{2}}+c_{6} \frac{x^{i} T_{i 0} T^{0}{ }_{j} x^{j}}{R^{2}}+c_{7} T T_{x}\right) .
\end{aligned}
$$

\footnotetext{
${ }^{10}$ We emphasize that the Greek indices $\mu, \nu, \cdots$ run through all the indices corresponding to the flat boundary directions, whereas Latin indices $i, j, \cdots$ are restricted to the spatial directions.
} 
The coefficients are given by

$$
\begin{aligned}
c_{1}= & \frac{z_{0}^{d-4}}{16(d+1)^{2}(d-1) R}\left((d+1)^{2} r^{6}+\left(3+d\left(3 d^{2}+d-15\right)\right) r^{4} R^{2}\right. \\
& \left.\left.\quad+\left(d^{2}(13-8 d)+2 d\right)-3\right) r^{2} R^{4}+\left(3 d^{3}-7 d^{2}+d+3\right) R^{6}\right), \\
c_{2}= & \frac{z_{0}^{d-4}}{8(d+1)^{2}}\left(\left(1-5 d^{2}\right) r^{2} R^{3}+(d(4 d+3)-3) R^{5}\right), \\
c_{3}= & \frac{\left(\frac{r^{2}}{d-1}-R^{2}\right) z_{0}^{d}}{16 R}, \\
c_{4}= & \frac{\left(\frac{r^{2}}{d-1}+R^{2}\right) z_{0}^{d}}{8 R}, \\
c_{5}= & R z_{0}^{d} \frac{d(d-2)-1}{4(d+1)^{2}} \\
c_{6}= & \frac{R}{4} z_{0}^{d}, \\
c_{7}= & z_{0}^{d-4} \frac{R^{3}(d-1)}{4(d+1)^{2}}\left((1-3 d) r^{2}+(2 d+1) R^{2}\right) .
\end{aligned}
$$

Proceeding with the remaining integrals, it is useful to note that by symmetry, whenever an integral has the form $\int d^{d-1} x\left(x^{i} x^{j} x^{k} x^{l} \cdots\right) f(r)$, i.e., there are $n$ pairs of $x^{i}$ 's in the integrand, we can simply replace them by

$$
N\left(\delta_{i j} \delta_{k l} \cdots+\text { permutations }\right) \int d^{d-1} x r^{2 n} f(r),
$$

with some appropriate normalization constant $N$. Using this fact, we are left with a final result of the form

$$
A_{(2)}=a^{2} L^{d-1} \Omega_{d-2}\left(C_{1} T^{2}+C_{2} T_{i j}^{2}+C_{3} T_{0 i}^{2}\right)
$$

where

$$
\begin{aligned}
& C_{1}=-\frac{d \sqrt{\pi} R^{2 d} \Gamma[d+1]}{2^{d+4}(d+1) \Gamma\left[d+\frac{3}{2}\right]}, \\
& C_{2}=C_{1}, \\
& C_{3}=-\frac{(d+2) \sqrt{\pi} R^{2 d} \Gamma[d+1]}{2^{d+3}(d-1) \Gamma\left[d+\frac{3}{2}\right]} .
\end{aligned}
$$

Note that in the above expression, $T_{0 i}^{2} \equiv T_{0 i} T_{0 j} \delta^{i j} \geq 0$. Therefore given that the three coefficients are negative, we are assured that the second order perturbation to the area 
is negative and hence the second order contribution to the holographic entanglement entropy ensures that the inequality (1.8) is satisfied. Since at second order, we have $\Delta\langle H\rangle \neq \Delta S$, it is nontrivial to check the monotonicity property in eq. (1.13). However, from the above result, we find that $S\left(\rho_{1} \mid \rho_{0}\right) \propto R^{2 d}$ and hence this inequality is simply satisfied, i.e., $\partial_{R} S\left(\rho_{1} \mid \rho_{0}\right)>0$.

As an example, we might apply these general results to the static thermal gas described by the planar AdS black hole. The corresponding stress tensor is given by eqs. (2.7) and (2.8), ${ }^{11}$ i.e., we have $T_{00}=\varepsilon$ and $T_{i j}=\delta_{i j} \varepsilon /(d-1)$. The solution of eq. (3.40) can be written as

$$
z_{1}(r)=\frac{k_{1}}{\sqrt{R^{2}-r^{2}}}+a \varepsilon\left(\frac{\left((d-1) R^{d+2}-\left(R^{2}-r^{2}\right)^{d / 2}\left(r^{2}+(d-1) R^{2}\right)\right)}{2\left(d^{2}-1\right) \sqrt{R^{2}-r^{2}}}\right),
$$

where $k_{1}$ is an undetermined integration constant. To ensure that $r \rightarrow R$ as $z \rightarrow 0$, which is already satisfied by $z_{0}$, we must choose

$$
k_{1}=-\frac{a \varepsilon R^{d+2}}{2(d+1)} .
$$

This choice yields precisely the solution for $z_{1}$ given in eq. (3.41). Substituting this solution into the area functional, we find

$$
\Delta S_{(2)}=-\frac{\pi^{3 / 2} d \Omega_{d-2} \Gamma[d-1]}{2^{d+1}(d+1) \Gamma\left[d+\frac{3}{2}\right]} \frac{L^{d-1}}{\ell_{\mathrm{P}}^{d-1}} R^{2 d} \varepsilon^{2} .
$$

which as required is a negative contribution. One should appreciate the fact that the integrand involves a complicated collection of polynomials in $d$. However, the final result reduces to the above simple form.

\subsection{Corrections from additional operators}

To this point, we have only considered a special class of states that give rise to a nontrivial expectation value for the stress tensor. For generic perturbations away from the vacuum, we would expect that other operators will acquire nontrivial expectation values. Hence in this section, we consider states in which certain operators beyond the stress tensor acquire an expectation value. The dual description will involve bulk gravity solutions in which additional matter fields are excited. As we saw with the

\footnotetext{
${ }^{11}$ In keeping with the above analysis, we might introduce an explicit expansion parameter $\alpha$ to these expressions. However, we adopt the simpler approach of formally setting $\alpha=1$ in the above expansion. From our previous examination of the thermal bath, as well as the results here, we can infer that $\Delta S$ appears as an expansion in the small parameter $a R^{d} \varepsilon$.
} 
charge black brane in section 2.2, it is relatively easy to determine quadratic corrections to the entanglement entropy coming from such matter field perturbations. Below, we evaluate the analogous contributions to $\Delta S$ for two types of states: The first will involve a scalar operator acquiring an expectation value. The dual description involves adding a massive scalar field to the gravitational theory. The second class will involve perturbations by a conserved current in the boundary theory or a gauge field in the bulk. Hence analyzing these latter configurations is a simple generalization of that for the charged black brane. For both families of states, we find that the quadratic contributions again ensure that $\Delta\langle H\rangle>\Delta S$.

\section{Perturbing with a scalar condensate}

In our first class of states, a scalar operator $\mathcal{O}$ of dimension $\Delta$ acquires a non-trivial expectation value (in the absence of any sources). The corresponding dual description is that a scalar field has been turned on and subsequently back reacts on the geometry to change the entanglement entropy. We will limit ourselves here to calculate only the leading contribution of this back reaction. The bulk action, which we are considering here, is given by

$$
I=\frac{1}{2 \ell_{\mathrm{P}}^{d-1}} \int d^{d+1} x \sqrt{G}\left[R-\frac{1}{2}(\partial \phi)^{2}-V(\phi)\right] .
$$

Since we are only solving perturbatively in $\phi$, we need only to keep up to quadratic terms in the scalar, and thus the potential can be taken simply as

$$
V(\phi)=-\frac{d(d-1)}{L^{2}}+\frac{1}{2} m^{2} \phi^{2}
$$

where the first term provides the negative cosmological constant.

A standard result [43] in the AdS/CFT correspondence is that to leading order in the condensate, the scalar field $\phi$ of mass $m=\Delta(d-\Delta)$ behaves asymptotically as

$$
\phi=\gamma \mathcal{O} z^{\Delta}+\cdots
$$

with some normalization constant $\gamma$. This can be substituted into the Einstein equation which, in the presence of the scalar, can be written as

$$
\hat{R}_{A B}=\frac{1}{2} \partial_{A} \phi \partial_{B} \phi+\frac{1}{d-1} G_{A B} V(\phi) .
$$

In the presence of the scalar field, we expect that the boundary expansion of the metric is altered [35]. However, since we are only interested in the leading contribution of the perturbation, cross terms between the boundary stress tensor and the scalar 
condensates need not be included here. To linear order in the boundary stress tensor and quadratic order in the operator, the expansion of the metric $\delta g_{\mu \nu}$ in eq. (3.2) takes the form

$$
\delta g_{\mu \nu}=a z^{d} \sum_{n=0} z^{2 n} T_{\mu \nu}^{(n)}+z^{2 \Delta} \sum_{n=0} z^{2 n} \sigma_{\mu \nu}^{(n)}+\cdots,
$$

where, of course, terms in the first sum were analyzed in section (3.1). In both sums, the superscript $(n)$ indicates that the corresponding operator contains a total of $2 n$ derivatives, e.g., see eq. (3.15). Hence, for $n=0$, the only possible contribution of the scalar is $\sigma_{\mu \nu}^{(0)}=\alpha_{0} \eta_{\mu \nu} \mathcal{O}^{2}$ where $\alpha_{0}$ is some constant. The latter is easily determined by substituting the expansion of the metric and also that of the scalar field into the Einstein equations (3.59), which yields

$$
\sigma_{\mu \nu}^{(0)}=-\frac{\gamma^{2}}{4(d-1)} \eta_{\mu \nu} \mathcal{O}^{2}
$$

Note that the coefficient here is negative definite, which will be crucial in evaluating the change in the entanglement entropy below.

For the interested reader, we also consider the next term $\sigma_{\mu \nu}^{(1)}$, which carries two derivatives acting on the condensate $\mathcal{O}$. Demanding Lorentz invariance and symmetry in $\mu, \nu$, lets one to write the general form

$$
\sigma_{\mu \nu}^{(1)}=\alpha_{1} \partial_{\mu} \mathcal{O} \partial_{\nu} \mathcal{O}+\alpha_{2} \mathcal{O} \partial_{\mu} \partial_{\nu} \mathcal{O}+\alpha_{3} \eta_{\mu \nu} \mathcal{O} \square \mathcal{O}+\alpha_{4} \eta_{\mu \nu}(\partial \mathcal{O})^{2},
$$

with some undetermined coefficients $\alpha_{i}$. Again using the equations of motion (3.59), we arrive at:

$$
\begin{aligned}
\sigma_{\mu \nu}^{(1)}= & \frac{\gamma^{2}}{4(d-1)(\Delta+1)(2 \Delta+2-d)}\left((d-2) \mathcal{O} \partial_{\mu} \partial_{\nu} \mathcal{O}+\Delta \eta_{\mu \nu} \mathcal{O} \square \mathcal{O}\right) \\
& \left.-\left(d \partial_{\mu} \mathcal{O} \partial_{\nu} \mathcal{O}-\eta_{\mu \nu}(\partial \mathcal{O})^{2}\right)\right)
\end{aligned}
$$

For general $\mathcal{O}(x)$, we would have to consider the sums in eq. (3.60) to all orders in derivatives. However, if $\mathcal{O}$ is slowly varying on the scale of $R, \sigma^{(0)}$ provides the leading contribution to the change in the entanglement entropy and we focus on this scenario here. As in our previous calculations, we determine this leading contribution by evaluating the area functional (3.4) with the perturbed metric but the leading order profile (3.6) for the extremal surface. The resulting change in the entanglement entropy 
is given simply by

$$
\begin{aligned}
\Delta S(\mathcal{O}) & =\frac{\pi L^{d-1} R}{\ell_{\mathrm{P}}^{d-1}} \int \frac{d^{d-1} x}{z_{0}^{d-2 \Delta}}\left(\sigma^{(0) i}{ }_{i}-\sigma_{i j}^{0} \frac{x^{i} x^{j}}{R^{2}}\right) \\
& =-\frac{\pi \gamma^{2} L^{d-1} R}{4 \ell_{\mathrm{P}}^{d-1}} \mathcal{O}^{2} \int \frac{d^{d-1} x}{z_{0}^{d-2 \Delta}}\left(1-\frac{r^{2}}{(d-1) R^{2}}\right) \\
& =-\frac{\gamma^{2} L^{d-1}}{\ell_{\mathrm{P}}^{d-1}} \frac{\pi^{3 / 2}\left(\Delta-\frac{(d-2)^{2}}{2(d-1)}\right) \Gamma\left[\Delta-\frac{d}{2}+1\right]}{8 \Gamma\left[\Delta-\frac{d}{2}+\frac{5}{2}\right]} \Omega_{d-2} R^{2 \Delta} \mathcal{O}^{2} .
\end{aligned}
$$

Note that the unitarity bound $\Delta>\frac{d}{2}-1$ ensures that the numerical prefactor in the last line is positive and hence the overall result for $\Delta S$ is negative. We note that this overall minus sign descends directly from eq. (3.61). Hence it is interesting that at the level of the FG expansion, the metric appears to know already about the positivity of the relative entropy!

It is interesting to compare the above contribution of the scalar condensate $\mathcal{O}$ with the leading order contribution coming from the stress tensor. In particular, one might consider a scenario where the expectation value of both operators is set by a single scale $\mu$ (e.g., the temperature), in which case, we would have $\mathcal{O} \sim \mu^{\Delta}$ and $T_{\mu \nu} \sim \mu^{d}$. Then the corresponding contributions to the entropy would scale like $\Delta S(\mathcal{O}) \sim(R \mu)^{2 \Delta}$ and $\Delta S\left(T_{\mu \nu}\right) \sim(R \mu)^{d}$ where our calculations would hold in a regime where $R \mu \ll 1$. Hence if $\mathcal{O}$ is sufficiently relevant, i.e., $\frac{d}{2}-1<\Delta<\frac{d}{2}$, then its contribution would be the dominant contribution. Of course, with $\frac{d}{2}<\Delta<d$, the stress energy would

produce the dominant contribution while for the special case $\Delta=\frac{d}{2}$, the scaling of both contributions would be the same. In a more general situation where there are several scales in the problem, the scale of $T_{\mu \nu}$ would necessarily be related to that of $\mathcal{O}$ and then there would be no obvious way to compare their respective contributions to $\Delta S$.

It follows from the above expression (3.64) that relative entropy is proportional to $R^{2 \Delta}$ and hence it also satisfies the monotonicity inequality (1.13).

\section{Perturbing with a current}

Here we provide a brief description of the extension of the analysis in section 2.2 to a state with a general boundary current $J_{\mu}$. Recall first we wish to construct a metric in the FG form, as given in eqs. (3.1) and (3.2). For simplicity, we will assume that the expectation value of the current is constant and then to leading order the metric perturbation takes the form

$$
\delta g_{\mu \nu}=a z^{d} T_{\mu \nu}^{(0)}+z^{2 d-2}\left(b J_{\mu} J_{\nu}+c \eta_{\mu \nu} J^{2}\right)
$$


where the constants, $a, b$ and $c$, are all dimensionless. Since we are working to linear order in the metric perturbation, we can consider the contribution of each of the two terms in eq. (3.65) independently, as above for the scalar operator. We know that the $T_{\mu \nu}^{(0)}$ contribution saturates the inequality (1.8) and hence the current perturbations must produce a negative contribution to the change in the entanglement entropy.

Recall that $a$ is given in eq. (3.30). To determine the remaining constants, we compare to the charged black brane metric (2.23). It is convenient to write the metric function in eq. (2.24) as simply

$$
h=1-\gamma \tilde{z}^{d}+\beta \tilde{z}^{2 d-2},
$$

with $\gamma$ and $\beta$ being positive constants. We have to change the radial coordinate $z$ in order to put the metric in the desired FG form (3.1). After this is done, we find to leading order that the remaining metric components take the form

$$
\begin{aligned}
g_{00} & =-\left(1-\gamma\left(1-\frac{1}{d}\right) z^{d}+\beta\left(1-\frac{1}{2 d-2}\right) z^{2 d-2}\right) \\
g_{i j} & =\delta_{i j}\left(1+\gamma \frac{z^{d}}{d}-\beta \frac{z^{2 d-2}}{2 d-2}\right) .
\end{aligned}
$$

Setting $J_{i}=0$ in eq. (3.65), we may compare the resulting expression with the above and find:

$$
b=-2(d-1) c, \quad c=\frac{\beta}{2(d-1) J_{0}^{2}} .
$$

Further identifying $J_{0} \equiv \lim _{z \rightarrow 0} z^{d-3} \partial_{z} A_{0}$ in the charged black brane solution we find that $c$ as a positive constant independent of the current, i.e.,

$$
c=\frac{1}{4(d-1)^{2}(d-2)} .
$$

Now the relevant part of the metric perturbation becomes

$$
\delta g_{\mu \nu}=c z^{2 d-2}\left(-2(d-1) J_{\mu} J_{\nu}+\eta_{\mu \nu} J^{2}\right) .
$$

Inserting this expression into the area functional (3.4) yields

$$
\Delta S=\frac{\pi R L^{d-1}}{\ell_{\mathrm{P}}^{d-1}} \int d^{d-1} x \frac{1}{z_{0}^{d}}\left(\delta g_{i}{ }^{i}-\delta g_{i j} \frac{x^{i} x^{j}}{R^{2}}\right) .
$$

For a constant current, we then find that the integral yields

$$
\Delta S=-\frac{\pi^{3 / 2}(d-3) ! \Omega_{d-2}}{2^{d+1} \Gamma\left[d+\frac{1}{2}\right]} \frac{L^{d-1} R^{2 d-2}}{\ell_{\mathrm{P}}^{d-1}}\left(\vec{J}^{2}+\left(J^{0}\right)^{2}\right) .
$$

Then from eq. (3.72), it follows that relative entropy $\Delta\langle H\rangle-\Delta S$ is positive, and it also increasing as $R^{2 d-2}$, satisfying the monotonicity inequality (1.13). 


\subsection{Corrections for general entangling surfaces}

In this section, we consider extending our analysis to entangling surfaces, which are not simply spheres. Let us begin by considering the area functional (3.4) with a generic entangling surface in the boundary and a perturbation of the vacuum state in which the stress tensor is excited. At linear order, the perturbation of the bulk geometry still takes the form presented in eq. (3.5) where the coefficients $T_{\mu \nu}^{(n)}$ are given by eq. (3.15). As in our previous examples, the holographic calculation of the entanglement entropy in the AdS vacuum will yield some extremal profile $z_{0}\left(x^{i}\right)$ depending on the geometry of the entangling surface. Now while this profile is perturbed in the excited state, the perturbation will only contributes to the change in the area at second order. Hence we can evaluate the linear change of the area by simply evaluating the area (3.4) with the original profile $z_{0}$ in the perturbed background. Therefore with a generic entangling surface, the linear perturbation of the entanglement entropy becomes

$$
\Delta S=2 \pi \frac{\Delta A}{\ell_{\mathrm{P}}^{d-1}}=\frac{2 \pi}{d} \int d^{d-1} x \sqrt{1+\left(\partial z_{0}\right)^{2}} \sum_{n=0} z_{0}^{2 n+1}\left(T_{i}^{(n)}{ }_{i}^{i}-T^{(n)}{ }_{i j} \frac{\partial^{i} z_{0} \partial^{j} z_{0}}{1+\left(\partial z_{0}\right)^{2}}\right)
$$

where $\left(\partial z_{0}\right)^{2}=\delta^{i j} \partial_{i} z_{0} \partial_{j} z_{0}$ and implicitly, the boundary geometry is simply flat space. Previously we concluded in eq. (3.13) that all of the tensors $T_{\mu \nu}^{(n)}$ are traceless and hence we can replace $T_{i}^{(n)}{ }_{i}^{i}=T_{00}^{(n)}$, which in turn are all related to the local energy density $T_{00}^{(0)}$ by eq. (3.15). Hence the first term above is controlled entirely by the energy density. However, there is no clear connection to the energy density in the second term. In section 3.1, the rotational symmetry of the spherical entangling surface and the corresponding bulk profile (3.6) was essential in reducing this expression to a contribution which again was controlled by $T_{00}^{(0)}$. Hence our observation here is simply that we should expect other components of the stress tensor to contribute to $\Delta S$, even at linear order, for entangling surfaces with a less symmetric geometry.

To explicitly illustrate this behavior, we consider the well-studied case of a 'slab' geometry where the entangling surface is comprised of two flat planes at $x= \pm \ell / 2[17]$. The extremal surface in the AdS vacuum has a profile $z(x)$ and the area becomes

$$
A=L^{d-1} B^{d-2} \int_{-\ell / 2}^{\ell / 2} \frac{d x}{z^{d-1}} \sqrt{1+z^{\prime 2}}
$$

where $B$ is an IR length scale that regulates the size of the two planes, i.e., $B^{d-2}$ is the area of one plane. Further regarding this area as an action for $z(x)$, the profile is constrained by a conserved quantity [17]

$$
z^{d-1} \sqrt{1+z^{\prime 2}}=z_{*}^{d-1}
$$


Here $z_{*}$ is the maximum value of $z$ which the extremal surface reaches in the bulk at $x=0$,

$$
z_{*}=\frac{\Gamma\left[\frac{1}{2(d-1)}\right]}{2 \sqrt{\pi} \Gamma\left[\frac{d}{2(d-1)}\right]} \ell .
$$

The change in the entropy (3.73) then becomes

$$
\Delta S=\frac{2 \pi}{d} B^{d-2} z_{*}^{d-1} \int_{-\ell / 2}^{\ell / 2} d x \sum_{n=0} z^{2 n+2-d}\left[T_{00}^{(n)}-T_{x x}^{(n)}\left(1-\frac{z^{2(d-1)}}{z_{*}^{2(d-1)}}\right)\right] .
$$

Hence we see that both the energy density and the pressure along the $x$-axis are contributing in this result. To produce a more explicit result, we can simplify the calculation by assuming that the expectation value of the stress tensor is uniform, i.e., $T^{(n)}{ }_{\mu \nu}=0$ for $n \geq 1$. Then eq. (3.77) becomes

$$
\begin{aligned}
\Delta S & =\frac{2 \pi}{d} B^{d-2} z_{*}^{d-1} \int_{-\ell / 2}^{\ell / 2} \frac{d x}{z^{d-2}}\left[T_{00}-T_{x x}\left(1-\frac{z^{2(d-1)}}{z_{*}^{2(d-1)}}\right)\right] \\
& =\frac{\pi^{1 / 2} \Gamma\left[\frac{d}{d-1}\right] \Gamma\left[\frac{1}{2(d-1)}\right]^{2}}{8 d \Gamma\left[\frac{3 d-1}{2(d-1)}\right] \Gamma\left[\frac{d}{2(d-1)}\right]^{2}} B^{d-2} \ell^{2}\left[\left(\frac{d+1}{d-1}\right) T_{00}-T_{x x}\right],
\end{aligned}
$$

where we have used eqs. (3.75) and (3.76) to evaluate the final expression above. Here again, we see that the result contains a term proportional to $T_{x x}$.

Then we observe that with the first order calculations described here, we expect that the inequality (1.8) must be saturated, i.e., $\Delta\langle H\rangle=\Delta S$. Therefore from this result, we can also infer that the modular Hamiltonian for the slab geometry also contains terms which are linear in the operator $T_{x x}$. Hence from these calculations, we can begin to see the appearance of new operators, i.e., other components of the stress tensor beyond $T_{00}$, appearing in the modular Hamiltonian for regions with general entangling surfaces.

Let us add a few more observations about $\Delta S$ for general entangling surfaces. First, we note that if we make a Fourier transform of the stress tensor, as in eq. (3.16), then eq. (3.73) can be rewritten using eq. (3.18) as

$$
\begin{aligned}
\Delta S=\pi \Gamma[d / 2] \int d^{d-1} x & \int d^{d} p \exp (-i p \cdot x) \sqrt{1+\left(\partial z_{0}\right)^{2}} \\
& \frac{I_{d / 2}\left(|p| z_{0}\right)}{\left(z_{0}|p| / 2\right)^{d / 2}}\left(\widehat{T}_{00}(p)-\widehat{T}_{i j}(p) \frac{\partial^{i} z_{0} \partial^{j} z_{0}}{1+\left(\partial z_{0}\right)^{2}}\right),
\end{aligned}
$$

where $|p|=\left|\sqrt{p_{\mu} p^{\mu}}\right|$. Hence the same Green's function $I_{d / 2}\left(|p| z_{0}\right)$ appears in evaluating this leading contribution to $\Delta S$ for general entangling surfaces. Unfortunately, without 
the symmetry of a spherical entangling surface, this expression does not simplify in any obvious way.

In fact, eq. (3.73) makes an important assumption about the extremal surface in the bulk. Namely, that it is single-valued as a function of the boundary coordinates $x^{i}$ or alternatively, that the extremal surface does not extend to values of $x^{i}$ beyond the region $V$. Unfortunately, this assumption can be shown not to apply in many cases. For example, a standard FG-like expansion of the extremal surface describes the bulk surface as $X^{\mu}\left(y^{a}, z\right)$ where $y^{a}$ are coordinates along the entangling surface and $z$ is the usual radial coordinate in the bulk $[44,45]$. Then near the AdS boundary, one finds

$$
X^{i}=X_{0}^{i}\left(y^{a}\right)-\frac{1}{2(d-2)} K^{i}\left(y^{a}\right) z^{2}+\cdots
$$

where $X_{0}^{i}\left(y^{a}\right)$ describes the position of the entangling surface in the boundary and $K^{i}$ is the trace of the extrinsic curvature for the spatial normal to the entangling surface. Our conventions are such that $X^{i}<X_{0}^{i}\left(y^{a}\right)$ corresponds to the region inside the entangling surface and $K^{i}=+(d-2) X^{i} / R^{2}$ for a sphere of radius $R$, centered at $X^{i}=0$. Hence for a spherical entangling surface, the above expression shows how the extremal surface begins towards the interior of $V$ as it extends into the bulk geometry. However, if the geometry is such that $K^{i}<0$ on some portion of the entangling surface, then the extremal surface actually extends to $X^{i}>X_{0}^{i}\left(y^{a}\right)$. Clearly, eq. (3.73) does not accommodate this situation where the integration would include contributions from outside of the region $V$ - see section 5 for further discussion.

We can also use the above expansion (3.80) to make an interesting observation about the contributions to $\Delta S$ from near the entangling surface. Let us assume that $K^{i}$ is positive everywhere and then use eq. (3.80) to evaluate $\partial_{i} z$ to leading order in small $z$, or equivalently to leading order in $X^{i}-X_{0}^{i}\left(y^{a}\right)$,

$$
\partial_{i} z=-\frac{d-2}{z}\left(\frac{1}{K^{i}\left(y^{a}\right)}-\frac{\frac{\partial X_{0}^{i}}{\partial y^{b}} \frac{\partial y^{b}}{\partial X^{i}}}{K^{i}\left(y^{a}\right)}\right)+\cdots .
$$

We can choose coordinates $y^{a}$ to coincide with $d-2$ of the coordinates $X^{i}$ at linear order in the vicinity of a point in the boundary, and we call $r$ the remaining $X$ coordinate, orthogonal to the boundary. Substituting into eq. (3.73), we find to leading order

$$
\Delta S=2 \pi \frac{d-2}{d} \int d^{d-1} x K^{-1}\left(T_{00}-T_{r r}\right)+\cdots,
$$

where $K=\sqrt{\sum\left(K^{i}\right)^{2}}$. We have dropped the higher derivative contributions with $T_{\mu \nu}^{(n)}$ in the above expression. Further note the integrand is only well approximated above 
in the vicinity of the entangling surface. Now as we noted above, in the special case of a sphere of radius $R$, we have $K^{r}=+(d-2) / R$. Then the general expression (3.82) reduces to

$$
\Delta S=\frac{2 \pi R}{d} \int d^{d-1} x\left(T_{00}-T_{r r}\right)+\cdots,
$$

which agrees with expanding (3.7) to leading order in $(R-r)$. However, we note that this does not appear a good approximation of $\Delta H$ as given in eq. (3.19), even for small $(R-r)$. This suggests that the infinite derivative expansion in (3.7) is crucial to the ultimate agreement between $\Delta S$ and $\Delta\langle H\rangle$, if we want to introduce localized sources which test the vicinity of the region boundary.

As explained in section (6), one expects quite generally that if $T_{\mu \nu}$ was localized sufficiently close to the entangling surface, then $\Delta\langle H\rangle$ should reduce to that of the Rindler modular Hamiltonian (1.9). Further then, in the regime where $\Delta S=\Delta\langle H\rangle$, one must expect this form to be reflected in the result for $\Delta S$. However, as demonstrated above, this agreement cannot be obtained in our holographic calculations purely by expanding to leading order in $z$ near the boundary, no matter how close and sharply localized near the entangling surface $T_{\mu \nu}$ is. In fact, the more localized $T_{\mu \nu}$ becomes, the more important the higher derivative terms will be, which leads to a significant correction to the leading $z$ term. As concluded above therefore, knowledge of the infrared completion of the bulk minimal surface is always important.

\section{Two-dimensional boundary theories}

For a two-dimensional boundary theory, we can describe a thermal state with the BTZ black hole [46]. However, in this case, the bulk geometry is still locally $\mathrm{AdS}_{3}$ space. Further, in calculations of holographic entanglement entropy, the extremal surfaces are simply geodesics. Combining these two observations, we are able to determine the extremal surfaces analytically and hence we can extend our previous analysis beyond perturbation theory. That is, in contrast with the results in section 2 , in the following we can evaluate $\Delta\langle H\rangle$ and $\Delta S$ for arbitrary values of $R T$. The present analysis also allows us to see the effect of compactifying the AdS boundary and also to check the validity of the inequality (1.8) in a situation where the extremal surface exhibits a 'phase transition.'

Eq. (2.6) already describes the appropriate three-dimensional black hole. However, since we wish to consider the spatial direction as compact, we write the (Euclidean) BTZ metric [46] in more familiar coordinates as

$$
d s_{\mathrm{E}}^{2}=\frac{r^{2}-r_{+}^{2}}{R^{2}} d \tau^{2}+\frac{L^{2} d r^{2}}{r^{2}-r_{+}^{2}}+r^{2} d \phi^{2},
$$


where, as usual, $L$ is the AdS radius and the period of $\phi$ is $2 \pi$. The above geometry is smooth as long as $\tau$ is chosen with period $\beta=2 \pi L R / r_{+}$and so the temperature is given by simply $T=1 / \beta=r_{+} /(2 \pi L R)$. The coordinates in eq. (4.1) are normalized so that the boundary metric is

$$
d s_{\text {boundary }}^{2}=d \tau^{2}+R^{2} d \phi^{2}
$$

Hence the periodicity of the spatial direction is $2 \pi R$ and the boundary is a cylinder with a total area $2 \pi R \beta$. We should note that because the spatial direction is compact, there is a Hawking-Page phase transition [47]. The above black hole geometry is the dominant saddle-point in the gravity path integral for $T>1 /(2 \pi R)$, while for $T<1 /(2 \pi R)$, the dominant saddle-point is simply the thermal $\mathrm{AdS}_{3}$ geometry. We may write the metric for the latter as

$$
d s_{\mathrm{E}}^{2}=\frac{r^{2}+L^{2}}{R^{2}} d \tau^{2}+\frac{L^{2} d r^{2}}{r^{2}+L^{2}}+r^{2} d \phi^{2} .
$$

Implicitly, $\tau$ and $\phi$ are chosen with the same periodicity as in the previous case and the boundary metric is again given by eq. (4.2).

Let us begin with the high temperature phase for which eq. (4.1) describes the correct bulk geometry. It is relatively straightforward to evaluate the entanglement entropy of an interval with an angular width $\Delta \phi$ (and on a constant $\tau$ surface). Of course following eq. (1.1), it is given by the length of the geodesic connecting the endpoints of the interval $V$ on the boundary [17],

$$
S(V)=\frac{c}{3} \log \left[\frac{\beta}{\pi \epsilon} \sinh \left(\frac{\pi R \Delta \phi}{\beta}\right)\right],
$$

where $c=12 \pi L / \ell_{\mathrm{P}}$ is the central charge of the boundary CFT and $\epsilon$ is the shortdistance cut-off in the CFT. ${ }^{12}$

This expression precisely matches the known result previously derived for twodimensional CFT's at finite temperature [2, 48]. However, we should note that this previous result was derived for the case where the spatial direction was noncompact. That is, this same expression (4.4) was derived for any two-dimensional CFT but only in the limit $R \rightarrow \infty$ while holding $\Delta x=R \Delta \phi$ fixed. Hence, we see here that in a holographic $d=2$ CFT, compactifying the spatial direction does not affect this finite temperature entanglement entropy (4.4). Of course, this statement holds when the bulk physics is accurately described by classical Einstein gravity and hence eq. (4.4) only represents the leading contribution in an expansion for large $c$.

\footnotetext{
${ }^{12}$ The latter appears in the holographic calculation by terminating the geodesic at a UV regulator surface positioned at $r=r_{\mathrm{UV}}=L R / \epsilon$ in the bulk geometry.
} 


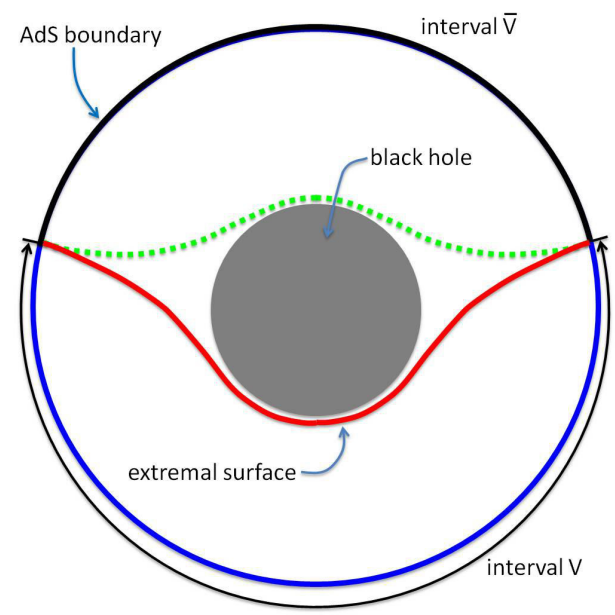

(a)

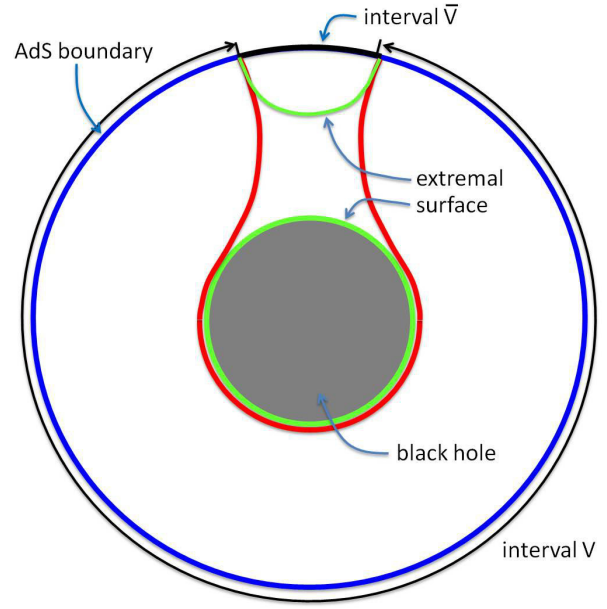

(b)

Figure 1. (Colour Online) Extremal surfaces in the high temperature phase. The figures show a cross-section of the $\mathrm{AdS}_{3}$ black hole at constant $t$. (a) For sufficiently small $\Delta \phi$, the holographic entanglement entropy (1.1) is evaluated with the red geodesic. The dashed green geodesic passing on the other side of the black hole is not homologous to the interval $V$, however, it would yield the entanglement entropy for the complementary interval $\bar{V}$. (b) For large $\Delta \phi$, the dominant saddle-point (in green) has two disconnected components, i.e., the geodesic homologous to $\bar{V}$ and the geodesic wrapping around the horizon.

Implicitly, the above result also assumes that $\Delta \phi$ is sufficiently small. In this high temperature phase, one finds for large enough $\Delta \phi$, that the holographic entanglement entropy experiences a 'phase transition,' as described in figure 1. For any value of $\Delta \phi$, there are two geodesics connecting the endpoints of the interval on the boundary, which pass on either side of the black hole, as shown in figure 1a. However, only one of these (the green geodesic in the figure) is homologous to the boundary interval $V$ and hence this one must be chosen to evaluate the holographic entanglement entropy. The other (the dashed red geodesic) can be used to evaluate the entanglement entropy for the complementary region $\bar{V}$, with the result

$$
S(\bar{V})=\frac{c}{3} \log \left[\frac{\beta}{\pi \epsilon} \sinh \left(\frac{\pi R(2 \pi-\Delta \phi)}{\beta}\right)\right] .
$$

Of course, for $\Delta \phi>\pi$, the latter expression is smaller than $S(V)$ in eq. (4.4). While this geodesic by itself is not homologous to the region of interest, it can be used to construct another extremal surface with two disconnected components, as shown in figure 1b, which is homologous to $V$. The second component consists of a closed (spatial) geodesic which wraps around (the bifurcation surface of) the black hole horizon. The latter 
contributes the standard horizon entropy, i.e.,

$$
S_{\mathrm{BH}}=\frac{2 \pi}{\ell_{\mathrm{P}}} A\left(r_{+}\right)=\frac{2 \pi^{2} r_{+}}{\ell_{\mathrm{P}}}=\frac{2 \pi^{2} c}{3} \frac{R}{\beta} .
$$

Hence combining these results, the entropy for a general interval is given by

$$
S=\frac{c}{3} \min \left[\log \left(\frac{\beta}{\pi \epsilon} \sinh \left(\frac{\pi R \Delta \phi}{\beta}\right)\right), \log \left(\frac{\beta}{\pi \epsilon} \sinh \left(\frac{\pi R(2 \pi-\Delta \phi)}{\beta}\right)\right)+\frac{2 \pi^{2} R}{\beta}\right] .
$$

For general values of $R / \beta$, it would require a numerical evaluation to determine the precise value of $\Delta \phi$ at which there is a phase transition between the two saddle-points occurs. However, in the high temperature limit with $R / \beta \gg 1$, it is straightforward to show that the phase transition occurs at ${ }^{13}$

$$
\Delta \phi \simeq 2 \pi-\log 2 \frac{\beta}{2 \pi R}+\cdots
$$

where the $\cdots$ denotes corrections that are exponentially suppressed by $e^{-2 \pi^{2} R / \beta}$.

Recall in the low temperature phase with $R / \beta<1 /(2 \pi)$, the bulk geometry is simply the thermal $\mathrm{AdS}_{3}$ geometry (4.3). In this case, there is always a single geodesic joining the endpoints of the boundary interval and we have

$$
S=\frac{c}{3} \log \left(\frac{2 R}{\epsilon} \sin (\Delta \phi / 2)\right)
$$

Again this expression precisely matches a known result derived for general two-dimensional CFT's [2, 49]. In this case, this expression (4.9) holds for any two-dimensional CFT but only in the limit $T=0$. Hence, we see here that in a holographic $d=2$ CFT, turning on a small temperature does not affect the entanglement entropy (4.4) to the leading order in the large- $c$ expansion.

Hence comparing the entropy of a low temperature state to that of the vacuum (i.e., $T=0$ ) for a fixed interval, we find $\Delta S=0$. Rather the order $c$ contributions cancel and hence $\Delta S$ is only a quantity of order one. If instead, we compare the entropy of a state in the high temperature phase to that of the vacuum, we find

$$
\begin{aligned}
\Delta S & =\frac{c}{3} \log \left(\frac{1}{2 \pi R T} \frac{\sinh (\pi R T \Delta \phi)}{\sin (\Delta \phi / 2)}\right) \\
& =\frac{\pi^{2}}{18} c\left(R^{2} T^{2}+\frac{1}{4 \pi^{2}}\right) \Delta \phi^{2}+O\left(\Delta \phi^{4}\right) .
\end{aligned}
$$

\footnotetext{
${ }^{13}$ We thank Ian Morrison and Matt Roberts for pointing out an error in the result given here in our original manuscript.
} 
In the first line, we have assumed that $\Delta \phi$ is small enough that the finite temperature entropy is given by eq. (4.4). In the second line, we are expanding the result for $\Delta \phi \ll 1$. Note that the two expressions in eqs. (4.4) and (4.9) are approximately equal in this limit $\Delta \phi \ll 1$ where the effects of compactification and finite temperature can be neglected.

The modular Hamiltonian of a $d$-dimensional CFT for the vacuum on the cylindrical geometry $R \times S^{d-1}$ can be obtained by conformally transforming the result (1.10) for the sphere in Minkowski space [20]. Applying this transformation in the present case with $d=2$, we have

$$
H=2 \pi R^{2} \int_{-\Delta \phi / 2}^{\Delta \phi / 2} d \phi \frac{\cos (\phi)-\cos (\Delta \phi / 2)}{\sin (\Delta \phi / 2)} T_{00} .
$$

In the vacuum, on the cylinder, the energy density is given by $T_{00}=-\frac{c}{24 \pi R^{2}}[50]$. In general at finite temperature, the expression for the energy density will be quite complicated but to leading order in the central charge the energy density does not change until the temperature reaches the high temperature phase $R T>(2 \pi)^{-1}[51]$. In this high temperature phase we have $T_{00}=\frac{\pi}{6} c T^{2}$, which is the standard result for any CFT in the high temperature limit (or in decompactified space) [50].

Combining these results gives

$$
\begin{aligned}
\Delta\langle H\rangle & =\frac{2 \pi^{2} c}{3}\left[1-\frac{\Delta \phi / 2}{\tan (\Delta \phi / 2)}\right]\left(R^{2} T^{2}+\frac{1}{4 \pi^{2}}\right) \\
& =\frac{\pi^{2}}{18} c\left(R^{2} T^{2}+\frac{1}{4 \pi^{2}}\right) \Delta \phi^{2}+O\left(\Delta \phi^{4}\right) .
\end{aligned}
$$

The second line gives an expansion of the result for $\Delta \phi \ll 1$. Comparing with the expansion in eq. (4.10), we see the leading term in both cases agrees and so we saturate the inequality (1.8) for small $\Delta \phi$.

Our results above apply for any value of $\Delta \phi$ and so we may also examine the inequality (1.8) for finite values. Figure 2a shows the difference $\Delta\langle H\rangle-\Delta S$ as a function of $\Delta \phi$ for the high temperature phase. There we see that this difference is positive and increasing for all angles. Hence the inequalities in both eqs. (1.8) and (1.13) are satisfied throughout the full range of $\Delta \phi$. Note the phase transition at large angular sizes, which was discussed above, contributes very little to the slope of the curves. Figure $2 \mathrm{~b}$ shows the ratio $\Delta S / \Delta\langle H\rangle$. This ratio decreases with size and the figure clearly shows that $\Delta S \simeq \Delta\langle H\rangle$ for intervals of small size, as noted above.

\subsection{Thermal Rindler space}

In this section, we consider a two-dimensional CFT in a thermal state in the Rindler wedge. The modular Hamiltonian for this case is given in eq. (1.11). We will use this 


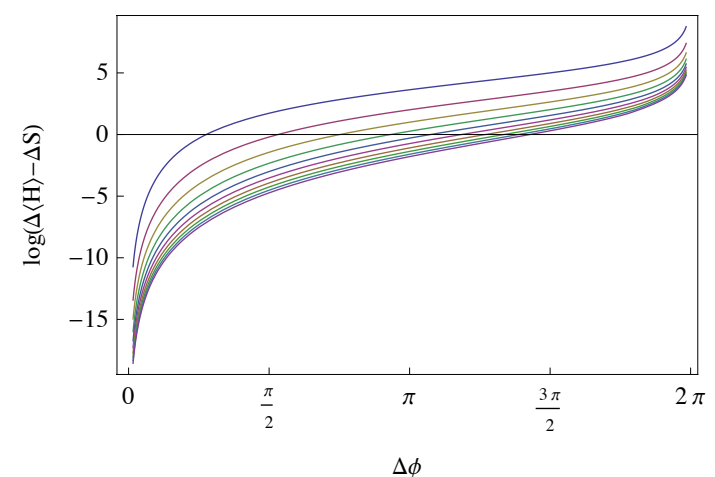

(a)

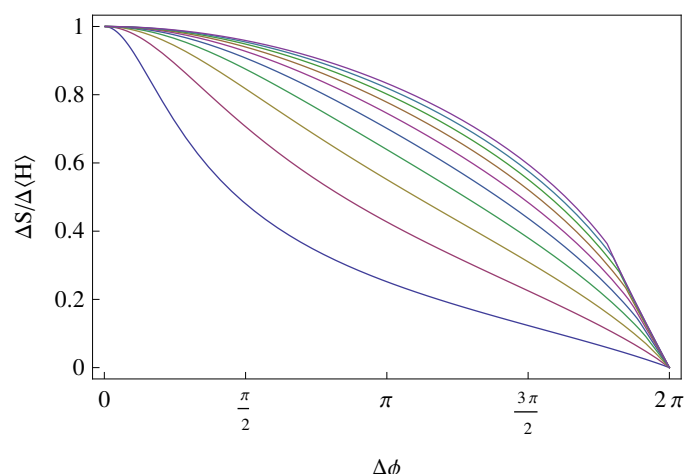

(b)

Figure 2. Comparing $\Delta\langle H\rangle$ and $\Delta S$ in the high temperature phase. Panel (a) shows the $\log$ of the relative entropy and panel (b), the ratio $\Delta S / \Delta\langle H\rangle$, both as functions of angular size $\Delta \phi \in(0,2 \pi)$. The different curves are for $\beta / R=2 \pi \frac{i}{10}$ with $i=1, \ldots, 10$. Curves corresponding to higher temperature (smaller $\beta$ ) have greater relative entropy in (a) and lower ratios $\Delta S / \Delta\langle H\rangle$ in (b).

to compute the relative entropy between states at different temperatures, i.e., both $\rho_{0}$ and $\rho_{1}$ will describe thermal states with temperatures, $T_{0}$ and $T_{1}$, respectively. The expectation value of the stress tensor for both of these states is $T_{00}(x)=\frac{\pi}{6} c T_{i}^{2}$, where $T_{i}$ corresponds to the appropriate temperature. Since Rindler space has infinite volume, we need to introduce a long-distance infrared cut-off $\Lambda$, i.e., we integrate only over $0 \leq x \leq \Lambda$. Given eq. (1.11), we fix the modular Hamiltonian to be $H_{0}=H\left(T=T_{0}\right)$ corresponding to $\rho_{0}$. Then the change of the expectation value of modular Hamiltonian between $\rho_{1}$ and $\rho_{0}$ given by

$$
\Delta\langle H\rangle=\operatorname{Tr}\left(\rho_{1} H_{0}\right)-\operatorname{Tr}\left(\rho_{0} H_{0}\right)=\frac{\pi}{6} c \Lambda\left(\frac{T_{1}^{2}}{T_{0}}-T_{0}\right)-\frac{c}{12}\left(\frac{T_{1}^{2}}{T_{0}^{2}}-1\right) .
$$

Here, we have dropped terms proportional to $\exp \left(-2 \pi T_{0} \Lambda\right)$. The first term on the right hand side is the purely thermal and extensive $(\propto \Lambda)$ contribution, which comes from the large part of the Rindler wedge which is at distances larger than $T^{-1}$ from $x=0$. One can regard the second term as the contribution of the entanglement across the entangling surface $x=0$.

Turning to the holographic calculation of the entanglement entropy, we use the original metric (2.6) with $d=2$ to describe the black hole geometry. The appropriate extremal surface with which to evaluate eq. (1.1) is the geodesic which begins at $x=0$ on the AdS boundary $(z=0)$ and extends out along the event horizon $\left(z=z_{\mathrm{h}}\right)$ at large 
positive $x$. This geodesic is given by

$$
\begin{aligned}
& x(s)=\frac{1}{2} z_{\mathrm{h}} \log \left(4 e^{2 s / L}+1\right), \\
& z(s)=\frac{z_{\mathrm{h}}}{\left(\frac{1}{4} e^{-2 s / L}+1\right)^{1 / 2}},
\end{aligned}
$$

where $s$ is the affine parameter along the geodesic. Note that the geodesic approaches the AdS boundary as $s \rightarrow-\infty$ and extends out along the horizon as $s \rightarrow+\infty$. With $d=2$, eq. (2.9) yields $T=1 /\left(2 \pi z_{\mathrm{h}}\right)$, and we recall that $c=12 \pi L / \ell_{\mathrm{P}}$. Imposing an ultraviolet cut-off $z=\epsilon$ and an infrared cut-off at $x=\Lambda$, the entropy at a generic temperature $T$ becomes

$$
S(T)=\frac{2 \pi}{\ell_{\mathrm{P}}}(s(x=\Lambda)-s(z=\epsilon))=\frac{c}{12} \log \left(\frac{e^{4 \pi T \Lambda}-1}{4 \pi^{2} \epsilon^{2} T^{2}}\right) .
$$

Given this expression, it follows that

$$
\Delta S=S\left(T_{1}\right)-S\left(T_{0}\right)=\frac{\pi}{3} c \Lambda\left(T_{1}-T_{0}\right)-\frac{c}{6} \log \left(\frac{T_{1}}{T_{0}}\right),
$$

where again we are dropping terms that are exponentially small in $\Lambda$.

Combining eqs. (4.14) and (4.17), the relative entropy is

$$
S\left(\rho_{1} \mid \rho_{0}\right)=\Delta\langle H\rangle-\Delta S=\frac{\pi}{6} c \Lambda T_{0}\left(\frac{T_{1}}{T_{0}}-1\right)^{2}+\frac{c}{12}\left(1+2 \log \left(\frac{T_{1}}{T_{0}}\right)-\frac{T_{1}^{2}}{T_{0}^{2}}\right) .
$$

For generic $T_{1}$, this result is always positive because it is dominated by the first term since $\Lambda T_{0,1} \gg 1$. Of course, one must treat the region $T_{1} \sim T_{0}$ more carefully. With $T_{1}=$ $T_{0}$, both $S\left(\rho_{1} \mid \rho_{0}\right)$ and the first derivative $\partial_{T_{1}} S\left(\rho_{1} \mid \rho_{0}\right)$ vanish. The second derivative yields

$$
\partial_{T_{1}}^{2} S\left(\rho_{1} \mid \rho_{0}\right)=\frac{c}{6}\left(2 \pi \frac{\Lambda}{T_{0}}-\frac{1}{T_{0}^{2}}-\frac{1}{T_{1}^{2}}\right) .
$$

This quantity is again positive given $\Lambda T_{0,1} \gg 1$ and so the relative entropy is positive in the vicinity of $T_{1}=T_{0}$. Because of the vanishing first derivative, we also have the equality for small deviations $\delta T=T_{1}-T_{0}$

$$
\Delta S=\Delta\langle H\rangle=c\left(\frac{\pi}{3} \Lambda-\frac{1}{6 T_{0}}\right) \delta T .
$$

In previous calculations, we compared the vacuum state and a thermal state. To compare the thermal state with the vacuum on Rindler space, we can set $T_{1}=0$ and $\Delta\langle H\rangle$ follows from eq. (4.14) as

$$
\Delta\langle H\rangle=-\frac{\pi}{6} c \Lambda T_{0}+\frac{c}{12} .
$$


The vacuum in Rindler space has logarithmic entropy $S \sim c / 6 \log (\Lambda / \epsilon)$. Hence the difference in entropies is

$$
\Delta S=-\frac{\pi}{3} c \Lambda T_{0}+\frac{c}{6} \log \left(\Lambda T_{0}\right)+\mathcal{O}\left(\Lambda^{0}\right) .
$$

Thus the inequality $\Delta\langle H\rangle>\Delta S$ is always valid. Note that there is no meaningful way to say that the relative entropy ${ }^{14}$ approaches zero, i.e., $\Delta\langle H\rangle \rightarrow \Delta S$, for small temperatures since we must keep $\Lambda T_{0} \gg 1$. In fact, the vacuum in Rindler space always remains at an infinite statistical distance from a thermal state since far enough from the origin, i.e., $x \gg 1 / T_{0}$, the thermally excited modes are in presence of a nearly zero Unruh temperature vacuum. This does not happen in comparing the vacuum and a thermal state over a finite interval of size $\ell$. At sufficiently small temperatures, i.e., $T_{0} \lesssim$ $1 / \ell$, the change in the modular Hamiltonian will essentially match the change in the entanglement entropy. In particular, in the previous section, we saw that $\Delta\langle H\rangle$ and $\Delta S$ were always nearly identical for sufficiently small $\Delta \phi$, irrespective of the temperature.

\section{Puzzles about localization}

Most of our previous calculations only probed the asymptotic region in the bulk geometry and in particular, the analysis in section 3 relied heavily on the asymptotic FG expansion. With the latter approach, one can construct the asymptotic geometry for states with an essentially arbitrary expectation value for the stress tensor and other operators. However, one should be aware that in many cases, these expectation values will not correspond to a physical state. In other words, if one really goes beyond the asymptotic expansion to construct the full nonlinear gravity solution, one would find that in many cases, the solution has a naked singularity somewhere in the infrared region. Of course, string theory may be able to resolve some such singularities [52], however, one should expect that most of these singular solutions are simply unphysical. Certainly, our previous analysis does not consider such issues which might arise in defining a global state from imposing a 'smoothness' boundary condition in the infrared. In this section, we consider some apparent paradoxes (and their resolution) which appear from localizing the expectation values which contribute to $\Delta\langle H\rangle$ and $\Delta S$. From this perspective, the relative entropy provides interesting probe of the AdS/CFT correspondence, which reveals constraints on the properties of physical states which would not be easily seen by other means.

\footnotetext{
${ }^{14}$ Note that in evaluating $S\left(\rho_{1} \mid \rho_{0}\right)=\Delta\langle H\rangle-\Delta S, \rho_{1}$ corresponds to the vacuum while $\rho_{0}$ is the thermal state. In our previous calculations, these roles were reversed.
} 


\subsection{Complementary regions in a pure state}

Our general arguments from the previous sections indicate that under the conditions of a small linear perturbation $\delta T_{\mu \nu}$, the inequality in eq. (1.8) is saturated with $\Delta S=\Delta\langle H\rangle$, for a spherical entangling surface. Further it is clear from the holographic calculations that if the perturbation $\delta T_{\mu \nu}$ was completely localized outside of the sphere, it would not change the entanglement entropy, i.e., $\Delta S=0$. Further given the form of the modular Hamiltonian (1.10), it is also clear that for this situation that we also have $\Delta\langle H\rangle=0$. Of course, this is as it must be, since $H_{V}$ is a operator in the algebra generated by local operators in the region $V$, i.e., the interior of the sphere. ${ }^{15}$ The latter would then also extend to more general regions $V$, for which we also expect $\Delta\left\langle H_{V}\right\rangle$ to be given by contributions from the expectation values of operators inside $V$.

Now our first apparent paradox arise from considering instead the case where $\delta T_{\mu \nu}$ is entirely localized inside the sphere. Again we suppose that eq. (1.8) is saturated with

$$
\Delta S_{V}=\Delta\left\langle H_{V}\right\rangle .
$$

The modular Hamiltonian of the vacuum state in the region $\bar{V}$ outside the sphere is given by

$$
H_{\bar{V}}=2 \pi \int_{|x|>R} d^{d-1} x \frac{r^{2}-R^{2}}{2 R} T_{00}(x) .
$$

Our assumption is that the stress tensor vanishes in this complementary region. Hence since $\left\langle T_{00}(x)\right\rangle=0$ in $\bar{V}$, we have

$$
\Delta\left\langle H_{\bar{V}}\right\rangle=0 .
$$

However, if the perturbed state is pure, the entanglement entropy for the two complementary regions, the interior and the exterior of the sphere, must be equal. Holographically, $\Delta S_{V}$ came from the changes in the corresponding extremal surface in the bulk. However, assuming there are no additional horizons in the bulk, as should be the case for a pure state, the same two extremal surfaces (i.e., the one for the vacuum and the one for the perturbed state) also determine $S_{\bar{V}}$. Thus, in this case we have

$$
\Delta S_{V}=\Delta S_{\bar{V}} .
$$

Now combining eqs. (5.1), (5.3) and (5.4), we see $\Delta\left\langle H_{\bar{V}}\right\rangle \neq \Delta S_{\bar{V}}$. In particular then, the equality can not be achieved for $\bar{V}$ no matter how small $\delta T_{\mu \nu}$ is. In fact, assuming we have injected a small positive energy inside the sphere, i.e., $\delta T_{00}>0$, then

\footnotetext{
${ }^{15}$ The full generator of modular flow is $H_{V}-H_{-V}$, while $H_{V}$ is the generator for the modular flow inside $V$.
} 
$\Delta\left\langle H_{V}\right\rangle=\Delta S_{V}=\Delta S_{\bar{V}}>0$. Then we have arrived at a clear contradiction with the positivity of relative entropy since $\Delta\left\langle H_{\bar{V}}\right\rangle-\Delta S_{\bar{V}}<0$. Of course, the resolution to this apparent paradox is that it is not possible to choose to inject (positive) energy only in $V$ and not in $\bar{V}$ for a pure state near the vacuum. There must be enough energy in both $V$ and $\bar{V}$ to ensure the equality of the expectation values of the modular Hamiltonians for the two complementary regions. In the context of the AdS/CFT correspondence, this is a constraint that would not be visible with the FG expansion but that one can imagine arises from global issues in defining a smooth bulk geometry.

We can also make a field theory argument to directly demonstrate this conclusion that the energy of the perturbed state cannot be strictly localized. To see this, we construct the combination

$$
H=H_{V}-H_{\bar{V}} .
$$

This operator generates the conformal transformations which keep the sphere fixed [20]. It annihilates the global vacuum state

$$
H|0\rangle=\left(H_{V}-H_{\bar{V}}\right)|0\rangle=0 .
$$

Now we can write an arbitrary pure state which approaches the vacuum as

$$
|\psi\rangle=|0\rangle+\epsilon|\phi\rangle
$$

with small $\epsilon$. Then using eq. (5.6), we have

$$
\begin{aligned}
\Delta\left\langle H_{V}\right\rangle=\left\langle\psi\left|H_{V}\right| \psi\right\rangle & \simeq \epsilon\left(\left\langle\phi\left|H_{V}\right| 0\right\rangle+\left\langle 0\left|H_{V}\right| \phi\right\rangle\right) \\
& =\epsilon\left(\left\langle\phi\left|H_{\bar{V}}\right| 0\right\rangle+\left\langle 0\left|H_{\bar{V}}\right| \phi\right\rangle\right) \\
& =\Delta\left\langle H_{\bar{V}}\right\rangle .
\end{aligned}
$$

For example then, $|\phi\rangle$ might be generated by creation operators associated to wave packets concentrated inside the sphere. However, the above equality indicates that there is also some energy density built outside the sphere, to linear order in $\epsilon$.

Moreover, the relation (5.6) is completely general, valid for the modular Hamiltonian of any region. To see this note the vacuum state is a pure state belonging to the Hilbert space $\mathcal{H}_{V} \otimes \mathcal{H}_{\bar{V}}$, and hence can be written in a Schmidt decomposition [53]

$$
|0\rangle=\sum_{i} \sqrt{\lambda_{i}}\left|\psi_{i}^{V}\right\rangle \otimes\left|\psi_{i}^{\bar{V}}\right\rangle .
$$

One readily checks doing the partial traces of this state that the $\left|\psi_{i}^{V}\right\rangle$ are the eigenvectors of $\rho_{V}$ and $\left|\psi_{i}^{\bar{V}}\right\rangle$ are those for $\rho_{\bar{V}}$, while $\lambda_{i}$ are the common eigenvalues of both density matrices. Then a simple calculation shows

$$
\left(\rho_{V}\right)^{i \tau} \otimes\left(\rho_{\bar{V}}\right)^{-i \tau}|0\rangle=|0\rangle .
$$


These unitary operators leave the vacuum invariant for any $\tau{ }^{16}$ Expanding for small $\tau$, and taking into account that $\rho_{V} \sim e^{-H_{V}}, \rho_{\bar{V}} \sim e^{-H_{\bar{V}}}$, we obtain (5.6) for any region. In the limit which we are considering, where a perturbed state is approaching the vacuum, there is no way to make a pure state with localized modular energy. This guarantees once $\Delta\left\langle H_{V}\right\rangle=\Delta S_{V}$ we also have $\Delta\left\langle H_{\bar{V}}\right\rangle=\Delta S_{\bar{V}}$ for any region and any pure state in this approximation.

Of course, the latter also represents a restriction that applies for holographic pure states in the AdS/CFT correspondence. However, this observation has a limited utility in general because, as we noted before, the precise form of the modular Hamiltonian is not know except in certain special cases and in general, it is not even local (though it is generated by local fields inside $V$ ). However, in section 6 , we will argue that for holographic CFT's dual to Einstein gravity, expectation values of the modular Hamiltonian for any region, to first order in pure state deviations from the vacuum state, are in fact given by expressions linear in the expectation value of the stress tensor. Hence in this case, the above observation becomes a constraint on the localization of the stress energy in pure states for such a holographic theory.

\subsection{An inequality for $\Delta\langle H\rangle$}

We can use the previous result to relate boundary data in the FG expansion with the formation of horizons or singularities in the infrared region. Suppose as before that we have a global state for which the stress energy inside of a given sphere is small enough that $\Delta S_{V}=\Delta\left\langle H_{V}\right\rangle$. Further, if this global state is pure, it is $S_{V}=S_{\bar{V}}$. However, even if the density matrix describing $\bar{V}$ is not near the vacuum and there is no equality between $\Delta\left\langle H_{\bar{V}}\right\rangle$ and $\Delta S_{\bar{V}}$, we still have from relative entropy in $\bar{V}$ that for a pure state

$$
\Delta\left\langle H_{\bar{V}}\right\rangle \geq \Delta\left\langle H_{V}\right\rangle
$$

for any sphere $V$ with small stress tensor $\left\langle T_{\mu \nu}(x)\right\rangle$. If this inequality is not maintained then either the state is impure or the boundary data does not describe a consistent physical state. In particular, as described above, we expect that the boundary data yields a full gravity solution containing a naked singularity in the infrared region.

Let us consider further the case of an impure state, in which case we expect that the bulk develops a horizon. The discussion in section 4 provides an explicit example of the following considerations. A state near the vacuum inside the sphere $V$ gives again $\Delta S_{V}=\Delta\left\langle H_{V}\right\rangle$. In this situation, we also know that generally $\Delta S_{V} \neq \Delta S_{\bar{V}}$, because for a global impure state the entropies of complementary regions do not coincide. We

\footnotetext{
${ }^{16}$ These unitary operators implement an evolution for an internal time $\tau$. This time flow is called the modular flow [29].
} 
may still ask what is the possible value of $\Delta S_{\bar{V}}$. In the vacuum, the minimal surfaces determining the entanglement entropy for $V$ and $\bar{V}$ coincide, yielding $S_{V}=S_{\bar{V}}$. In the perturbed state, $\Delta S_{V}$ comes from a small variation of this minimal surface. It seems reasonable to expect that the minimal surfaces determining $S_{\bar{V}}$ will contain as one component the same (perturbed) minimal surface. Then this surface would contribute the quantity $\Delta S_{V}$ to $\Delta S_{\bar{V}}$. However, there may also be a horizon contributing positively

some $S_{H}$ to $S_{\bar{V}}$, which of course is not present in the vacuum entropy. In this case, we have $\Delta S_{\bar{V}}=\Delta S_{V}+S_{H}>\Delta S_{V}$. Thus, from the positivity of the relative entropy applied to $\bar{V}$, we again find that the inequality (5.11) is satisfied. It is a logical possibility that the extremal surface determining $S_{\bar{V}}$ does not contain the minimal surface yielding $S_{V}$. In this case, we would expect that there is again a horizon in the interior between the two extremal surfaces preventing one from collapsing to the other. We think this possibility is not probable if we are in the regime where $\Delta S_{V}=\Delta\left\langle H_{V}\right\rangle$. The area of any such putative minimal surface would be very large compared to the two component surface comprised of the horizon and the surface in the asymptotic region near $V$. However, even in this situation, the area of the minimal surface determining $S_{\bar{V}}$ would be much larger than that for $S_{V}$ and eq. (5.11) would still hold.

In conclusion, it seems that inequality (5.11) cannot be violated even for impure states. Hence violations of this inequality should signal that the boundary data appearing in the FG expansion does not correspond to a physical state. Recall that the modular Hamiltonian for the interior and exterior of the sphere are explicitly given in eqs. (1.10) and (5.2). Hence it is straightforward to explicitly evaluate $\Delta\langle H\rangle$ on both sides of eq. (5.11) and test this inequality.

It would be interesting to have a purely QFT understanding on why $\left\langle T_{\mu \nu}\right\rangle$ not satisfying this inequality is unphysical. Returning the QFT discussion above, eq. (5.8) need not apply in general because the order $\epsilon^{2}$ terms are important in $\bar{V}$. Instead one would have $\Delta\left\langle H_{\bar{V}}\right\rangle=\Delta\left\langle H_{V}\right\rangle+\epsilon^{2}\left\langle\phi\left|H_{\bar{V}}\right| \phi\right\rangle$ and hence eq. (5.11) demands that $\left\langle\phi\left|H_{\bar{V}}\right| \phi\right\rangle \geq 0$. Examining $H_{\bar{V}}$ in eq. (5.2), this inequality seems to indicate that CFT states in Minkowski space cannot support a negative energy density over large regions, which certainly seems an intuitive conclusion.

\subsection{Annular regions}

Consider now an annular region $A$ bounded by two concentric spheres with radii $R_{1}<$ $R_{2}$. We denote the regions within the two spheres as $V_{1}$ and $V_{2}$. In the holographic context, depending on the ratio $R_{2} / R_{1}$ and the dimension $d$, the minimal surface can have two different topologies. In one regime where $R_{2} \sim R_{1}$, the minimal surface has the shape of a half torus connecting the two spheres in the asymptotic boundary. In the opposite regime where $R_{2} \gg R_{1}$, the surface is formed by two separate spherical 

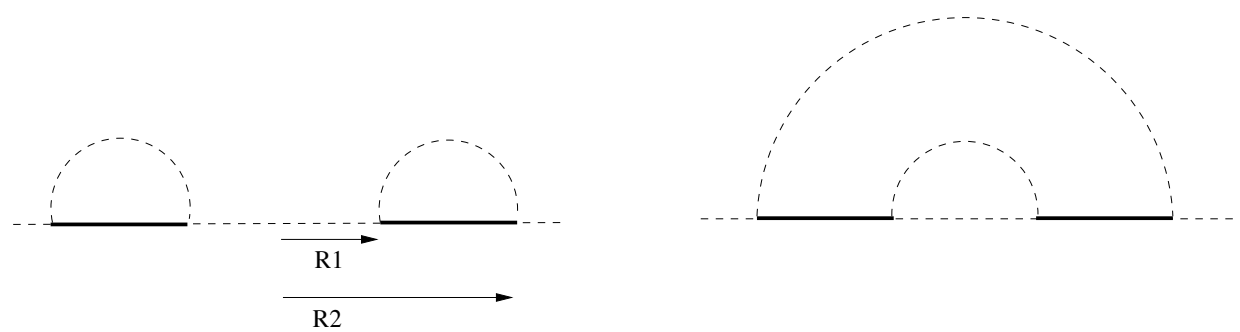

Figure 3. The annular region on the AdS boundary is shown with the two solid lines. When the radius $R_{1}$ and $R_{2}$ of the annulus approach each other the minimal surface has the shape of a half torus connecting the two spheres (left panel). When $R_{2} / R_{1}$ is greater than a certain value the minimal surface is formed by the two spherical caps ending at the spheres of radius $R_{1}$ and $R_{2}$ at the boundary (right pannel).

caps, each one attached to one of the spheres on the boundary ${ }^{17}$ - see figure 3 . We focus on the latter regime in the following.

Now if we turn on a small expectation value for $\left\langle T_{\mu \nu}\right\rangle$ using the FG expansion, we obtain a variation $\Delta S_{A}$ for the annulus which is linear in $\left\langle T_{\mu \nu}\right\rangle$, and according to the general arguments above, this variation will equal $\Delta\left\langle H_{A}\right\rangle$. However, we note that $\Delta\langle H\rangle$ is the expectation value of an operator with support entirely inside the annular region.

For the phase where the minimal surface has two disconnected components, one attached to each spherical boundary of the annular region, we know the contribution to $\Delta\langle H\rangle$ for any linear $\left\langle T_{\mu \nu}\right\rangle$ exactly. The contribution from each cap can be evaluated independently, and as shown in section 3.1, each of these contributions satisfies $\Delta\langle H\rangle=$ $\Delta S$. Hence we find

$$
\Delta\left\langle H_{A}\right\rangle=\int_{|x|<R_{1}} d^{d-1} x \frac{R_{1}^{2}-r^{2}}{2 R_{1}} T_{00}(x)+\int_{|x|<R_{2}} d^{d-1} x \frac{R_{2}^{2}-r^{2}}{2 R_{2}} T_{00}(x) .
$$

Clearly the expression on the right-hand side includes contributions of $T_{\mu \nu}(x)$ from outside of $A$, i.e., from $x<R_{1}$, inside the smaller sphere. While we do not have a precise expression for $H_{A}$ at this point, we re-iterate that it only has support inside of $A$. As such, eq. (5.12) becomes a nonlocal constraint on small stress tensor perturbations of physical states in the holographic framework.

\footnotetext{
${ }^{17}$ Note that the inner sphere of radius $R_{1}$ provides an example where a portion of the entangling surface has $K^{i}<0$ and so, as discussed below eq. (3.80), the extremal surface in the bulk bends away from the interior of $A$. In fact, this behaviour also persists in the regime where $R_{2} \sim R_{1}$ and the minimal surface has the topology of a half torus, as observed in [54].
} 
We might also consider the exterior of the annulus, $\bar{A}=V_{1} \cup \bar{V}_{2}$. Working again in the regime with $R_{2} \gg R_{1}$, holographic entanglement entropy is again determined by the area of the two spherical caps in the bulk. In this case, we would have

$$
\Delta\left\langle H_{\bar{A}}\right\rangle=\int_{|x|<R_{1}} d^{d-1} x \frac{R_{1}^{2}-r^{2}}{2 R_{1}} T_{00}(x)+\int_{|x|>R_{2}} d^{d-1} x \frac{r^{2}-R_{2}^{2}}{2 R_{2}} T_{00}(x),
$$

where the contributions come entirely from the region external to the annulus or alternatively from within $\bar{A}$. Hence one might guess that $H_{\bar{A}}=H_{V_{1}}+H_{\bar{V}_{2}}$. In particular, this structure would yield a density matrix with the product form $\rho_{\bar{A}}=\rho_{V_{1}} \otimes \rho_{\bar{V}_{2}}$.

\section{Discussion}

In this paper, we have examined relative entropy for some particular states and entangling surfaces in the context of the AdS/CFT correspondence using the standard prescription for holographic entanglement entropy (1.1). Our results here constitute a strong test of this holographic entropy formula. A notable case is the sphere for which we have shown in section 3.1 by direct calculation that holographic entanglement entropy yields the correct entropy for any perturbation of the vacuum, to linear order.

It is remarkable the inequality (1.8) expressing the positivity of the relative entropy, is in fact saturated at leading order and so this equality provides an equation that any first order deviations of holographic entropy must satisfy. The equality $\Delta S=\Delta\langle H\rangle$ then becomes an interesting tool. In fact, we can think of reversing the logic of our tests and trying to obtain information about the modular Hamiltonian, or equivalently the reduced density matrix, from the holographic entanglement entropy. In this sense, the entanglement entropy has the potential to provide a full 'vacuum state tomography.' Let us recall that any pure perturbation of the vacuum can be written as

$$
|\psi\rangle=|0\rangle+\epsilon|\phi\rangle
$$

with some small $\epsilon$. Then the expected change in the entropy and modular energy are

$$
\Delta S=\Delta\left\langle H_{V}\right\rangle=\epsilon\left(\left\langle 0\left|H_{V}\right| \phi\right\rangle+\left\langle\phi\left|H_{V}\right| 0\right\rangle\right)
$$

The knowledge of $\Delta S$ for any perturbation gives us the expectation values on the right hand side. The knowledge of these expectation values for any $|\phi\rangle$ and the fact that $H_{V}$ is an operator localized in the region $V$ imply we can in principle reconstruct the full density matrix from the entropy functional. To see this let us recall the expression for 
the Schmidt decomposition of the vacuum: $|0\rangle=\sum_{i} \sqrt{\lambda_{i}}\left|\psi_{i}^{V}\right\rangle \otimes\left|\psi_{i}^{\bar{V}}\right\rangle$. In this basis, an arbitrary global pure state writes

$$
|\phi\rangle=\sum_{i, j} \beta_{i j}\left|\psi_{i}^{V}\right\rangle \otimes\left|\psi_{j}^{\bar{V}}\right\rangle
$$

Writing $\rho_{V}^{0}=e^{-H_{V}}$, with the particular normalization $\operatorname{Tr}\left[e^{-H_{V}}\right]=1$, the modular Hamiltonian is simply

$$
H_{V}=\sum_{i}-\log \left(\lambda_{i}\right)\left|\psi_{i}^{V}\right\rangle\left\langle\psi_{i}^{V}\right|
$$

Then, after a little algebra, eq. (6.2) gives

$$
\Delta S=\epsilon \operatorname{Tr}\left(\left(\beta+\beta^{\dagger}\right) H_{V} e^{-H_{V} / 2}\right)
$$

If $\Delta S$ is known for any $\phi$, represented by the arbitrary matrix $\beta$ in this equation, we can obtain the matrix $H_{V} e^{-H_{V} / 2}$ as a solution of a set of linear equations. ${ }^{18}$ In other words, there is a unique operator in $V$ such that all linear entropy perturbations for pure state deformations coincide with the value of $\Delta\left\langle H_{V}\right\rangle$.

In principle, this idea allows us to reconstruct the full density matrix of a region based only in the entanglement entropy functional. In particular then, in the context of the AdS/CFT correspondence, it seems that the latter is readily accessible using the standard holographic prescription (1.1). For example, based on our results in section 3, we can reconstruct the full modular Hamiltonian operator for the vacuum reduced to the region within a sphere and the result coincides precisely with the standard expression (1.10) for a CFT. In order to show this, we note we are doing an experiment devised to produce pure deviations of the vacuum as in eq. (6.1) in the boundary theory. In the AdS/CFT context, this excitation is translated to the bulk language by the effect which it has on expectation values of operators. We can say the excitation will be defined by a series of expectation values for certain operators on the boundary which are turned on linearly in $\epsilon$,

$$
\Delta\langle\mathcal{O}\rangle \simeq\langle\psi|\mathcal{O}| \psi\rangle-\langle 0|\mathcal{O}| 0\rangle=\epsilon(\langle\phi|\mathcal{O}| 0\rangle+\langle 0|\mathcal{O}| \phi\rangle)
$$

Hence, the reasoning which leads to the vacuum state tomography for the sphere is given by the following steps:

\footnotetext{
${ }^{18}$ Here all the eigenvalues $\lambda_{i}$ are assumed to be different from zero, or equivalently the density matrix $\rho_{V}$ has inverse. Otherwise the modular Hamiltonian has infinite coefficient for $\left|\psi_{i}^{V}\right\rangle\left\langle\psi_{i}^{V}\right|$, and hence is undefined under finite additions of this projector. This ambiguity for $H_{V}$ is seen clearly from 6.2 since in this case $\left\langle\phi_{i}^{V} \mid 0\right\rangle=0$ and then additions of $\left|\psi_{i}^{V}\right\rangle\left\langle\psi_{i}^{V}\right|$ in $H_{V}$ do not change $\Delta S$. In QFT these local excitations in $V$ completely orthogonal to the vaccum are not allowed as a consequence of the Reeh-Slieder theorem [55].
} 
1. Since the holographic prescription (1.1) yields the entanglement entropy purely in terms of the geometry, linear (order $\epsilon$ ) perturbations in the entropy should depend only on linear (order $\epsilon$ ) perturbations of the bulk metric.

2. Linear perturbations in the bulk metric can be separated as having two different origins. The first one is due to order $\epsilon$ terms in the boundary data giving the expectation value of the stress tensor, which modifies the boundary conditions. The second one is due to perturbations of the bulk stress tensor which are also linear in $\epsilon$. The latter modify the source of Einstein equations.

3. The bulk stress tensor gets corrections from deviations of bulk matter fields and these are in turn related to the boundary data for expectation values of dual operators. However, the bulk stress tensor is quadratic in the matter fields and so does not yield corrections linear in $\epsilon$ in the vacuum. ${ }^{19}$ Alternatively, one can argue the absence of order $\epsilon$ corrections because if they did exist, changing the sign of $\epsilon$ would lead to an unphysical bulk stress tensor, i.e., not satisfying the null energy conditions.

4. Hence, correction to $S$ linear in $\epsilon$ can only depend on the linear perturbations of the boundary stress tensor for the minimal surface of any region.

5. For the case of the sphere, we have shown the linear terms on $\Delta S$ coincide with the ones in the expectation value of the operator $H=2 \pi \int d x^{d-1} \frac{R^{2}-r^{2}}{2 R} T_{00}(x)$.

6. This operator $H$ is localized inside the sphere (i.e., belongs to the algebra of operators generated by local fields on the sphere). Hence, it is the unique operator in the sphere which does the job of satisfying the equation $\Delta\langle H\rangle=\Delta S$ for any pure deviation of the vacuum to linear order in $\epsilon$. Hence it is the modular Hamiltonian of the sphere.

It is interesting to see what obstacles arise to reconstructing the modular Hamiltonian for other regions. As above, we have that for a general region $\Delta S$ is linear in $T_{\mu \nu}(x)$ to first order in $\epsilon$. However, here we find two related problems. First consider the case of a minimal surface corresponding to a region $V$ which in terms of the FG coordinate $z$ is single-valued, that is, for any $x \in V$ we have a unique $z(x)$ describing

\footnotetext{
${ }^{19}$ We stress that this is only for variations around the vacuum, where the bulk stress tensor is given by the cosmological constant alone. For other states, it is clear there will be linear terms in expectation values of other operators, i.e., . charge density operator for a state with non zero chemical potential. The argument fails in this case because the change of order $\epsilon$ in the stress tensor is infinitesimal with respect to the stress tensor for $\epsilon=0$, which is non zero for non vacuum states. Hence we can change the sign of $\epsilon$ without implying a failure of null energy condition.
} 
the surface (i.e., the one corresponding to an ellipsoidal region with $K^{i}>0$ everywhere along the entangling surface). Using the FG expansion, the contribution to $\Delta S$ in eq. (3.73) involves time and spatial derivatives of $T_{\mu \nu}(x)$ of arbitrarily high order. Even if the spatial derivatives can be eliminated by integration by parts, the time derivatives remain. In section 3.1, it was a surprising result that for the sphere these time derivatives finally disappear from the final expression. However, the result for a general surface cannot be considered as the expectation value of an operator localized in $V$, because even if it depends only of $T_{\mu \nu}(x)$ for $x \in V$, it depends on arbitrary derivatives of the stress tensor. For a minimal surface extending outside $V$, such as the annulus discussed in section 5 , this nonlocality of the contribution is seen more directly since $\Delta S$ involves $T_{\mu \nu}$ outside $V$. In fact, these two types of nonlocality can be put on the same basis by writing the variation of the metric tensor in terms of the boundary-tobulk Green's function in coordinate representation. That is, we can write eqs. (3.5) and (3.15) as

$$
\delta g_{\mu \nu}(y, z)=\int d^{d} x G(x-y, z) T_{\mu \nu}(x),
$$

where $G(x-y, z)$ is proportional to the Fourier transform of the Green's function of section 3.1 in momentum space, ${ }^{20}$

$$
G(x-y, z)=\frac{l_{p}^{d-1}}{d L^{d-1} 2^{d / 2-1} \Gamma[d / 2+1]} \int \frac{d^{d} p}{(2 \pi)^{d}} \theta\left(-p^{2}\right) \frac{z^{d / 2}}{p^{d / 2}} J_{d / 2}(|p| z) e^{-i p x} .
$$

Hence, generically, because this Green's function is not of compact support, the contribution of $T_{\mu \nu}(x)$ for any spacetime point $x$ will not vanish in the variation of the area for a given minimal surface. This is so unless some conspiracy between the particular minimal surface and the tracelessness and conservation of $T_{\mu \nu}$ occurs. This is the case of the sphere, where the contribution is localized inside $V$, but we do not expect the latter property to extend to the case of general surfaces.

The question is how is this possible. The expectation value of the modular Hamiltonian should be localized in $V$. The answer to this apparent contradictions has to reside in the fact that the expression of the result for $\Delta S$ in terms of operator expectation values suffer from two different types of ambiguities. First, we do not have full control on which perturbations for $T_{\mu \nu}$ are generated by genuine pure deviations from the vacuum, as discussed in section 5. Some of the constraints we know, for example, the expectation value of $T_{\mu \nu}$ has to satisfy $\Delta\left\langle H_{V}\right\rangle=\Delta\left\langle H_{\bar{V}}\right\rangle$ for any sphere. The same equality holds for the (unknown) modular Hamiltonian for any general region. The second source of ambiguities is due to the fact that since operators obey time evolution

\footnotetext{
${ }^{20}$ The integration is over time-like momentum since only time-like momentum appears in the contributions $\left\langle 0\left|T_{\mu \nu}(x)\right| \psi\right\rangle+\left\langle\psi\left|T_{\mu \nu}(x)\right| 0\right\rangle$ because physical states have momentum inside the light cone.
} 
laws the expectation values at different times could in principle be rewritten as expectation values for other operators at a single time. Hence, is its natural to suppose that the expression of $\Delta S$ for a general surface, given in terms of $T_{\mu \nu}$ in all spacetime, could be converted into one of some other operator inside $V$ once these constraints are fully understood.

For the case of two (or more) well separated spheres $A$ and $B$, where the minimal surface consists of the separate minimal surfaces for the spheres, it is evident $\Delta S$ is also the sum of $\Delta S$ for both spheres separately. In this case, one has that the modular Hamiltonian can be reconstructed again, and coincides with the sum of the those for separated spheres, because $\Delta S$ depends on $T_{\mu \nu}$ inside the region $A \cup B$ only. This is consistent with the mutual information $I(A, B)=S(A)+S(B)-S(A \cup B)$ being zero in this case. Mutual information is an upper bound to connected correlators, and if it is zero it means correlators of operators in $A$ and $B$ factor out, to leading order in large $N$ (or large central charge). ${ }^{21}$

It is intriguing that Renyi entropies [22, 23] for these same configurations do not separate into the contributions of $A$ and $B$ to this same order in $N$, for $d=2$ $[18,25]$ or higher dimensions [56]. However, recall that Renyi entropies are given by $S_{n}=(1-n)^{-1} \log \left[\operatorname{tr} \rho^{n}\right]$ and so depend on powers of the density matrix. These powers are very different from the density matrix itself, as well as, very different from any finite energy density state in the region. In contrast, the entanglement entropy is the limit $n \rightarrow 1$ and hence only feels states near to $\rho$. Let us look at a simpler case which, while it is quite different to the specific situation we are considering above, still exemplifies the relevant ideas. Hence we think of a global thermal state with $\rho=e^{-\mathcal{H} / T} / Z$ where $\mathcal{H}$ is the standard Hamiltonian. Then Renyi entropies are quantities related to states at different temperatures. Now it is always possible to have a phase transition at some critical temperature where the $N$ dependence of various physical quantities changes, e.g., section 4 described an example where the energy density suffers a phase transition with different dependence on $N .^{22}$ It follows then that the corresponding Renyi entropies exhibit the same phase transition, since changing the Renyi order $n$

\footnotetext{
${ }^{21}$ It is worthwhile to note that our discussion of the reconstruction of the modular Hamiltonian for holographic theories relies on the geometric prescription (1.1) to calculate the entanglement entropy. Of course, this formula is only expected to yield the leading contribution in an expansion of large central charge. In principle, this limitation represents another obstacle to recovering the full modular Hamiltonian.

${ }^{22}$ In the case of two decoupled regions in $d=2$, Renyi entropies for integer $n>1$ do not decouple. This corresponds to lower "internal" temperature for the region. Hence, the phase transition is better described as a screening phase transition, where the entropy, corresponding to a state of higher internal temperature (the vacuum state in $A \cup B$ here), does not see correlations, while Renyi entropies detect these correlations at lower internal temperatures. We owe this observation to Hong Liu.
} 
and the temperature $T$ are the same thing in this case. However, we can also expect similar behaviour for the Renyi entropies in a more general context. In particular, our discussion above indicates that the effective modular Hamiltonian, which is the one relevant for computation of correlation functions, decouples for the two well separated spheres. It does not include all information on higher order $N$ corrections, which must still play an important role in determining the Renyi entropies.

While in general the modular Hamiltonian for a generic region is not a local operator, one expects that very close to the entangling surface of any region $V, H_{V}$ will approach the simple local form given in eq. (1.9) for Rindler space. We readily see this behaviour in eq. (1.10) as we approach $r \sim R$ for the spherical entangling surface. However, the local Rindler expression should be the leading contribution in the modular Hamiltonian independent of the shape of the surface. One approach [57] to understanding this general result is this Rindler term provides short distance part of $\rho_{V}$ that encodes the correlators in the vicinity of the boundary of the causal domain defined by the entangling surface and in the UV, these correlators have the same structure as in flat space, i.e., in the vicinity of a Rindler horizon. Alternatively, as alluded to in various points in our discussion, one can think of the Rindler Hamiltonian as defining a thermal density matrix with a local effective temperature of $T_{\text {eff }}=1 /(2 \pi x)$ where $x$ is the (orthogonal) distance to the entangling surface. Hence as we approach this boundary, the effective temperature diverges and this Rindler term overwhelms any other fixed contributions to the density matrix. Hence along the lines of our discussion of vacuum state tomography, we might attempt to verify the appearance of a Rindler-like contribution in the present holographic setting. In particular, we are thinking here of evaluating $\Delta S$ for perturbations localized near the boundary of the region. This independence of shape for the contribution of these localized sources should then be associated to the surface being minimal — remember in this calculation the contribution would depend on a localized $\delta g_{\mu \nu}$ near the boundary and the dependence on the variation of the surface shape far from the source would not contribute precisely because it is minimal. However, the results of section 3.4 suggest that to have a sufficiently localized $\delta g_{\mu \nu}$ we might need to choose a gauge for the boundary-to-bulk Green's function which is different from the one in eq. (6.8). This reasoning would then lead us closer to a purely thermodynamic understanding of the standard prescription (1.1) for holographic entanglement entropy.

In our previous discussion, we argued that the expectation values of operators other than $T_{\mu \nu}$ only appear quadratically in $\Delta S$ and one form of this argument relies on the null energy condition of the bulk stress tensor. Further, these quadratic order contributions must be negative in order to preserve the positivity of the relative entropy. It seems natural the sign of these contributions could be directly related to the null 
energy condition. In fact, for the sake of the argument, we can think directly in terms of the change of entropy due to the bulk stress tensor perturbation. This encodes all the information from the expectation value of fields at the boundary which is relevant for the calculation of $\Delta S$. The variation of the metric due to perturbations on the bulk stress tensor can be written with an expression similar to eq. (6.7), but where now the integration is over bulk spacetime, the boundary stress tensor is replaced by the perturbation of the bulk stress tensor, and the Green's function is the bulk-to-bulk Green's function [58]. It would be interesting to find out if null energy condition alone can ensure a definite sign for this contribution of $\delta g_{\mu \nu}$ to the change in the area of the minimal surface in a general situation. In other words, the area of the minimal surface of any boundary region $V$ in presence of a bulk $T_{\mu \nu}$ satisfying the null energy condition has to be smaller than the one corresponding to $V$ in pure AdS spacetime.

Bekenstein argued that all systems must satisfy an inequality of the form [59]

$$
S \leq 2 \pi R E
$$

where $S$ and $E$ are the entropy and energy of a system confined to a region of size $R$ see appendix A.4. While this bound was originally derived with a thought experiment involving dropping an object into a black hole, eq. (6.9) does not involve Newton's constant and so it should be possible to understand the bound entirely in terms of flat space physics. Unfortunately, as presented, all of the physical quantities in eq. (6.9) are ambiguous. However, these ambiguities can be eliminated by re-interpreting the bound in terms of the inequality (1.8) expressing the positivity of the relative entropy $[60,61]$ i.e., $\Delta S \leq \Delta\langle H\rangle$. As described in the appendix, one can apply eq. (1.8) in Bekenstein's thought experiment where the region of interest is Rindler space and the result is precisely the inequality in eq. (6.9). Relating eq. (6.9) to relative entropy makes clear that the physics behind the Bekenstein bound is simply quantum mechanics and special relativity.

Of course, to make progress with this approach, we must know the modular Hamiltonian for a given situation. Therefore, let us turn to the example of a spherical entangling surface for a CFT in which case the modular Hamiltonian is given by eq. (1.10). One observation is that if the bound is expressed in terms of the total energy enclosed, as in eq. (6.9), then the precise bound depends very much on how the energy is deposited within the sphere. For example, for a smooth distribution of energy, analogous to those considered in section (2), one finds $\Delta S \leq \frac{2 \pi}{d+1} R E$ while if the energy is localized near the center of the ball enclosed by the sphere $\Delta S \leq \pi R E$. Both of these inequalities have the same form as that in eq. (6.9) and only the overall numerical factor changes on the right-hand side. A more dramatic change arises if the energy is deposited in a spherical shell of roughly radius $R$ and width $w$ with $w \ll R$. In this 
case, eq. (1.8) becomes $\Delta S \leq 2 \pi w E$ and so the relevant length scale that emerges here is, in fact, the width of the shell. This behaviour is reminiscent of the result in [62], where it was argued that the Bekenstein bound is controlled by the shortest dimension (rather than the largest) for matter confined to an elongated region. Of course, the discussion there relied on considerations of how the weakly gravitating matter focussed light rays passing through the region. A similar result can be inferred from our holographic calculations for the strip geometry in section 3.4. To linear order where eq. (1.8) is saturated, we find for a smooth energy distribution that $\Delta\langle H\rangle \propto \ell E$ where $\ell$ is the width of the strip. Hence again it appears that the shortest distance sets the geometric scale for the Bekenstein bound.

Of course, the example of the strip reminds us that in general the 'modular energy' in eq. (1.8) can be quite dissimilar to the energy appearing in eq. (6.9). Our holographic result for $\Delta S$ in eq. (3.78) shows that the pressure $T_{x x}$ appears on a more or less equal footing with the energy density $T_{00}$. Hence using the saturation of eq. (1.8), we expect for homogenous (CFT) matter distributions that the bound will be set by

$$
\Delta\langle H\rangle \simeq \ell V\left[\frac{d+1}{d-1} T_{00}-T_{x x}\right],
$$

where $V=B^{d-2} \ell$ is the volume of the strip. The resulting bound is qualitatively different from the Bekenstein bound in eq. (6.9) since we can not expect the quantity in eq. (6.10) to be proportional to the energy in the strip. In principle, for quantum matter, $T_{00}$ and $T_{x x}$ do not need to satisfy any relation and are not even constrained by classical energy conditions. So the bound set by eq. (6.10) can be much more (or less) constraining than a bound set by $T_{00}$ alone. In particular, if one could realize $T_{x x} \simeq \frac{d+1}{d-1} T_{00}$, we would have the interesting conclusion that $\Delta S \leq 0$, i.e., the entropy in the perturbed state has to be smaller than that in the vacuum state. Of course, these results are symptomatic of the fact that in general, the modular Hamiltonian will contain contributions involving operators other than the energy density and in fact, operators unrelated to the stress-energy tensor. Hence the bounds set by eq. (1.8) will generically be far more complicated than the simple expression appearing in eq. (6.9). Further we must add that although the interpretation of the Bekenstein bound in terms of eq. (1.8) gives a general prescription which is free of ambiguities, unfortunately, without a clear understanding of the modular Hamiltonian for a given situation, this interpretation is left somewhat lacking.

Some recent references $[38,63]$ also consider relations similar to the first law of thermodynamics, i.e., $d E=T d S$, for entanglement entropy — see also [64, 65]. In particular, the discussion in [38] centers on the proportionality between the energy within a small region and the entanglement entropy of the same region, which is seen 
in several examples. In the present paper, we have seen the origin of this proportionality is the equation $\Delta\langle H\rangle=\Delta S$. However, we must again remark that it is in general a different 'type' of energy, the modular 'energy,' that enters into a proper definition of the equation. For example, if this is not taken into account, the proportionality factor between energy and entropy for a spherical entangling surface depends on the distribution of the energy inside it — as was already observed in [38]. For more general (i.e., non-spherical) geometries, $\Delta S$ is simply not proportional to the energy, but rather other operators will appear in the modular Hamiltonian and in the expression for $\Delta\langle H\rangle$.

\section{Acknowledgements:}

We would like to thank Eugenio Bianchi, Patrick Hayden, Marina Huerta, Andreas Karch, Hong Liu, Masahiro Nozaki and Rafael Sorkin for discussions. Research at Perimeter Institute is supported by the Government of Canada through Industry Canada and by the Province of Ontario through the Ministry of Research \& Innovation. RCM also acknowledges support from an NSERC Discovery grant and funding from the Canadian Institute for Advanced Research. Work by DDB and HC was supported by CONICET, Universidad Nacional de Cuyo, and CNEA, Argentina. LYH is supported by the Croucher Foundation.

\section{A Comments on Relative Entropy}

Relative entropy provides a precise measure of the statistical distance between two states. Given a state $\rho_{1}$, the probability of confounding it with $\rho_{0}$ after $n$ trials of some measurement is asymptotically exponentially decreasing for large $n$ as

$$
e^{-n S\left(\rho_{1} \mid \rho_{0}\right)}
$$

In this sense. relative entropy is commonly thought as a measure of the distinguishability between states [66].

As mentioned, relative entropy is positive and increasing with system size,

$$
\begin{aligned}
& S\left(\rho_{1}^{V} \mid \rho_{0}^{V}\right) \geq 0 \\
& S\left(\rho_{1}^{V} \mid \rho_{0}^{V}\right) \leq S\left(\rho_{1}^{W} \mid \rho_{0}^{W}\right), \quad V \subseteq W .
\end{aligned}
$$

The monotonicity property (A.3) is a particular case of monotonicity under general completely positive trace preserving maps (CPTP). These are linear maps of density matrices in one space into density matrices in another one, which are physical in the 
sense they are combinations of operations such as unitary evolution, partial tracing and enlarging the system with a new subsystem. The general expression of a CPTP map is $[53]$

$$
\rho^{\prime}=\sum_{i} M_{i} \rho M_{i}^{\dagger}, \quad \sum_{i} M_{i}^{\dagger} M_{i}=I
$$

for matrices $M_{i}$ with arbitrary dimension, i.e., not necessarily square matrices. Then, more generally the relative entropy satisfies

$$
S\left(\rho_{1} \mid \rho_{0}\right) \geq S\left(\rho_{1}^{\prime} \mid \rho_{0}^{\prime}\right)
$$

The partial trace over a subsystem as in (A.3) is one example of CPTP map. Such CPTP maps then generally entail the loss of distinguishability between states.

\section{A.1 $\Delta S=\Delta\langle H\rangle$ for first order perturbations}

Recall that the relative entropy only vanishes for identical states. Here we expand on the discussion around eq. (1.12) to see what to expect for the relative entropy of nearby states. Keeping our reference state $\rho_{0}$ fixed, we move through a family of states $\rho_{1}(\lambda)$ with a parameter $\lambda$ such that $\rho_{1}(\lambda=0)=\rho_{0}$, i.e., the states coincide for $\lambda=0$. Hence we have that $S\left(\rho_{1}(0) \mid \rho_{2}\right)=0$ but $S\left(\rho_{1}(\lambda) \mid \rho_{2}\right)>0$ for both $\lambda>0$ and $\lambda<0$. Therefore assuming that $S\left(\rho_{1}(\lambda) \mid \rho_{2}\right)$ describes a smooth curve, it must have zero first derivative at $\lambda=0$. This then implies

$$
\Delta S=\Delta\langle H\rangle
$$

to first order in $\lambda$ at $\lambda=0$. For nearby thermal equilibrium states, this relation is just the well known thermodynamic equation $\Delta S=\Delta E / T$.

Another way to see the above equality is to evaluate the first order perturbation of $S(\rho)$ and $H(\rho)$ for a density matrix

$$
\rho=\frac{e^{-(H+\delta H)}}{\operatorname{tr}\left(e^{-(H+\delta H)}\right)}
$$

Then to linear order in $\delta H$, we have that both coincide with

$$
\Delta S=\Delta\langle H\rangle=\frac{\operatorname{tr}\left(e^{-H} H\right) \operatorname{tr}\left(e^{-H} \delta H\right)}{\left(\operatorname{tr}\left(e^{-H}\right)\right)^{2}}-\frac{\operatorname{tr}\left(e^{-H} H \delta H\right)}{\operatorname{tr}\left(e^{-H}\right)}=\langle H\rangle\langle\delta H\rangle-\langle H \delta H\rangle,
$$

where in the last expression the expectation values are computed with the unperturbed density matrix. In deriving eq. (A.8), we have treated $\delta H$ as a numerical perturbation rather than as an operator. This approach is justified here because we are manipulating the operators under the trace and taking only terms which are functions of $H$ with 
only a single operator $\delta H$. Hence it is not necessary to keep track of the ordering of operators.

However, this formula assumes the perturbation of $\rho$ is small with respect to $\rho$. At this point, we have to be careful in QFT because density matrices have an infinite number of eigenvalues, which have to suffer small deviations. For example, inserting a pure particle excitation, which is well localized inside the bulk of a large region $A$, should not change very much the entropy with respect to the vacuum state. In particular, as the particle is far from the boundary $\partial A$, where most of the entanglement is produced, the entropy should be approximately the same as in the vacuum state. However $\Delta\langle H\rangle$ will measure the energy of the particle wave packet. Of course, the reason for the discrepancy between $\Delta S$ and $\Delta\langle H\rangle$ in this case is that the particle state never approaches the vacuum state while the distance $R$ between the wave packet and the boundary of the region is greater than the wavelength $\lambda$ of the wave packet. In fact, the global state with the particle excitation is always orthogonal to the global vacuum and we expect the relative entropy to increase to infinity in the limit of large $R / \lambda$, corresponding to perfect distinguishability. Further, due to the uncertainty relations the energy of the particle scales as $1 / \lambda$ and $\Delta\langle H\rangle \sim R / \lambda$.

We can formulate the following intuitive picture as to when the equality (A.6) is applicable. Near the boundary of a region, the density matrices will have a Rindler form (1.9), which suggests a thermal interpretation in the sense of Unruh [32]. In particular, there is a high temperature near the boundary and the temperature decreases with $1 / x$ as we move into the bulk of the region, where $x$ is the distance to the boundary. For a finite region of size $R$ then, there is a minimal temperature $T \sim 1 / R$ [67]. Now we want to change the state by adding some perturbation. Suppose then that we have a thermal state and mix it with a state $|E\rangle\langle E|$ of energy $E$ with some small probability $p$. In order that the change in eigenvalues is small, we must take $p \ll e^{-\beta E} / Z=p_{E}$, i.e., $p$ must be smaller than the probability with which the same state appears in the thermal ensemble. The latter is always be achieved if the change in energy is smaller than the typical average energy for the same state in the thermal bath. Hence in our original problem, we require that the energy density deposited at a location, where the local temperature is roughly $T(x)$, must be much smaller that $T^{d}$. Then the change in the entropy satisfies $\Delta S \sim \Delta E / T \ll 1$ and we are perturbing the thermal bath by our thermodynamical analogy. Otherwise, the injection of excitations in the region produces a far-from-equilibrium state.

The conclusion is that we can probe the equality (A.6) for compact regions with any state in the limit of small stress tensor expectation value. We can have small energy density perturbations inside $A$ by taking an admixture (pure or impure) of the wave packet with the vacuum. For example $|0\rangle+\epsilon|\phi\rangle$ for small $\epsilon$. In this case we can make 
the energy density of the state as low as we want without requiring the state to be of large wavelength.

\section{A.2 Strong subadditivity}

Mutual information $I(A, B)=S(A)+S(B)-S(A B)$ between two subsystems $A$ and $B$ is a measure of the information shared by them. It can be written as a particular relative entropy,

$$
I(A, B)=S\left(\rho_{A B} \mid \rho_{A} \otimes \rho_{B}\right) .
$$

Mutual information is positive and increasing with size as a consequence of the positivity and monotonicity of relative entropy. The monotonicity of the mutual information gives

$$
I(A, B C)-I(A, B)=S(A B)+S(B C)-S(A B C)-S(B) \geq 0 .
$$

Then, strong subadditivity, which is the last inequality, is implied by monotonicity of relative entropy. Note that using other properties of the entropy, one can also prove the monotonicity of relative entropy starting from strong subadditivity [27]. However, the monotonicity of relative entropy that we discuss in this paper does not reduce to strong subadditivity of entropy for different regions in space. ${ }^{23}$ Instead, if written in terms of strong subadditivity, it would involve a different kind of partition of the global Hilbert space, combined with the property that the entropy is concave.

\section{A.3 Second law of thermodynamics}

The oldest physical interpretation of the positivity of the relative entropy $S\left(\rho_{1} \mid \rho_{0}\right)$ is in terms of thermodynamics. As we described in the introduction, if $\rho_{0}$ is the equilibrium state at temperature $T$, then the relative entropy takes the form $S\left(\rho_{1} \mid \rho_{0}\right)=\left(F\left(\rho_{1}\right)-\right.$ $\left.F\left(\rho_{0}\right) / T\right)$, where $F(\rho)=\operatorname{tr}(\rho E)-T S(\rho)$ is the free energy evaluated for a general state $\rho$ but at a fixed temperature $T$. Hence, the positivity of relative entropy has the meaning that the free energy is minimal for the equilibrium state.

The thermodynamical version of this inequality is now a consequence of the second law. In general, for a system held in contact with a thermal bath at temperature $T$, the second law implies that the following the inequality holds in any process:

$$
\delta F \leq \delta W
$$

where $\delta W$ is the work done on the system. Hence it must be that for a spontaneous transformation, in which no work is done, one must have $\delta F \leq 0$. That is, the free energy must decrease as the system evolves towards equilibrium.

\footnotetext{
${ }^{23}$ The latter has been discussed previously in the AdS/CFT context by [69].
} 
The second law can be proved using properties of the relative entropy under certain assumptions for the quantum time evolution [66, 70]. We also note that relative entropy inequalities have been applied to prove the generalized second law in the context of black hole evaporation $[71,72]$.

In these proofs the second law is related to a generalized monotonicity property: The relative entropy always decreases under completely positive trace preserving (CPTP) maps between states. The CPTP maps are thought as very general class physical quantum evolutions of states [53]. For example, the evolution a subsystem which is initially decoupled from the rest, and where the global system undergoes unitary evolution, is CPTP.

The second law states that the entropy of an isolated system cannot decrease. Of course a completely isolated system in quantum mechanics evolves unitarily and the entropy does not change. We have to soften the condition of being completely isolated in order to allow for some interchange of information with the ambient space. As a model for this evolution consider the case of a quantum system with state $\rho(t)$ evolving under CPTP maps. Assume, in accordance with the idea of an "isolated" system, that the total energy $E$ is conserved. Also assume that time evolution preserves the thermal equilibrium state ${ }^{24} \rho_{T}=e^{-H / T} / \operatorname{tr}\left(e^{-H / T}\right)$ at some temperature $T$, which corresponds to the conserved energy $E, \operatorname{tr}\left(\rho_{T} H\right)=E$.

Then the relative entropy $S\left(\rho(t), \rho_{T}\right)$ is decreased by the CPTP evolution, and we have for $t_{1}<t_{2}$,

$$
F\left(\rho\left(t_{2}\right)\right)-F\left(\rho_{T}\right)<F\left(\rho\left(t_{1}\right)\right)-F\left(\rho_{T}\right),
$$

where we used that the thermal state is invariant under time evolution. Expressing this relation in terms of entropy and energy, and considering all the involved energies are the same by assumption, we have

$$
S\left(t_{2}\right)>S\left(t_{1}\right)
$$

as required by the second law of thermodynamics. Note that the difference in free energies between the state and the thermal state is positive and decreases in time. As a consequence, the state approaches the thermal equilibrium state during evolution. Eventually, if thermal equilibrium is reached, this free energy difference goes to zero.

Another case where the relative entropy allows one to prove the second law is when the totally random state $\rho_{0}=I / n$, where $n$ is the dimension of the Hilbert space, is preserved under a CPTP evolution. This state can be regarded as the microcanonical distribution. The second law follows from the fact that the relative entropy is in this

\footnotetext{
${ }^{24}$ In fact it is only necessary that there is a state such that its entropy and energies are preserved.
} 
case

$$
S\left(\rho(t) \mid \rho_{0}\right)=\log (n)-S(\rho(t)) .
$$

The increase in entropy then follows again by the decrease of relative entropy.

\section{A.4 Bekenstein bound}

The Bekenstein bound [59] is a proposal that all systems in nature should satisfy an inequality of the form

$$
S \leq 2 \pi R E,
$$

where $S$ and $E$ are the entropy and energy of a system confined to a region of size $R$. This proposed bound follows from considerations of thought experiments involving black holes. However eq. (A.15) does not involve Newton's constant and thus it should express a general property that even applies outside of the context of gravity. In particular, it should be possible to understand eq. (A.15) purely in terms of flat space physics. While this inequality appears to have a rather simple form, discussions of its possible validity, e.g., [61, 62, 73, 74], revealed a variety of subtleties in interpreting the various quantities appearing in eq. (A.15). Eventually, it was realized that a well defined version of this bound in QFT is given by the positivity of the relative entropy between two states reduced to a given region [60,61]. The connection between relative entropy and the Bekenstein bound is essentially established by eq. (1.8).

To better understand this connection between relative entropy and the Bekenstein bound, let us re-visit Bekenstein's original thought experiment [59]. Imagine that a small probe is released to fall into a large black hole, from a short distance $R$ above the horizon. ${ }^{25}$ The object then disappears behind the horizon carrying entropy $S$ and energy $E$, as measured by a local observer at the point from which it was released. The energy swallowed by the black hole as measured asymptotically is red-shifted to $E T_{\mathrm{BH}} / T_{\mathrm{rel}} \simeq 2 \pi R E T_{\mathrm{BH}}$, where $T_{\mathrm{BH}}$ and $T_{\text {rel }}$ are the Hawking temperature measured at infinity and the local temperature measure at the release radius, respectively. Hence the variation of the black hole's mass is $\delta M=2 \pi R E T_{B H}$ and the corresponding variation in the horizon entropy is given by $\delta S_{\mathrm{BH}}=\delta M / T_{\mathrm{BH}}=2 \pi R E$. Finally the generalized second law demands that the increase in the horizon entropy must at least compensate for the loss of entropy in the exterior region, i.e., $\delta S_{\mathrm{BH}} \geq S$, and hence we have arrived at the bound (A.15).

A drawback of the expression (A.15) is that the entropy (and the energy) of a finite region are not well defined quantities. In order to eliminate the ambiguities in the definition of the entropy, it was argued in [61] that the relevant quantity for

\footnotetext{
${ }^{25}$ Implicitly, we assume that the size $\lambda$ of the probe is smaller than the original distance above the horizon, i.e., $R \gtrsim \lambda$.
} 
Bekenstein's thought experiment was the difference of entropies between the state in the relevant region $V$ and the vacuum entropy in the same region $\Delta S=S\left(\rho_{V}\right)-S\left(\rho_{V}^{0}\right)$. In Bekenstein's thought experiment, $V$ is the near horizon region just outside of the black hole. In fact then, there is a large entanglement entropy, which can be seen as the entropy in the thermal atmosphere around the black hole, both for the object localized to the region outside the event horizon and for the vacuum state localized in the same region. These are the initial and final states of the process and so only the change in entropy, i.e., the difference between the two entanglement entropies, enters into the inequality. That is, we should interpret $S$ appearing on the left-hand side of eq. (A.15) as $\Delta S$, the same difference which appears on the right-hand side of eq. (1.8).

Further, the quantity $2 \pi R E$ appearing on the right-hand side of eq. (A.15) suffers from similar ambiguities. However, this product can also be given a precise meaning as $\Delta\langle H\rangle$, the difference in expectation values of the modular Hamiltonian (for $\rho_{V}^{0}$ ) between the two states [60]. To make this connection precise, we first note that in Bekenstein's thought experiment, the relevant physics for the near horizon region of a large black hole is very nearly the same as that for Rindler space. Hence, recall the modular Hamiltonian in Rindler space is given by eq. (1.9). Hence evaluating $\Delta\langle H\rangle$ between the state with Bekenstein's probe near the horizon and the vacuum state, we find

$$
\Delta\langle H\rangle=2 \pi \int_{x>0} d^{d-1} x x\left\langle T_{00}(x)\right\rangle_{\rho_{V}} \simeq 2 \pi R E .
$$

Hence $\Delta\langle H\rangle$ reproduces the expression appearing on the right-hand side of eq. (A.15) in Bekenstein's thought experiment and the inequality (A.15) found there is nothing but the inequality (1.8) expressing the positivity of the relative entropy (1.8). Of course, $\Delta\langle H\rangle$ also provides an unambiguous definition for the product of energy and size when applying the Bekenstein bound to more general systems and more general regions.

This discussion shows a well-defined version of the Bekenstein bound in QFT is given by the positivity of the relative entropy $\Delta S \leq \Delta\langle H\rangle$ between an arbitrary state and the vacuum state, both reduced to some finite region $V$ [60]. This relative entropy bound holds automatically, implying, despite the use of black holes in Bekenstein's thought experiment, that the physics behind the Bekenstein bound is simply quantum mechanics and special relativity. It also generalizes the Bekenstein bound to arbitrary regions, since the original derivation by Bekenstein is limited to Rindler space.

\section{Relative entropy kills the species problem}

Interestingly, the version of the Bekenstein bound arising from relative entropy, i.e., eq. (1.8) does not suffer from the species problem [61]. That is, considering theories with a large number of species or different quantum fields will not lead to violations 
of eq. (1.8). This is because as the number of degrees of freedom is increased, the entropy of a localized excitation can be made bigger for the same energy, but the entropy already present in the vacuum entanglement also gets larger. Since $\Delta S$ is bounded by $\Delta\langle H\rangle$, the difference in the entropies must converge to a fixed value as the number of species becomes arbitrarily large. In terms of the relative entropy, adding more species makes the distinguishability between the localized object and the localized vacuum poorer, reducing the relative entropy. However, the distinguishability is always positive, only becoming zero for the identical states. That is, an increased number of species may mean that we will be closer to saturating the bound but it can never produce violations of the inequality (1.8). The role of Hawking radiation and black hole thermal atmosphere in preserving the bound in Bekenstein's thought experiment is then information theoretical and not mechanical, in the sense that radiation pressure on the infalling object does not play a decisive role, as is sometimes considered, e.g., [73].

To see how the species problem is solved in more detail, we start by describing the way it was originally posed, i.e., let us look at a canonical case with many species. In particular, let us consider a theory consisting $\mathcal{N}$ decoupled copies of some QFT. For a moment, let us set aside the idea of bounded regions and consider global states. Let $\hat{\rho}_{0}=|0\rangle\langle 0|$ be the global vacuum for a single species, and $\hat{\rho}_{1}=|\psi\rangle\langle\psi|$ is any other orthogonal pure state (e.g., a one-particle state). We start with the global vacuum $|\Omega\rangle=|0\rangle \otimes \cdots \otimes|0\rangle$ and the corresponding density matrix

$$
\rho_{0}=|\Omega\rangle\langle\Omega|=\hat{\rho}_{0} \otimes \cdots \otimes \hat{\rho}_{0}
$$

Now we replace the vacuum by the excited state $|\psi\rangle$ in the $i$ 'th copy of the field theory, i.e., $\left|\Psi_{i}\right\rangle=|0\rangle \otimes \cdots \otimes|\psi\rangle \otimes \cdots \otimes|0\rangle$. Then the corresponding density matrix becomes

$$
\rho_{i}=\left|\Psi_{i}\right\rangle\left\langle\Psi_{i}\right|=\hat{\rho}_{0} \otimes \cdots \otimes \hat{\rho}_{1} \otimes \cdots \otimes \hat{\rho}_{0}
$$

So the states $\rho_{i}$ are pure and we also have they correspond to orthogonal vectors, $\left\langle\Psi_{i} \mid \Psi_{j}\right\rangle=0$ if $i \neq j$. Hence, the mixed density matrix obtained by combining these particle excitations for the different species as

$$
\rho_{\text {mix }}=\frac{1}{\mathcal{N}} \sum \rho_{i}=\frac{1}{\mathcal{N}} \sum\left|\Psi_{i}\right\rangle\left\langle\Psi_{i}\right|
$$

is already diagonalized in the basis of the $\left|\Psi_{i}\right\rangle$. It has $\mathcal{N}$ non-zero eigenvalues with value $1 / \mathcal{N}$. Hence it follows that $S\left(\rho_{\text {mix }}\right)=\log (\mathcal{N})$ and

$$
\Delta S_{\text {tot }}=S\left(\rho_{\text {mix }}\right)-S\left(\rho_{0}\right)=\log (\mathcal{N}) .
$$


Here $\Delta S$ increases without bound as $\mathcal{N}$ grows, while the energy in $\rho_{\text {mix }}$ is independent of $\mathcal{N}$.

Considerations of a similar nature have been used to produce contradictions with Bekenstein bound e.g., [74]. However, note that as we are considering global states here, it is natural to assume that $R \rightarrow \infty$ and so our discussion leads to no contradiction with eq. (A.15). The discussion is slightly different using the interpretation in terms of relative entropy. In this case, one may note that the global orthogonal pure states $\hat{\rho}_{0}$ and $\hat{\rho}_{1}$ within a single copy are perfectly distinguishable and hence their relative entropy is infinite. The same holds for the states in eqs. (A.18) and (A.19), e.g., $S\left(\rho_{\text {mix }} \mid \rho_{0}\right)=\infty$. What allows $\Delta S_{\text {tot }}$ in eq. (A.20) to increase without bound is the fact that $\Delta\langle H\rangle$ is already divergent. Formally, this divergence can be seen as arising in writing $|0\rangle\langle 0|$ as $\sim e^{-H}$, we introduced an infinite coefficient for the orthogonal projector $|\psi\rangle\langle\psi|$ in in the modular Hamiltonian $H$. For a more intuitive insight, let us instead consider a thermal ensemble $\rho_{T} \sim e^{-\mathcal{H} / T}$ with $H=\mathcal{H} / T$, where $\mathcal{H}$ is the usual Hamiltonian. Now, the vacuum density matrix $\rho_{0}$ can be seen as the zero temperature limit and hence given that $|\psi\rangle$ has a fixed finite energy, one finds $\Delta H \rightarrow \infty$ as $T \rightarrow 0$.

However, these are global states, and a finite size $R$ is necessary to formulate the Bekenstein bound in a sensible way. For simplicity then, let us consider the case of reduced states inside a ball $V$ of radius $R$. The reduced state of the vacuum becomes

$$
\rho_{0}=\operatorname{Tr}_{\bar{V}}[|\Omega\rangle\langle\Omega|]=\hat{\rho}_{0} \otimes \cdots \otimes \hat{\rho}_{0}
$$

where now $\hat{\rho}_{0}=\operatorname{tr}_{\bar{V}}[|0\rangle\langle 0|]$ is the 'vacuum' density matrix in each individual copy of the field theory. Note that we are introducing Tr to denote tracing in the full Hilbert space, i.e., over all copies of the field theory, and tr to denote a trace in a single copy of the field theory. Now constructing the analogous density matrices for the excited states (A.19) yields

$$
\rho_{i}=\operatorname{Tr}_{\bar{V}}\left[\left|\Psi_{i}\right\rangle\left\langle\Psi_{i}\right|\right]=\hat{\rho}_{0} \otimes \cdots \otimes \hat{\rho}_{1} \otimes \cdots \otimes \hat{\rho}_{0}
$$

where $\hat{\rho}_{1}=\operatorname{tr}_{\bar{V}}[|\psi\rangle\langle\psi|]$. Further the corresponding mixed state is

$$
\rho_{\text {mix }}=\frac{1}{\mathcal{N}} \sum \rho_{i}
$$

Now as the different copies are all decoupled, the modular Hamiltonian takes the form $H_{\text {tot }}=\sum H_{i}$ where

$$
H_{i}=\mathbf{1}_{1} \otimes \mathbf{1}_{2} \otimes \cdots \otimes H \otimes \cdots \otimes \mathbf{1}_{\mathcal{N}} .
$$

In this expression, the $H$ appearing as the $i$ 'th entry in the direct product is precisely the modular Hamiltonian for a single copy of the QFT. 
Now let consider a situation analogous to the one above, where we have a pure excitation which is as different as possible from the vacuum. For global states, distinguishability of vacuum and particle states is infinite. However, inside the sphere, this must be bounded. In order for the excited state to be as different as possible from the vacuum in the sphere, we should construct a wave packet with a very short wavelength $\lambda$ far from the spherical boundary (well inside where the effective temperature is low). Now if we specialize to the case where the QFT's under consideration are conformal field theories, the modular Hamiltonian $H$ is given by eq. (1.10) and we can make a precise statement. In particular, placing the wave packet at the center of the sphere, we find

$$
\Delta H=\pi \frac{R}{\lambda} \gg 1
$$

Certainly this result can be very large and when $\frac{R}{\lambda} \gg 1$, we approach the that situation the excited state is maximally distinguishable from $\rho_{0}$. Note however, that while it can be large, $\Delta H$ will never be divergent in the bounded region. Further, in this regime, the entropy calculation is approximately same as described for the global states above and we have

$$
\Delta\langle H\rangle-\Delta S=\pi \frac{R}{\lambda}-\log (\mathcal{N}) .
$$

As $\mathcal{N}$ increases the relative entropy decreases (the bound becomes tighter) as expected, since relative entropy always decreases under mixing [27]

$$
S\left(\sum p_{i} \rho_{i}^{(1)} \mid \sum p_{i} \rho_{i}^{(2)}\right) \leq \sum p_{i} S\left(\rho_{i}^{(1)} \mid \rho_{i}^{(2)}\right)
$$

for $p_{i}>0$ and $\sum p_{i}=1$. However, since $\Delta\langle H\rangle$ is independent of $\mathcal{N}$ and relative entropy is always positive, the $\log (\mathcal{N})$ behavior of $\Delta S$ can not subsist for a very large number of species $\mathcal{N} \gtrsim e^{R / \lambda}$. Finally eq. (1.8) must be saturated with $\Delta S=\Delta\langle H\rangle$. Clearly there must be a change in the behavior $\Delta S$ away from the simple logarithmic growth found in eq. (A.20) in the regime where $\mathcal{N} \gtrsim e^{R / \lambda}$. Intuitively, the probability of finding an excited wave packet from the $i$ 'th copy of the CFT in the vacuum density matrix (which has an effective temperature of roughly $1 / R$ at the wave packet location) is $e^{-R / \lambda} / Z$ independently of $\mathcal{N}$. For the excited state in $\rho_{\text {mix }}$, this probability becomes $\frac{1}{\mathcal{N}}+\frac{e^{-R / \lambda}}{Z}$. Hence when $\mathcal{N} \gtrsim e^{R / \lambda}$, the vacuum and the mixed state are no longer very different and we are actually in a regime where $\Delta S \simeq \Delta\langle H\rangle$.

Hence, we see the importance both of expressing the original product $2 \pi R E$ on the right-hand side of the bound (A.15) as the change in the modular 'energy' $\Delta\langle H\rangle$, and of considering the entropy difference $\Delta S$, rather than simply the entropy $S$. This last step ensures that $\Delta S$ saturates the bound in the case of large number of species. When the number of species is sufficiently large, the particle excitation whose probability is 
distributed amongst the various copies in the mixed state is hidden behind the cloud of excitations produced simply localizing the vacuum to a finite region. Hence $\rho_{\text {mix }}$ and $\rho_{0}$ are no longer easily distinguished.

In general, the transition from the form in eq. (A.26) to zero for large enough $\mathcal{N}$ will be some complicated function. However, let us determine the first nontrivial corrections for the case of small deviations from vacuum state, i.e., the opposite regime to that just analyzed above. ${ }^{26}$ Let us begin by considering the pure states (A.22). Within any individual copy of the QFT, if $\hat{\rho}_{1}$ is a small perturbation of the vacuum density matrix $\hat{\rho}_{0}$, then we will find as usual

$$
\Delta\langle H\rangle=\Delta S
$$

Of course, for the copies containing no excitations, we find simply $\Delta\langle H\rangle=\Delta S=0$. Hence for these pure states, we find $\left.\Delta\left\langle H_{\text {tot }}\right\rangle\right|_{\rho_{i}}=\left.\Delta S_{\text {tot }}\right|_{\rho_{i}}$, as expected.

Now for the mixed state (A.23), we find

$$
\begin{aligned}
\left.\Delta\left\langle H_{\text {tot }}\right\rangle\right|_{\rho_{\text {mix }}} & =\frac{1}{\mathcal{N}} \sum\left(\operatorname{Tr}\left[\rho_{i} H_{\text {tot }}\right]-\operatorname{Tr}\left[\rho_{0} H_{\text {tot }}\right]\right) \\
& =\frac{1}{\mathcal{N}} \sum\left(\operatorname{tr}\left[\hat{\rho}_{1} H\right]-\operatorname{tr}\left[\hat{\rho}_{0} H\right]\right)_{i} \\
& =\frac{1}{\mathcal{N}} \sum \Delta\langle H\rangle_{i}=\Delta\langle H\rangle
\end{aligned}
$$

where the subscript $i$ in the second and third sums indicates that the corresponding expression is evaluated only in the $i$ 'th copy of the QFT. The final $\Delta\langle H\rangle$ can be evaluated in any single copy of the field theory and so the shift in the expectation value of the modular Hamiltonian is unchanged that would be found for any of the pure states $\rho_{i}$. Similarly, following our standard reasoning, one also finds $\left.\Delta S_{\text {tot }}\right|_{\rho_{\text {mix }}}=\Delta\langle H\rangle$, as usual for small deviations from the vacuum. That is, the new mixed state saturates the inequality (1.8) with precisely the same values as the individual pure states $\rho_{i}$, to first order. That is, these first order calculations do not distinguish the pure and mixed states.

However, the mixed state should have more entropy than the pure states and so we must go to higher orders, we should see this difference. As in the holographic calculations in section 3 , going to higher orders means evaluating the change in entropy to higher orders since the linear calculations of $\Delta\left\langle H_{\text {tot }}\right\rangle$ are complete. To begin let us write the excited state within a single copy of the field theory as

$$
\hat{\rho}_{1}=\hat{\rho}_{0}+\delta \hat{\rho}=\hat{\rho}_{0}\left(\mathbf{1}+\hat{\rho}_{0}^{-1} \delta \hat{\rho}\right)
$$

\footnotetext{
${ }^{26}$ Note that the following analysis would apply for any finite region and for a tensor product of $\mathcal{N}$ copies of any QFT.
} 
Further note that since $\operatorname{tr}\left[\hat{\rho}_{1}\right]=1=\operatorname{tr}\left[\hat{\rho}_{0}\right]$, we must have $\operatorname{tr}[\delta \hat{\rho}]=0$. To introduce some more notation, let us write the $i$ 'th pure state as

$$
\rho_{i} \equiv \rho_{0}\left[\mathbf{1}+\delta \tilde{\rho}_{i}\right] \equiv e^{-H_{\text {tot }}} e^{-\widetilde{\delta H}_{i}}
$$

where

$$
\delta \tilde{\rho}_{i} \equiv \mathbf{1}_{1} \otimes \cdots \otimes \hat{\rho}_{0}^{-1} \delta \hat{\rho} \otimes \cdots \otimes \mathbf{1}_{\mathcal{N}}
$$

with $\hat{\rho}_{0}^{-1} \delta \hat{\rho}$ appearing in the $i$ 'th factor of the tensor product. The 'effective' shift in the modular Hamiltonian $\widetilde{\delta H}_{i}$ defined by eq. (A.31) is related to $\delta \tilde{\rho}_{i}$ by

$$
\widetilde{\delta H}_{i}=-\log \left(\mathbf{1}+\delta \tilde{\rho}_{i}\right)=-\delta \tilde{\rho}_{i}+\frac{1}{2} \delta \tilde{\rho}_{i}^{2}-\frac{1}{3} \delta \tilde{\rho}_{i}^{3}+\cdots
$$

Note that the definition of $\widetilde{\delta H}_{i}$ involves the product of two separate exponentials. So in general, it does not precisely match the shift $\delta H_{i}$ appearing in the conventional definition: $\rho_{i} \equiv \exp \left[-H_{\text {tot }}-\delta H_{i}\right]$ because $\delta H_{i}$ does not commute with $H_{\text {tot }}$. That is, $\widetilde{\delta H}_{i}=\delta H_{i}$ requires $\left[H_{\text {tot }}, \delta H_{i}\right]=0$.

Having established this notation, we would like to compare the shift in the entanglement entropy for the perturbed pure states (A.22) with that for the perturbed mixed state (A.23). Towards that end, it is convenient to use the Baker-Campbell-Hausdorff formula to expand the logarithm appearing in the entanglement entropy. For example, we encounter

$$
\begin{aligned}
\log \rho_{i}= & \log \left[e^{-H_{\mathrm{tot}}} e^{-\widetilde{\delta H}_{i}}\right] \\
=-H_{\mathrm{tot}} & -\widetilde{\delta H}_{i}+\frac{1}{2}\left[H_{\mathrm{tot}}, \widetilde{\delta H_{i}}\right]-\frac{1}{12}\left[H_{\mathrm{tot}},\left[H_{\mathrm{tot}}, \widetilde{\delta H}_{i}\right]\right]+\frac{1}{12}\left[\widetilde{\delta H}_{i},\left[H_{\mathrm{tot}}, \widetilde{\delta H}_{i}\right]\right] \\
& \quad-\frac{1}{24}\left[\widetilde{\delta H}_{i},\left[H_{\mathrm{tot}},\left[H_{\mathrm{tot}}, \widetilde{\delta H}_{i}\right]\right]\right]+\cdots,
\end{aligned}
$$

where the terms denoted by the ellipsis will involve four and more commutators of $H_{\text {tot }}$ and $\widetilde{\delta H}_{i}$. Note that in the present calculation, we will only concern ourselves with the terms with two or fewer $\widetilde{\delta H}_{i}$ 's, however, there are an infinite number of such contributions. However, we will only need to understand the general form of these terms for the present comparison.

Applying the above definitions, we find for the pure states

$$
\begin{aligned}
\left.\Delta S_{\mathrm{tot}}\right|_{\rho_{i}}= & -\operatorname{Tr}\left[\rho_{i} \log \rho_{i}\right]+\operatorname{Tr}\left[\rho_{0} \log \rho_{0}\right] \\
=\operatorname{Tr} & {\left[\rho_{0}\left(\widetilde{\delta H}_{i}-\frac{1}{2}\left[H_{\mathrm{tot}}, \widetilde{\delta H}_{i}\right]+\frac{1}{12}\left[H_{\mathrm{tot}},\left[H_{\mathrm{tot}}, \widetilde{\delta H}_{i}\right]\right]-\frac{1}{12}\left[\widetilde{\delta H}_{i},\left[H_{\mathrm{tot}}, \widetilde{\delta H}_{i}\right]+\cdots\right)\right]\right.} \\
& +\operatorname{Tr}\left[\rho_{0} \delta \tilde{\rho}_{i}\left(H_{\mathrm{tot}}+\widetilde{\delta H}_{i}-\frac{1}{2}\left[H_{\mathrm{tot}}, \widetilde{\delta H}_{i}\right]+\frac{1}{12}\left[H_{\mathrm{tot}},\left[H_{\mathrm{tot}}, \widetilde{\delta H}_{i}\right]\right]-\cdots\right)\right] .
\end{aligned}
$$


Again, there is an infinite number of terms for each order in $\widetilde{\delta H}_{i}$ (or $\left.\delta \tilde{\rho}_{i}\right)$ in the above expression. However, with the trace above, there is an enormous simplification with

$$
\operatorname{Tr}\left[\rho_{0}\left[H_{\mathrm{tot}}, Z\right]\right]=\operatorname{Tr}\left[\rho_{0} H_{\mathrm{tot}} Z\right]-\operatorname{Tr}\left[H_{\mathrm{tot}} \rho_{0} Z\right]=0
$$

for any matrix $Z$ since $H_{\text {tot }}=-\log \rho_{0}$ commutes with $\rho_{0}$. Taking this simplification into account, there are only two potential contributions at linear order,

$$
\begin{aligned}
\left.\Delta S_{\text {tot }}\right|_{\rho_{i}, \text { linear }} & =-\operatorname{Tr}\left[\rho_{0} \delta \tilde{\rho}_{i}\right]+\operatorname{Tr}\left[\rho_{0} \delta \tilde{\rho}_{i} H_{\text {tot }}\right] \\
& =-\operatorname{tr}[\delta \hat{\rho}]+\operatorname{tr}[\delta \hat{\rho} H]=\Delta\langle H\rangle,
\end{aligned}
$$

where the reduction between the first and second lines relies on the tensor product structure of the various matrices and $\operatorname{tr}[\delta \hat{\rho}]=0$. Of course, this shift in the entropy at linear order agrees with $\left.\Delta\left\langle H_{\text {tot }}\right\rangle\right|_{\rho_{i}}=\Delta\langle H\rangle$, as in our previous discussion above. Now the quadratic contributions take the form

$$
\begin{aligned}
\left.\Delta S_{\text {tot }}\right|_{\rho_{i}, \text { quad }}=\operatorname{Tr} & {\left[\rho_{0}\left(\frac{1}{2} \delta \tilde{\rho}_{i}^{2}-\frac{1}{12}\left[\delta \tilde{\rho}_{i},\left[H_{\text {tot }}, \delta \tilde{\rho}_{i}\right]\right]+\frac{1}{24}\left[\delta \tilde{\rho}_{i},\left[H_{\text {tot }},\left[H_{\text {tot }}, \delta \tilde{\rho}_{i}\right]\right]\right]+\cdots\right)\right] } \\
& -\operatorname{Tr}\left[\rho_{0} \delta \tilde{\rho}_{i}\left(\delta \tilde{\rho}_{i}-\frac{1}{2}\left[H_{\text {tot }}, \delta \tilde{\rho}_{i}\right]+\frac{1}{12}\left[H_{\text {tot }},\left[H_{\text {tot }}, \delta \tilde{\rho}_{i}\right]\right]-\cdots\right)\right](\text { A. } .38)
\end{aligned}
$$

Again there is an infinite number of terms in both lines above. We will not attempt to simplify eq. (A.38) for the states $\rho_{i}$ further. Rather we now turn to considering the mixed state (A.23).

Hence, for the mixed state (A.23), we can define

$$
\rho_{\text {mix }}=\rho_{0}\left[\mathbf{1}+\frac{1}{\mathcal{N}} \sum \delta \tilde{\rho}_{i}\right] \equiv e^{-H_{\text {tot }}} e^{-\widetilde{\delta H}_{\text {mix }}}
$$

where $\delta \tilde{\rho}_{i}$ is defined in eq. (A.32). Further, the effective shift in the modular Hamiltonian $\widetilde{\delta H}_{\text {mix }}$ defined above can be written as

$$
\widetilde{\delta H}_{\text {mix }}=-\log \left(\mathbf{1}+\frac{1}{\mathcal{N}} \sum \delta \tilde{\rho}_{i}\right)=-\frac{1}{\mathcal{N}} \sum \delta \tilde{\rho}_{i}+\frac{1}{2} \frac{1}{\mathcal{N}^{2}} \sum_{i, j} \delta \tilde{\rho}_{i} \delta \tilde{\rho}_{j}+\cdots
$$

Note that $\widetilde{\delta H}_{\text {mix }} \neq \frac{1}{\mathcal{N}} \sum \widetilde{\delta H}_{i}$ since the latter sum would not contain all of the crossterms appearing in eq. (A.40).

Now it is a straightforward exercise to verify using the above expressions that to linear order, we have: $\left.\Delta S_{\text {tot }}\right|_{\rho_{\text {mix }} \text {, linear }}=\Delta\langle H\rangle=\left.\left\langle\Delta H_{\text {tot }}\right\rangle\right|_{\rho_{\text {mix }}}$. Turning then to the 
quadratic contributions, we have

$$
\begin{aligned}
\left.\Delta S_{\text {tot }}\right|_{\rho_{\text {mix }}, \text { quad }}= & \frac{1}{\mathcal{N}^{2}} \sum_{i, j} \operatorname{Tr}\left[\rho_{0}\left(\frac{1}{2} \delta \tilde{\rho}_{i} \delta \tilde{\rho}_{j}-\frac{1}{12}\left[\delta \tilde{\rho}_{i},\left[H_{\text {tot }}, \delta \tilde{\rho}_{j}\right]\right]+\frac{1}{24}\left[\delta \tilde{\rho}_{i},\left[H_{\text {tot }},\left[H_{\text {tot }}, \delta \tilde{\rho}_{j}\right]\right]\right]+\cdots\right)\right] \\
& -\frac{1}{\mathcal{N}^{2}} \sum_{i, j} \operatorname{Tr}\left[\rho_{0} \delta \tilde{\rho}_{i}\left(\delta \tilde{\rho}_{j}-\frac{1}{2}\left[H_{\text {tot }}, \delta \tilde{\rho}_{j}\right]+\frac{1}{12}\left[H_{\text {tot }},\left[H_{\text {tot }}, \delta \tilde{\rho}_{j}\right]\right]-\cdots\right)\right] \quad(\text { A.41) } \\
= & \frac{1}{\mathcal{N}^{2}} \sum_{i, j} \operatorname{Tr}\left[\rho_{0}\left(\frac{1}{2} \delta \tilde{\rho}_{i} \delta \tilde{\rho}_{j}-\frac{1}{12}\left[\delta \tilde{\rho}_{i},\left[H_{j}, \delta \tilde{\rho}_{j}\right]\right]+\frac{1}{24}\left[\delta \tilde{\rho}_{i},\left[H_{j},\left[H_{j}, \delta \tilde{\rho}_{j}\right]\right]\right]+\cdots\right)\right] \\
& -\frac{1}{\mathcal{N}^{2}} \sum_{i, j} \operatorname{Tr}\left[\rho_{0} \delta \tilde{\rho}_{i}\left(\delta \tilde{\rho}_{j}-\frac{1}{2}\left[H_{j}, \delta \tilde{\rho}_{j}\right]+\frac{1}{12}\left[H_{j},\left[H_{j}, \delta \tilde{\rho}_{j}\right]\right]-\cdots\right)\right] .
\end{aligned}
$$

In the second equality, we have emphasized that because of the tensor product structure of $\delta \tilde{\rho}_{i}$ given in eq. (A.32), only the corresponding terms of $H_{\text {tot }}=\sum H_{i}$ contribute in the commutators. Further combining this structure with $\operatorname{tr}[\hat{\rho}]=0$, we have that all of the terms with $i \neq j$ above will vanish. Hence all of the double sums can be reduced as follows, e.g.,

$$
\begin{aligned}
\frac{1}{\mathcal{N}^{2}} \sum_{i, j} \operatorname{Tr}\left[\rho_{0} \delta \tilde{\rho}_{i}\left[H_{j}, \cdots\left[H_{j}, \delta \tilde{\rho}_{j}\right]\right]\right] & =\frac{1}{\mathcal{N}^{2}} \sum_{i} \operatorname{Tr}\left[\rho_{0} \delta \tilde{\rho}_{i}\left[H_{i}, \cdots\left[H_{i}, \delta \tilde{\rho}_{i}\right]\right]\right] \\
& =\frac{1}{\mathcal{N}} \operatorname{Tr}\left[\rho_{0} \delta \tilde{\rho}_{1}\left[H_{1}, \cdots\left[H_{1}, \delta \tilde{\rho}_{1}\right]\right]\right]
\end{aligned}
$$

where we have eliminated the sum in the last expression and chosen $i=1$ as a representative value, by using the fact that all of the terms in the previous diagonal sum are identical. Hence the quadratic shift in the entropy simplifies to

$$
\begin{array}{r}
\left.\Delta S_{\text {tot }}\right|_{\rho_{\text {mix }}, \text { quad }}=\frac{1}{\mathcal{N}} \operatorname{Tr}\left[\rho_{0}\left(\frac{1}{2} \delta \tilde{\rho}_{1}^{2}-\frac{1}{12}\left[\delta \tilde{\rho}_{1},\left[H_{\text {tot }}, \delta \tilde{\rho}_{1}\right]\right]+\frac{1}{24}\left[\delta \tilde{\rho}_{1},\left[H_{\text {tot }},\left[H_{\text {tot }}, \delta \tilde{\rho}_{1}\right]\right]\right]+\cdots\right)\right] \\
-\frac{1}{\mathcal{N}} \operatorname{Tr}\left[\rho_{0} \delta \tilde{\rho}_{1}\left(\delta \tilde{\rho}_{1}-\frac{1}{2}\left[H_{\text {tot }}, \delta \tilde{\rho}_{1}\right]+\frac{1}{12}\left[H_{\text {tot }},\left[H_{\text {tot }}, \delta \tilde{\rho}_{1}\right]\right]-\cdots\right)\right](\text { A. } 43)
\end{array}
$$

Here again, we have an infinite number of contributions above but comparing this result with eq. (A.38), it is clear that we have $\left.\Delta S_{\text {tot }}\right|_{\rho_{\text {mix }}, \text { quad }}=\left.\frac{1}{\mathcal{N}} \Delta S_{\text {tot }}\right|_{\rho_{i}, \text { quad }}$. That is, at quadratic order, we have

$$
\left.\left(\Delta\left\langle H_{\text {tot }}\right\rangle-\Delta S_{\text {tot }}\right)\right|_{\rho_{\text {mix }}}=\left.\frac{1}{\mathcal{N}}\left(\Delta\left\langle H_{\text {tot }}\right\rangle-\Delta S_{\text {tot }}\right)\right|_{\rho_{i}}+O\left(\delta \tilde{\rho}_{i}^{3}\right) .
$$

Note that the above analysis did not reveal much about the structure of the quadratic contributions and so we did not actually establish that the shifts in the entropy in eqs. (A.38) and (A.43) are negative. However, the latter is easily shown by 
introducing the standard representation of the logarithm in terms of the resolvent, as follows

$$
\log (\rho+\delta \rho)=-\int_{0}^{\infty} d \beta\left[\frac{1}{\rho+\delta \rho+\beta}-\frac{1}{\beta+1}\right] .
$$

The advantage of this representation is that even when $\rho$ and $\delta \rho$ do not commute, it is straightforward to expand the above expression for small perturbations with

$$
\frac{1}{\rho+\delta \rho+\beta}=\frac{1}{\rho+\beta}-\frac{1}{\rho+\beta} \delta \rho \frac{1}{\rho+\beta}+\frac{1}{\rho+\beta} \delta \rho \frac{1}{\rho+\beta} \delta \rho \frac{1}{\rho+\beta}+\cdots
$$

Now for any of the pure global states where the excitations appear in one copy of the QFT, it is straightforward to show

$$
\begin{aligned}
\left.\Delta S_{\mathrm{tot}}\right|_{\rho_{i}} & =-\operatorname{Tr}\left[\rho_{i} \log \rho_{i}\right]+\operatorname{Tr}\left[\rho_{0} \log \rho_{0}\right] \\
& =-\operatorname{tr}\left[\hat{\rho}_{i} \log \hat{\rho}_{i}\right]+\operatorname{Tr}\left[\hat{\rho}_{0} \log \hat{\rho}_{0}\right] \\
& =-\operatorname{tr}\left[\left(\hat{\rho}_{0}+\delta \hat{\rho}\right) \log \left(\hat{\rho}_{0}+\delta \hat{\rho}\right)\right]+\operatorname{Tr}\left[\hat{\rho}_{0} \log \hat{\rho}_{0}\right]
\end{aligned}
$$

That is, as before, the simple tensor product structure of $\rho_{i}$ and $\rho_{0}$ allows us to reduce the calculation of $\Delta S_{\text {tot }}$ to the single copy of the QFT carrying the excitation. Now we can apply eqs. (A.45) and (A.46) to this expression. Examining the terms linear in $\delta \hat{\rho}$, one again finds $\Delta S_{\text {tot }}=\Delta\left\langle H_{\text {tot }}\right\rangle$. Hence to leading order, we recover the equality already found twice above. Now also including the second order terms, we find

$$
\left.\left(\Delta\left\langle H_{\text {tot }}\right\rangle-\Delta S_{\text {tot }}\right)\right|_{\rho_{i}}=\int_{0}^{\infty} d \beta \beta \operatorname{tr}\left(\frac{1}{\hat{\rho}_{0}+\beta} \delta \hat{\rho} \frac{1}{\hat{\rho}_{0}+\beta} \delta \hat{\rho} \frac{1}{\hat{\rho}_{0}+\beta}\right)+\cdots
$$

Note the second order term above is explicitly positive since the matrix $\left(\hat{\rho}_{0}+\beta\right)^{-1}$ in the center of the integrand is positive definite. Further, this expression now captures all of the second order terms and so as required the relative entropy is positive. Of course, given the result in eq. (A.44), the same positivity applies for the mixed state.

As a final comment, let us note that Bekenstein's thought experiment involves a dynamical process and the exchange of entropy and energy between two systems. Interpreting the Bekenstein bound in terms of relative entropy, the same reasoning can also be applied in flat space and for any region, in particular without referring to black holes. The flat space experiment would involve an excitation with a modular energy difference $\Delta\langle H\rangle$ with respect to the vacuum in a region $V$. Under some evolution this modular energy (the Rindler energy in Bekenstein's experiment) is assumed to be at the same time conserved but passed to a thermal reservoir i.e., being converted into 'heat' in the thermodynamical language (represented by the black hole in Bekenstein's 
thought experiment). ${ }^{27}$ This gives $\Delta S_{\text {res }}=\Delta\langle H\rangle$ because for the reservoir, with a large number of degree of freedom, we are always in the small deviation scenario (note the temperature here is $T=1$ ). The increase of the entropy under this evolution requires $\Delta S_{\text {res }}-\Delta S=\Delta\langle H\rangle-\Delta S \geq 0$. In fact, as shown in section A.3, positivity of relative entropy can always be interpreted in this way as a consequence of a second law for specific time evolutions which are CPTP but nonunitary in the region. A simple example for the present case is given by an evolution which adds identical and independent field species and mixes the state in such larger Hilbert space, as described above in this section. This process may represent for our purposes, the evolution of the initial system which is finally absorbed by the reservoir. Implicitly, the above discussion shows that this 'evolution' preserves the value of $\Delta\langle H\rangle$. Also in the limit of a large number of species, we should get $\Delta S_{\text {res }}=\Delta\langle H\rangle$. Here $\Delta S_{\text {res }}$ is the variation of the entropy of the bath due to presence of the probe, which is now distributed among a large number of field species. Hence, the relative entropy bound can also be considered a cons equence of a second law under a CPTP evolution, in analogy with the derivation of the Bekenstein bound using the generalized second law.

\section{References}

[1] See, for example:

M. Levin and X.-G. Wen, "Detecting Topological Order in a Ground State Wave Function," Phys. Rev. Lett. 96, 110405 (2006) [arXiv:cond-mat/0510613];

A. Kitaev and J. Preskill, "Topological entanglement entropy," Phys. Rev. Lett. 96, 110404 (2006) [arXiv:hep-th/0510092];

A. Hamma, R. Ionicioiu and P. Zanardi, "Ground state entanglement and geometric entropy in the Kitaev's model," Phys. Lett. A 337, 22 (2005)

[arXiv:quant-ph/0406202].

[2] See, for example:

P. Calabrese and J. L. Cardy, "Entanglement entropy and quantum field theory," J. Stat. Mech. 0406, P002 (2004) [arXiv:hep-th/0405152];

P. Calabrese and J. L. Cardy, "Entanglement entropy and quantum field theory: A non-technical introduction," Int. J. Quant. Inf. 4, 429 (2006) [arXiv:quant-ph/0505193].

[3] See, for example:

I. R. Klebanov, D. Kutasov and A. Murugan, "Entanglement as a Probe of Confinement," Nucl. Phys. B 796, 274 (2008) [arXiv:0709.2140 [hep-th]];

\footnotetext{
${ }^{27}$ Note that in Bekenstein's experiment, the initial Rindler energy is conserved along ordinary time evolution in the form of ordinary energy because it is proportional to the energy as meassured by asymptotic observers.
} 
T. Nishioka and T. Takayanagi, "AdS Bubbles, Entropy and Closed String Tachyons," JHEP 0701, 090 (2007) [hep-th/0611035];

P. V. Buividovich and M. I. Polikarpov, "Numerical study of entanglement entropy in SU(2) lattice gauge theory," Nucl. Phys. B 802, 458 (2008) [arXiv:0802.4247 [hep-lat]]; Y. Nakagawa, A. Nakamura, S. Motoki and V. I. Zakharov, "Quantum entanglement in SU(3) lattice Yang-Mills theory at zero and finite temperatures," PoS LATTICE 2010, 281 (2010) [arXiv:1104.1011 [hep-lat]].

[4] H. Casini, M. Huerta, "A Finite entanglement entropy and the c-theorem," Phys. Lett. B600, 142-150 (2004). [hep-th/0405111].

[5] V. Balasubramanian, M. B. McDermott and M. Van Raamsdonk, "Momentum-space entanglement and renormalization in quantum field theory," Phys. Rev. D 86, 045014 (2012) [arXiv:1108.3568 [hep-th]].

[6] H. Casini and M. Huerta, "On the RG running of the entanglement entropy of a circle," Phys. Rev. D 85, 125016 (2012) [arXiv:1202.5650 [hep-th]].

[7] R. C. Myers and A. Sinha, "Seeing a c-theorem with holography," Phys. Rev. D 82, 046006 (2010) [arXiv:1006.1263 [hep-th]];

R. C. Myers and A. Sinha, "Holographic c-theorems in arbitrary dimensions," JHEP 1101, 125 (2011) [arXiv:1011.5819 [hep-th]].

[8] R. D. Sorkin, "On the Entropy of the Vacuum Outside a Horizon," in General Relativity and Gravitation, Volume 1, B. Bertotti, F. de Felice and A. Pascolini, ed., p. 734 (1983);

L. Bombelli, R. K. Koul, J. Lee and R. D. Sorkin, "A Quantum Source of Entropy for Black Holes," Phys. Rev. D 34, 373 (1986);

M. Srednicki, "Entropy and area," Phys. Rev. Lett. 71, 666 (1993) [hep-th/9303048]; V. P. Frolov and I. Novikov, "Dynamical origin of the entropy of a black hole," Phys. Rev. D 48, 4545 (1993) [gr-qc/9309001].

[9] L. Susskind and J. Uglum, "Black hole entropy in canonical quantum gravity and superstring theory," Phys. Rev. D 50, 2700 (1994) [hep-th/9401070].

[10] S. N. Solodukhin, "Entanglement entropy of black holes," Living Rev. Rel. 14, 8 (2011) [arXiv:1104.3712 [hep-th]].

[11] A. Almheiri, D. Marolf, J. Polchinski and J. Sully, "Black Holes: Complementarity or Firewalls?," JHEP 1302, 062 (2013) [arXiv:1207.3123 [hep-th]].

[12] S. D. Mathur, "The Information paradox: A Pedagogical introduction," Class. Quant. Grav. 26, 224001 (2009) [arXiv:0909.1038 [hep-th]].

[13] S. L. Braunstein, S. Pirandola and K. Zyczkowski, "Entangled black holes as ciphers of hidden information," Physical Review Letters 110, 101301 (2013) [arXiv:0907.1190 [quant-ph]]. 
[14] M. Van Raamsdonk, "Comments on quantum gravity and entanglement," arXiv:0907.2939 [hep-th];

M. Van Raamsdonk, "Building up spacetime with quantum entanglement," Gen. Rel. Grav. 42, 2323 (2010) [arXiv:1005.3035 [hep-th]].

[15] V. E. Hubeny and M. Rangamani, "Causal Holographic Information," arXiv:1204.1698 [hep-th];

B. Czech, J. L. Karczmarek, F. Nogueira and M. Van Raamsdonk, "The Gravity Dual of a Density Matrix," arXiv:1204.1330 [hep-th].

[16] E. Bianchi and R. C. Myers, "On the Architecture of Spacetime Geometry," arXiv:1212.5183 [hep-th].

[17] S. Ryu and T. Takayanagi, "Holographic derivation of entanglement entropy from AdS/CFT," Phys. Rev. Lett. 96, 181602 (2006) [arXiv:hep-th/0603001];

S. Ryu and T. Takayanagi, "Aspects of holographic entanglement entropy," JHEP 0608, 045 (2006) [arXiv:hep-th/0605073];

T. Nishioka, S. Ryu and T. Takayanagi, "Holographic Entanglement Entropy: An Overview," J. Phys. A 42, 504008 (2009) [arXiv:0905.0932 [hep-th]];

T. Takayanagi, "Entanglement Entropy from a Holographic Viewpoint," arXiv:1204.2450 [gr-qc].

[18] M. Headrick, "Entanglement Renyi entropies in holographic theories," Phys. Rev. D 82, 126010 (2010) [arXiv:1006.0047 [hep-th]].

[19] L. Y. Hung, R. C. Myers and M. Smolkin, "On Holographic Entanglement Entropy and Higher Curvature Gravity," JHEP 1104, 025 (2011) [arXiv:1101.5813 [hep-th]].

[20] H. Casini, M. Huerta and R. C. Myers, "Towards a derivation of holographic entanglement entropy," JHEP 1105, 036 (2011) [arXiv:1102.0440 [hep-th]].

[21] A. Lewkowycz and J. Maldacena, "Generalized gravitational entropy," arXiv:1304.4926 [hep-th].

[22] A. Rényi, "On measures of information and entropy," in Proceedings of the 4th Berkeley Symposium on Mathematics, Statistics and Probability, 1, 547 (U. of California Press, Berkeley, CA, 1961);

A. Rényi, "On the foundations of information theory," Rev. Int. Stat. Inst. 33 (1965) 1.

[23] For example, see:

K. Zyczkowski, "Renyi extrapolation of Shannon entropy," Open Syst. Inf. Dyn. 10, 297 (2003) [arXiv:quant-ph/0305062];

C. Beck and F. Schlögl, "Thermodynamics of chaotic systems", (Cambridge University Press, Cambridge, 1993).

[24] P. Calabrese1 and A. Lefevre, "Entanglement spectrum in one-dimensional systems," Phys. Rev. A 78, 032329 (2008) [arXiv:0806.3059 [cond-mat.str-el]]. 
[25] T. Faulkner, "The Entanglement Renyi Entropies of Disjoint Intervals in AdS/CFT," arXiv:1303.7221 [hep-th];

T. Hartman, "Entanglement Entropy at Large Central Charge," arXiv:1303.6955 [hep-th].

[26] L.-Y. Hung, R. C. Myers, M. Smolkin and A. Yale, "Holographic Calculations of Renyi Entropy," JHEP 1112, 047 (2011) [arXiv:1110.1084 [hep-th]].

[27] A. Wehrl, "General properties of entropy", Rev. Mod. Phys. 50, 221 (1978).

[28] V. Vedral, "The role of relative entropy in quantum information theory", Rev. Mod. Phys. 74, 197 (2002).

[29] R. Haag, "Local quantum physics: Fields, particles, algebras", Berlin, Germany: Springer (1992) (Texts and monographs in physics).

[30] See, for example:

H. Li and F. D. M. Haldane, "Entanglement Spectrum as a Generalization of Entanglement Entropy: Identification of Topological Order in Non-Abelian Fractional Quantum Hall Effect States," Phys. Rev. Lett. 101, 010504 (2008) [arXiv:0805.0332 [cond-mat.mes-hall]];

P. Calabrese and A. Lefevre, "Entanglement spectrum in one-dimensional systems," Phys. Rev. A 78, 032329 (2008);

A. M. Turner, F. Pollmann and E. Berg, "Topological Phases of One-Dimensional Fermions: An Entanglement Point of View," Phys. Rev. B 83, 075102 (2011) [arXiv:1008.4346 [cond-mat.str-el]];

L. Fidkowski, "Entanglement spectrum of topological insulators and superconductors," Phys. Rev. Lett. 104, 130502 (2010) [arXiv:0909.2654 [cond-mat.str-el]];

H. Yao and X.-L. Qi, "Entanglement entropy and entanglement spectrum of the Kitaev model," Phys. Rev. Lett. 105, 080501 (2010) [arXiv:1001.1165 [cond-mat.str-el]].

[31] J. J. Bisognano and E. H. Wichmann, "On The Duality Condition For Quantum Fields," J. Math. Phys. 17, 303 (1976);

J. J. Bisognano and E. H. Wichmann, "On The Duality Condition For A Hermitian Scalar Field," J. Math. Phys. 16, 985 (1975).

[32] W. G. Unruh, "Notes on black hole evaporation," Phys. Rev. D 14, 870 (1976).

[33] P. D. Hislop and R. Longo, "Modular Structure Of The Local Algebras Associated With The Free Massless Scalar Field Theory," Commun. Math. Phys. 84, 71 (1982);

[34] H. J. Borchers and J. Yngvason, "Modular groups of quantum fields in thermal states," J. Math. Phys. 40, 601 (1999) [math-ph/9805013].

[35] See, for example:

S. de Haro, S. N. Solodukhin and K. Skenderis, "Holographic reconstruction of 
spacetime and renormalization in the AdS/CFT correspondence," Commun. Math. Phys. 217, 595 (2001) [arXiv:hep-th/0002230];

K. Skenderis, "Lecture notes on holographic renormalization," Class. Quant. Grav. 19, 5849 (2002) [arXiv:hep-th/0209067].

[36] R. C. Myers, "Stress tensors and Casimir energies in the AdS/CFT correspondence," Phys. Rev. D 60, 046002 (1999) [arXiv:hep-th/9903203].

[37] W. Fischler, A. Kundu and S. Kundu, "Holographic Mutual Information at Finite Temperature," arXiv:1212.4764 [hep-th].

[38] J. Bhattacharya, M. Nozaki, T. Takayanagi and T. Ugajin, "Thermodynamical Property of Entanglement Entropy for Excited States," arXiv:1212.1164 [hep-th].

[39] V. E. Hubeny, M. Rangamani and T. Takayanagi, "A Covariant holographic entanglement entropy proposal," JHEP 0707, 062 (2007) [arXiv:0705.0016 [hep-th]].

[40] R. C. Myers, M. F. Paulos and A. Sinha, "Holographic Hydrodynamics with a Chemical Potential," JHEP 0906, 006 (2009) [arXiv:0903.2834 [hep-th]].

[41] C. Fefferman and C. R. Graham, "Conformal Invariants," in Elie Cartan et les Mathématiques d'aujourd hui (Astérisque, 1985) 95;

C. Fefferman and C. R. Graham, "The Ambient Metric," arXiv:0710.0919 [math.DG].

[42] S. 'i. Nojiri and S. D. Odintsov, "On the conformal anomaly from higher derivative gravity in AdS/CFT correspondence," Int. J. Mod. Phys. A 15, 413 (2000) [hep-th/9903033].

[43] O. Aharony, S. S. Gubser, J. M. Maldacena, H. Ooguri and Y. Oz, "Large N field theories, string theory and gravity," Phys. Rept. 323, 183 (2000) [arXiv:hep-th/9905111].

[44] A. Schwimmer and S. Theisen, "Entanglement Entropy, Trace Anomalies and Holography," Nucl. Phys. B 801, 1 (2008) [arXiv:0802.1017 [hep-th]].

[45] L.-Y. Hung, R. C. Myers and M. Smolkin, "Some Calculable Contributions to Holographic Entanglement Entropy," JHEP 1108, 039 (2011) [arXiv:1105.6055 [hep-th]].

[46] M. Banados, C. Teitelboim and J. Zanelli, "The Black hole in three-dimensional space-time," Phys. Rev. Lett. 69, 1849 (1992) [arXiv:hep-th/9204099].

[47] S. W. Hawking and D. N. Page, "Thermodynamics of Black Holes in anti-De Sitter Space," Commun. Math. Phys. 87, 577 (1983);

E. Witten, "Anti-de Sitter space, thermal phase transition, and confinement in gauge theories," Adv. Theor. Math. Phys. 2, 505 (1998) [hep-th/9803131].

[48] V. Korepin, "Universality of entropy scaling in 1D gap-less models," Phys. Rev. Lett. 92, 096402 (2004) [arXiv:cond-mat/0311056]. 
[49] C. Holzhey, F. Larsen and F. Wilczek, "Geometric and renormalized entropy in conformal field theory," Nucl. Phys. B 424, 443 (1994) [hep-th/9403108].

[50] See, for example:

P. Di Francesco, P. Mathieu and D. Senechal, "Conformal field theory," New York, USA: Springer (1997) $890 \mathrm{p}$

[51] J. M. Maldacena and A. Strominger, "AdS(3) black holes and a stringy exclusion principle," JHEP 9812, 005 (1998) [hep-th/9804085].

[52] See, for example:

S. S. Gubser, "Curvature singularities: The Good, the bad, and the naked," Adv. Theor. Math. Phys. 4, 679 (2000) [hep-th/0002160];

R. C. Myers and O. Tafjord, "Superstars and giant gravitons," JHEP 0111, 009 (2001) [hep-th/0109127].

[53] M. A. Nielsen, I. L. Chuang, Quantum Computation and quantum Information, Cambridge Univ. Press., Cambridge (2000).

[54] H. Casini, M. Huerta and R. C. Myers, "Mutual information and a c-theorem for $d=3$, , in preparation.

[55] See for example H. Halvorson, "Reeh-Schlieder defeats Newton-Wigner: On alternative localization schemes in relativistic quantum field theory," Phil. Sci. 68, 111 (2001) [quant-ph/0007060].

[56] L. Y. Hung, R. C. Myers and M. Smolkin, "Twist operators in higher dimensions," in preparation.

[57] E. Bianchi and R. C. Myers, "On the Architecture of Spacetime Geometry," arXiv:1212.5183 [hep-th].

[58] H. Liu and A. A. Tseytlin, "On four point functions in the CFT / AdS correspondence," Phys. Rev. D 59, 086002 (1999) [hep-th/9807097];

E. D'Hoker and D. Z. Freedman, "Supersymmetric gauge theories and the AdS/CFT correspondence," hep-th/0201253.

[59] J. D. Bekenstein, "A Universal Upper Bound on the Entropy to Energy Ratio for Bounded Systems," Phys. Rev. D 23, 287 (1981);

J. D. Bekenstein, "Generalized second law of thermodynamics in black hole physics," Phys. Rev. D 9, 3292 (1974).

[60] H. Casini, "Relative entropy and the Bekenstein bound," Class. Quant. Grav. 25, 205021 (2008) [arXiv:0804.2182 [hep-th]].

[61] D. Marolf, D. Minic and S. F. Ross, "Notes on space-time thermodynamics and the observer dependence of entropy," Phys. Rev. D 69, 064006 (2004) [hep-th/0310022]; D. Marolf, "A Few words on entropy, thermodynamics, and horizons," hep-th/0410168. 
[62] R. Bousso, "Light sheets and Bekenstein's bound," Phys. Rev. Lett. 90, 121302 (2003) [hep-th/0210295].

[63] E. Bianchi, "Horizon entanglement entropy and universality of the graviton coupling," arXiv:1211.0522 [gr-qc].

[64] M. Nozaki, T. Numasawa, A. Prudenziati and T. Takayanagi, "Dynamics of Entanglement Entropy from Einstein Equation," arXiv:1304.7100 [hep-th];

M. Nozaki, T. Numasawa and T. Takayanagi, "Holographic Local Quenches and Entanglement Density," arXiv:1302.5703 [hep-th].

[65] D. Allahbakhshi, M. Alishahiha and A. Naseh, "Entanglement Thermodynamics," arXiv:1305.2728 [hep-th];

G. Wong, I. Klich, L. A. P. Zayas and D. Vaman, "Entanglement Temperature and Entanglement Entropy of Excited States," arXiv:1305.3291 [hep-th].

[66] V. Vedral, Introduction to quantum information science, Oxford University Press, New York (2006).

[67] P. Martinetti and C. Rovelli, "Diamonds's temperature: Unruh effect for bounded trajectories and thermal time hypothesis," Class. Quant. Grav. 20, 4919 (2003) [gr-qc/0212074].

[68] V. Balasubramanian, P. Kraus and A. E. Lawrence, "Bulk versus boundary dynamics in anti-de Sitter space-time," Phys. Rev. D 59, 046003 (1999) [hep-th/9805171].

[69] M. Headrick and T. Takayanagi, "A Holographic proof of the strong subadditivity of entanglement entropy," Phys. Rev. D 76, 106013 (2007) [arXiv:0704.3719 [hep-th]];

A. C. Wall, "Maximin Surfaces, and the Strong Subadditivity of the Covariant Holographic Entanglement Entropy," arXiv:1211.3494 [hep-th].

[70] T. Sagawa, "Second law-like inequalities with quantum relative entropy: An introduction", arXiv:1202.0983 [cond-mat.stat-mech]

[71] R.D. Sorkin, "Toward an Explanation of Entropy Increase in the Presence of Quantum Black Holes", Phys. Rev. Lett. 56, 1885 (1986); R. D. Sorkin, "The statistical mechanics of black hole thermodynamics", arXiv:gr-qc/9705006.

[72] A. C. Wall, "A proof of the generalized second law for rapidly changing fields and arbitrary horizon slices," Phys. Rev. D 85, 104049 (2012) [arXiv:1105.3445 [gr-qc]]; A. C. Wall, "A Proof of the generalized second law for rapidly-evolving Rindler horizons," Phys. Rev. D 82, 124019 (2010) [arXiv:1007.1493 [gr-qc]].

[73] M. A. Pelath and R. M. Wald, "Comment on entropy bounds and the generalized second law," Phys. Rev. D 60, 104009 (1999) [gr-qc/9901032];

D. Marolf and R. D. Sorkin, "On the status of highly entropic objects," Phys. Rev. D 69, 024014 (2004) [hep-th/0309218]. 
[74] D. N. Page, "Comment On A Universal Upper Bound On The Entropy To Energy Ratio For Bounded Systems," Phys. Rev. D 26, 947 (1982);

W. G. Unruh and R. M. Wald, "Acceleration Radiation and Generalized Second Law of Thermodynamics," Phys. Rev. D 25, 942 (1982). 